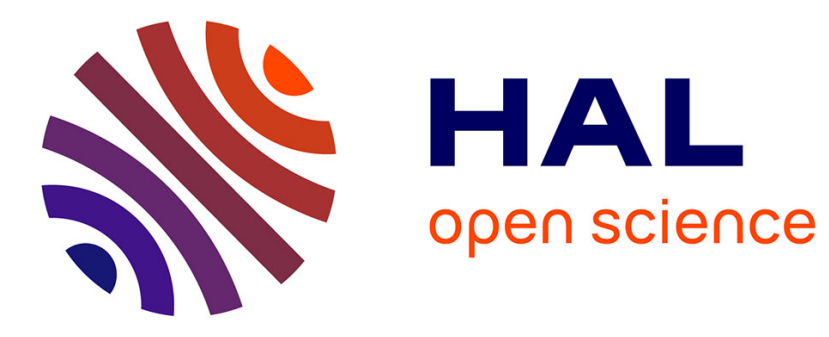

\title{
The acetylene ground state saga
}

Michel Herman

\section{To cite this version:}

Michel Herman. The acetylene ground state saga. Molecular Physics, 2008, 105 (17-18), pp.2217-2241. 10.1080/00268970701518103 . hal-00513123

\section{HAL Id: hal-00513123 \\ https://hal.science/hal-00513123}

Submitted on 1 Sep 2010

HAL is a multi-disciplinary open access archive for the deposit and dissemination of scientific research documents, whether they are published or not. The documents may come from teaching and research institutions in France or abroad, or from public or private research centers.
L'archive ouverte pluridisciplinaire HAL, est destinée au dépôt et à la diffusion de documents scientifiques de niveau recherche, publiés ou non, émanant des établissements d'enseignement et de recherche français ou étrangers, des laboratoires publics ou privés. 


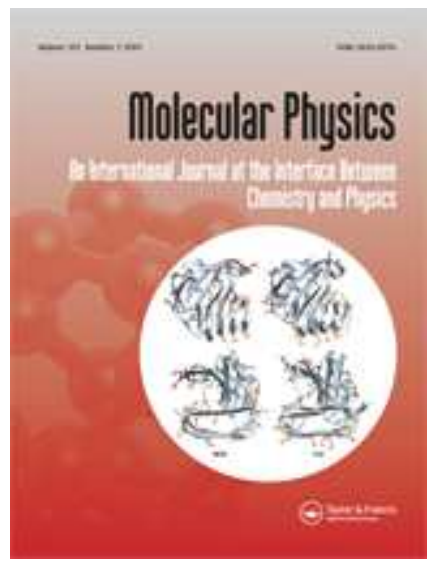

\section{The acetylene ground state saga}

\begin{tabular}{|r|l|}
\hline Journal: & Molecular Physics \\
\hline Manuscript ID: & TMPH-2007-0155 \\
\hline Manuscript Type: & Invited Article \\
\hline Date Submitted by the \\
Author: & $30-$ May-2007 \\
\hline Complete List of Authors: & $\begin{array}{l}\text { Herman, Michel; Université Libre de Bruxelles, Chimie quantique et } \\
\text { Photophysique }\end{array}$ \\
\hline Keywords: & $\begin{array}{l}\text { acetylene, global picture, high resolution spectroscopy, } \\
\text { instrumental developments, intramolecular dynamics }\end{array}$ \\
\hline & \\
\hline
\end{tabular}

\section{今scholaroNE \\ Manuscript Central}


11 July 2010

\author{
Invited Manuscript
}

The acetylene ground state saga

\author{
Michel HERMAN \\ Service de Chimie quantique et Photophysique, CP 160/09 \\ Université libre de Bruxelles (U.L.B.) \\ Ave. Roosevelt, 50. \\ B-1050 \\ Brussels \\ Belgium \\ mherman@ulb.ac.be
}

Pages : 57

Figures : 32 (including 2 colour figures)

Tables : 3

This $m s$ was written using "century schoolbook" and, whenever necessary, "symbol" fonts. A careful distinction was made between italic vee's and Greek nu's both in the text and in formulas, that is important to respect. It will only be visible if using "century schoolbook" as the major font to visualize and print the text. 


\begin{abstract}
The evolution of the high-resolution spectroscopic investigation of the vibration-rotation energy states of acetylene in its ground electronic state is presented, with focus on advances co-authored by the ULB group. The emergence of a global picture accounting for all available spectroscopic fingerprints, at their full accuracy, is highlighted. Contribution of this research to various topics is illustrated, including instrumental developments, local mode trends, quantum vs. classical correspondence, energy $v s$. time approaches and nucleation processes.
\end{abstract}




\section{Introduction}

As reported by Miller in a book dedicated to acetylene [1], Edmund Davy told the 1836 meeting of the British Association at Bristol that [2]

“in attempting to procure potassium by strongly heating a mixture of calcined tartar and charcoal in a large iron bottle, I obtained a black substance which readily decomposed water and yielded a gas which proved to be a new compound of carbon and hydrogen. This gas burns in air with a bright flame, denser and of greater splendour than even olefiant gas...In contact with chlorine, instant explosion takes place, accompanied by a large red flame and the deposition of much carbon...The new gas requires for its complete combustion 2,5 volumes of oxygen gas, which are converted into two volumes of carbonic acid and water, which are the only products of its combustion... It is admirably adapted for the purposes of artificial light if it can be procured at a cheap rate”

Edmund Davy identified this new gas as a "new (bi)carburet of hydrogen". It was rediscovered in 1860 by French chemist Marcellin Berthelot, who coined the name "acétylène."

As stated in an encyclopaedia: Acetylene (systematic name: ethyne) is the simplest alkyne hydrocarbon, consisting of two hydrogen atoms and two carbon atoms. Because it contains a triple bond, acetylene is an 
unsaturated chemical compound. The carbon-carbon triple bond leaves the carbon atoms with two sp hybrid orbitals for sigma bonding, placing all four atoms in the same straight line, with $\mathrm{CCH}$ bond angles of $180^{\circ}$.

Acetylene indeed provides the simplest example of carbon-carbon triple bond and, as such, is referenced for its prototype geometrical structure in textbooks, with $\mathrm{CH}$ and $\mathrm{CC}$ bonds claimed to be 120 and 106 pm long. The latest equilibrium $\left(r_{\mathrm{e}}\right)$ and ground $\left(r_{0}\right)$ internuclear distances in acetylene, from [3], are, in pm:

$$
\begin{array}{ll}
r_{e}(C H)=106.138(35) ; & r_{e}(C C)=120.292(13) \\
r_{0}(C H)=105.756(52) ; & r_{0}(C C)=120.830(20)
\end{array}(\mathrm{pm})
$$

As such, they provide the most accurate values today available for the basic $\mathrm{CC}$ triple bond and related $\mathrm{CH}$ distances. Interesting enough, these bond lengths have not been optimally determined yet [4]. In addition, the linear geometry refers to the ground electronic state and not to the first and some additional excited electronic states.

The importance of acetylene in the "real world" dealt with in encyclopaedias arises mainly from its application in synthetic organic chemistry. Numerous prototype reaction mechanisms are well known and of daily use to academic and industrial chemists.

Even in such "ordinary" chemistry, however, the detailed reaction path may involve geometrical isomers of acetylene, such as vinylidene, a 
still evanescent species apparently resisting definite experimental evidence. Furthermore, acetylene is present on Earth and in various other planetary and also in stellar atmospheres, as well as in the interstellar medium. As such, it is also a key species in astrochemistry. However, related reaction mechanisms can often hardly be unravelled by textbook knowledge in organic chemistry given the most specific experimental conditions ruling astrochemistry. Acetylene chemistry is thus far from mastered. Figure 1 presents previously unpublished spectra from the Atmospheric Chemistry Experiment (ACE) mission demonstrating the presence of acetylene in the Earth atmosphere.

\section{Insert figure 1}

Pure acetylene is a colourless, highly flammable gas with an agreeable ethereal (ether-like) odour but the odour of the commercial purity grade is distinctively garlic-like.

Reference to acetylene as a single species is misleading since for the symmetric isotopologues, including ${ }^{12} \mathrm{C}_{2} \mathrm{H}_{2}$, there are two nuclear spin isomers, ortho and para that seem not to interconvert easily, if they can ever be forced to do so one day. One should therefore rather mention two independent, coexisting acetylene molecules, each long lived and with close but distinct thermodynamical properties, possibly specific chemistry and, who knows, different smells? The lack of the expected 1:3, para:ortho 
intensity alternation in the $v_{3}$ fundamental vibration-rotation band in ${ }^{12} \mathrm{C}_{2} \mathrm{H}_{2}$ observed in the spectrum of figure 2 , at specific $J$-values is typically the observation that "spin-diggers" are searching for. It turns out, however, that this peculiar observation is rather to be attributed to a succession of emission-absorption schemes, thus not requiring any spin conversion process, as correctly interpreted at the time.

\section{Insert figure 2}

Because, acetylene is important and stable, it is a very well known chemical object, with numerous well documented properties in organic and physical chemistry. Because acetylene is light and simple, it is an ideal target to produce high quality spectroscopic data and elaborate quantum models. Furthermore, with 7 vibrational degrees of freedom including two doubly degenerate ones, acetylene supports complex intramolecular mechanisms of direct relevance to the understanding of larger species. Acetylene thus appears as a prototype vehicle to unravel macroscopic properties at the microscopic level, i.e. to melt its $\mathrm{Dr}$ Jekyll and Mr Hyde natures that we just illustrated.

Indeed, acetylene became over the years a privileged ground to build theoretical concepts and develop very high-performance 
instrumental approaches, resulting in significant advances. At the same time, the ever increasing amount and quality of data available kept stimulating ever more powerful developments in a sort of catalytic process, also feeding each time a giant virtual database. The set of high resolution spectroscopy (HiReS) fingerprints on ground state acetylene presently available in the literature is probably one of the largest in all spectroscopy, amounting to several ten of thousands vibration-rotation line energies and very many intensities and broadening coefficients, and covering a very extended excitation range. This data set is of primary importance to a number of topics more traditionally connected to HiReS, including space observation and metrology.

Ground state (GS) acetylene thus generated strong emulation, fruitful collaboration and competition, and became over the years one of the favourite target species of a number of research groups all over the world, such as those headed by, in alphabetic order, Profs/Drs G. Blanquet (FUNDP, Namur), A. Campargue (UJF Grenoble, France), F.F. Crim (U Madison, USA), G. Di Lonardo and L. Fusina (Università di Bologna, Italy), R.W. Field (MIT, USA), L. Halonen (U Helsinki, Finland), W.J. Lafferty (NIST, USA), I.M. Mills (Reading, UK), B.J. Orr (McQuarrie, Sydney/Australia), V. Perevalov (Tomsk, USSR), J. Plivà (Penn state U., USA), K.N. Rao (Ohio State U., USA) and our group in Brussels, in particular. Several hundreds of papers were published down the years, including a number of reviews [8, 3, 9], giving birth to the "acetylene saga" 
as the present paper is entitled. The reader is referred to these reviews for a more exhaustive survey of the literature. In the present paper, only some areas will be highlighted, from selected references corresponding to matters in which the Brussels group has been more active. Our input was actually initiated by the late Dr. Alec Douglas, from NRCC, Ottawa, who provided in the mid-seventies Prof. Reg. Colin from ULB with original spectroscopic plates recorded by Mr. Franz Alberti in the UV and far UV on the high-performance Ottawa vacuum spectrograph. These data, analyzed in collaboration with Prof. Ingvar Kopp, at the time at the Physics Institute in Stockholm, brought information on Rydberg states and Renner-Teller effects in various acetylene isotopologues [10, 11]. Later, new plates reached Brussels through the same connection, which were this time of primary interest to Dr. Jim Watson, from Ottawa. He was the one to unravel from these spectroscopic data several remaining issues around the very peculiar $\tilde{A}-\tilde{X}$ electronic transition [12-14], later extended to additional isotopologues [15-19]. This study indirectly highlighted perturbations in the bending vibrational ladder of states in GS acetylene, isotopologue-specific [20-23]. These features in turn motivated to a very large extent our present activity.

The present contribution thus focuses on the ULB contribution to the acetylene saga, to respond precisely to the invitation. It is split into two main sections, concerning vibrational clustering -section 2- and the global approach -section 3-, each with several subsections. Various notations and 
conventions are recalled in subsections $2 \mathrm{~A}$ and $3 \mathrm{~A}$. Final words are provided in section 4. Various isotopologues will be considered in this review, with the term isotopologue referring to a molecular entity that differs only in isotopic composition, such as ${ }^{12} \mathrm{C}_{2} \mathrm{H}_{2}$ and ${ }^{12} \mathrm{C}_{2} \mathrm{D}_{2}$. It is interesting to notice that isotopomers also exist in acetylene, i.e. isomers having the same number of each isotopic atom but differing in their positions, such as $\mathrm{H}^{12} \mathrm{C}^{13} \mathrm{CD}$ and $\mathrm{D}^{12} \mathrm{C}^{13} \mathrm{CH}$.

\section{Vibrational clustering}

\section{A. Normal modes, notations and conventions}

Acetylene possesses 7 vibrational degrees of freedom giving rise to 5 normal modes of vibration represented in figure 3 , for ${ }^{12} \mathrm{C}_{2} \mathrm{H}_{2}$. The conventional labelling is thus $v_{1}$ and $v_{3}$ for the symmetric and asymmetric $\mathrm{CH}$ stretches, respectively, $v_{2}$ for the CC stretching vibration, and $v_{4}$ (trans) and $v_{5}$ (cis) for the degenerate bends. Their symmetry is indicated in figure 3 , with the $u / g$ label only valid for centro-symmetric isotopologues. The same numbering is used for all isotopologues in the literaturee.

Insert figure 3 
In the text, we shall label the vibrational states, using

$$
\left(v_{1} v_{2} v_{3} v_{4} v_{5}\right) \text { or }\left(v_{1} v_{2} v_{3} v_{4} v_{5}, l_{4} l_{5}\right) \text { or }\left(v_{1} v_{2} v_{3} v_{4}^{l_{4}} v_{5}^{l_{5}}\right) \text { or }\left(v_{1} v_{2} v_{3} v_{4} v_{5}, k\right)
$$

with $v_{\mathrm{i}}$ the vibrational quantum number for mode $i, l_{b}$ the bending vibrational angular momentum quantum number and $k=l_{4}+l_{5}$. On some occasions, commas will separate the $v_{\mathrm{i}}$ 's in the labels. These different notations are those in the various literature references from which the figures selected for the present review originate. We shall on the other hand strictly follow here the convention

$$
K \equiv k=l_{4}+l_{5} \geq 0 \quad \text { and } \quad l_{4} \geq 0 \quad \text { if } \quad k=0
$$

Transitions will be labelled using various notations, as in the literature. We shall sometimes detail the upper and then lower vibrational quantum numbers of the states involved, such as, e.g. (01011)-(00000) or $\left(0101^{1} 1^{-1}\right)-\left(0000^{0} 0^{0}\right)$. We shall also use $v_{2}+v_{4}+v_{5}$-GS, with GS for ground state, or simply $v_{2}+v_{4}+v_{5}$ whenever the lower state is the ground state. Thus, as an example, the band $v_{5}-v_{4}$ refers to the transition between the $\left(0001^{1} 0^{0}\right)$ lower and $\left(0000^{0} 1^{1}\right)$ upper states.

The vibrational frequencies in figure 3 correspond to $\tilde{\omega}_{i}^{0}$ values as 
determined from the following expansion:

$$
\begin{aligned}
& G^{0}(v, l)=\sum_{i=1,5} \tilde{\omega}_{i}^{0} v_{i}+\sum_{i \leq j} x_{i j}^{0} v_{i} v_{j}+\sum_{b \leq b^{\prime}=4,5} g_{l_{b} l_{b^{\prime}}} l_{b} l_{b^{\prime}}+\sum_{i \leq j \leq m} y_{i j m}^{0} v_{i} v_{j} v_{m}+\sum_{i, b \leq b^{\prime}} y_{i l_{b} l_{b^{\prime}}}^{0} v_{i} l_{b} l_{b^{\prime}}+ \\
& \sum_{i \leq j \leq m \leq n} z_{i j m n} v_{i} v_{j} v_{m} v_{n}+\sum_{i \leq j, b \leq b^{\prime}} z_{i j l_{b} l_{b}}, v_{i} v_{j} l_{b} l_{b^{\prime}}+\sum_{b \leq b^{\prime} \leq b^{\prime \prime} \leq b^{\prime \prime}} z_{l_{b} b_{b} l_{b^{\prime}, l_{b}}} l_{b} l_{b^{\prime}}, l_{b^{\prime \prime}} l_{b^{\prime \prime}}
\end{aligned}
$$

Most of the terms in such expressions are not relevant for the remainder of this paper. They are all defined in [28] and in the literature referred to in that paper. Note that the $g_{l_{b} b_{b}}$ constants in Eq. (2.3), also used in our initial reports (see [12-14]), strictly correspond to $x_{l_{t} l^{\prime}}$ in [28]. As a rule, only the information on the theoretical models relevant to the discussion will be provided in the text and the reader will be referred to the literature for the full description of the vibration-rotation Hamiltonian [8]. The values for the additional vibrational constants appearing in Eq.(2.3) are listed in the references mentioned in Table 1 , some of which are gathered in [3].

We need to mention the vibrational $l$-type resonance in this introductory subsection, given by the following matrix element

$$
\left\langle l_{4} l_{5}|\hat{H}| l_{4} \mp 2, l_{5} \pm 2\right\rangle=1 / 4 r_{45}\left[\left(v_{4} \pm l_{4}\right)\left(v_{4} \mp l_{4}+2\right)\left(v_{5} \mp l_{5}\right)\left(v_{5} \pm l_{5}+2\right)\right]^{1 / 2}(2
$$

As an example, the combined role of the $g_{45}$ and $\mathbf{r}_{45}$ constants, 
respectively defined in eqs. (2.3) and (2.4) is demonstrated in figure 4.

\section{Insert figure 4}

As far as conventions are concerned, it is also worth pointing out that anharmonic resonances will be referred to in the text using the corresponding coupling scheme, $\mathrm{ij} / \mathrm{kl}$ or $\mathrm{i} / \mathrm{jkl}$ or $\mathrm{ii} / \mathrm{jj}$, related to the coupling parameters $K_{\mathrm{ij} / \mathrm{kl}}$ or $K_{\mathrm{i} j \mathrm{kl}}$ or $K_{\mathrm{ii} / \mathrm{j} j}$, meaning that the interacting vibrational states are such that $\Delta v_{i}=\Delta v_{j}= \pm 1$ and, simultaneously, $\Delta v_{k}=\Delta v_{l}=\mp 1$; or $\Delta v_{i}= \pm 1$ and $\Delta v_{j}=\Delta v_{k}=\Delta v_{l}=\mp 1$; or $\Delta v_{i}= \pm 2$ and $\Delta v_{j}=\mp 2$, respectively The anharmonic resonances relevant for acetylene include e.g. 14/35, 3/245 and $44 / 55$, as discussed in the forthcoming subsections.

As an example, the matrix element related to the first of these couplings is

$\left\langle v_{1}, v_{2}, v_{3}, v_{4}^{l_{4}}, v_{5}^{l_{5}}|\tilde{H}| v_{1}, v_{2}-1, v_{3}+1,\left(v_{4}-1\right)^{l_{4} \pm 1},\left(v_{5}-1\right)^{l_{5} \mp 1}\right\rangle=-1 / 8 K_{3 / 245}\left[\left(v_{3}+1\right) v_{2}\left(v_{4} \mp l_{4}\right)\left(v_{5} \pm l_{5}\right)\right]^{1 / 2}$

with the content of the related coupling constants unravelled e.g. in [29]. In agreement with a previous statement, the other relevant resonance matrix elements as well as higher order terms and additional constants required in the model are not further defined here and can be found e.g. in [28] and in the literature referred to in that paper. All constants and spectroscopic term values in the text will be given in $\mathrm{cm}^{-1}$, independently of the label used. 
The 5 normal modes of vibration, accounting for vibrational frequencies and anharmonicities, generate numerous states with increasing vibrational excitation. The integrated number of states in ${ }^{12} \mathrm{C}_{2} \mathrm{H}_{2}$ is shown in figure 5 (top), counting each substate with $|k| \neq 0$ for 2 in the sum. A seventh order polynomial expansion was adjusted to the top curve in figure 5, whose coefficients are listed in the figure. The resulting vibrational density can be easily inferred from this expression. It is extrapolated to higher energy and presented in figure 5 (bottom). The energy origin is set at the bottom of the potential hypersurface in figure 5 (top) and at the ground vibrational state in figure 5 (bottom), the difference between the two origins corresponding to the zero point energy (ZPE $5672 \mathrm{~cm}^{-1}$ in $\left.{ }^{12} \mathrm{C}_{2} \mathrm{H}_{2}\right)$. Some oscillation, amounting to a few tens of states, was demonstrated by Zhilinskii to occur in the integrated number of states [30]. It will be further referred to, later in the text.

\section{Insert figure 5}

\section{B. The stretching states}

The $\mathrm{CH}$ harmonic stretching frequencies in the various isotopologues scale in such a way that $\tilde{\omega}_{1}^{0}>\tilde{\omega}_{3}^{0}$, with the first, symmetric $\mathrm{CH}$ stretch frequency gaining importance from the contribution of the $\mathrm{CC}$ stretch force constant $\left(f_{22}\right)$, in a simple valence force field approximation $\left(f_{12}\right.$ 
$=0$ ). The corresponding pure $\mathrm{CH}$ stretch states can be labelled either using the normal mode picture, as defined in eq.(2.1), or using the local mode notation

$$
[m, n]_{ \pm}=1 / \sqrt{2}\left[\left|\Psi_{m}\right\rangle\left|\Psi_{n}\right\rangle \pm\left|\Psi_{n}\right\rangle\left|\Psi_{m}\right\rangle\right]
$$

with $m, n$ the number of quanta in each $\mathrm{CH}$ bond (see e.g. [31]). The local mode character of the $\mathrm{CH}$ bond is known to increase upon vibrational excitation and is often used to label the $\mathrm{CH}$ stretching states at higher energy. It leads to interesting behaviour and was exploited in the asymmetric ${ }^{12} \mathrm{C}_{2} \mathrm{HD}$ species to proceed to bond selected photodissociation [32].

The intensity of vibrational $\mathrm{CH}$ overtone transitions, $n v_{s}$, thus between ground (GS) and $v_{\mathrm{s}}=n$ states, have their transition moment given by the following matrix element

$$
\left|\left\langle\Psi_{v_{s}=n}^{v i b}\left|\mu_{\alpha}\right| \Psi_{G S}^{v i b}\right\rangle\right|^{2}=\left|R_{n v_{s}}\right|^{2}
$$

in which the induced electric dipole moment operator can be developed as a function of the undimensional normal vibrational coordinates $q_{\mathrm{s}}$ 


$$
\mu_{\alpha}=\mu_{\alpha}^{e}+\sum_{s}\left(\frac{\partial \mu_{\alpha}}{\partial q_{s}}\right)_{e} q_{s}+1 / 2 \sum_{s \leq s^{\prime}}\left(\frac{\partial^{2} \mu_{\alpha}}{\partial q_{s} \partial q_{s^{\prime}}}\right)_{e} q_{s} q_{s^{\prime}}+\ldots
$$

Thus, the well known pure vibrational matrix elements, see e.g. Table 7 in [8], lead to the $\left|\Delta v_{s}\right|=n$ selection rule, with the relative intensity in an overtone series controlled by the following matrix element and coefficient, squared:

$$
\left(\frac{\partial^{n} \mu_{\alpha}}{\partial q_{s}^{n}}\right)_{e}\left\langle\Psi_{v_{s}=n}^{v i b}\left|q_{s}^{n}\right| \Psi_{G S}^{v i b}\right\rangle
$$

Experimentally searching for successive overtone transitions therefore requires more and more instrumental sensitivity since the electric dipole numerical factor $\left(\frac{\partial^{n} \mu_{\alpha}}{\partial q_{s}^{n}}\right)_{e}$ decreases with $n$. As shown in figure 6 (top) for ${ }^{12} \mathrm{C}_{2} \mathrm{HD}$, this decrease is over one order of magnitude for each additional quantum in the overtone series, here for $\mathrm{CH}$ excitation.

\section{Insert figure 6}

In the transition moment one needs accounting for electric anharmonicity, as just performed, but one can also account for the 
mechanical anharmonicity. Each wavefunction can be developed into a weighted sum of harmonic contributions $\left(\Psi_{v_{s}}^{0}\right)$

$$
\Psi_{v_{s}=n}^{v i b}=\sum_{m} c_{m} \Psi_{v_{s}=m}^{0}
$$

As a result, various contributions can be distinguished in the transition moment, depending on the order $n$ of the electric dipole moment derivative contribution, as defined in Eq.(2.9). These contributions were unravelled by Liévin for ${ }^{12} \mathrm{C}_{2} \mathrm{HD}$ stretching overtones, matching ab initio results and experimental transition moments. The role and sign of the contribution of each order $m$ to two ( $n=3$ and 4$)$ of the observed overtone transitions is shown in figure 6 (bottom). The amount and signs of the various contributions appear to be different for different overtones bands (here $3 v_{1}$ and $4 v_{1}$ ) and actually, as shown in the referenced paper, also between $\mathrm{CH}$ and $\mathrm{CD}$ excitation bands.

The intensity information in figure 6 is extracted from spectra recorded at high resolution at ULB using Fourier transform spectroscopy (FTS). Vibration-rotation bands were observed up to the visible range, hence the denomination of "coloured" vibrations. The quality of the data is illustrated in figure 7 , which shows absorption to the $4^{\text {th }}$ overtone of the $\mathrm{CH}$ excitation $\left(5 \mathrm{CH}\right.$ ) in ${ }^{12} \mathrm{C}_{2} \mathrm{H}_{2}$. For this recording, acetylene pressure was 250 mbar, absorption pathlength was $49.2 \mathrm{~m}$ using a White type multipass 
cell, and 6900 scans recorded during some 96 hours were co-added.

\section{Insert figure 7}

Laser based experiments allow sensitivity to be boosted (see e.g. figure 68 (p.254) in [8]). The highest energy $\mathrm{CH}$ overtone transition reported in the literature lies in the blue range, close to $24000 \mathrm{~cm}^{-1}$ ! This $7^{\text {th }}$ overtone $(n=$ 8) was recorded using intracavity optoacoustic laser spectroscopy [36] and, more recently by the Grenoble group using the newly developed "cavity enhanced spectroscopy" [37]. Numerous other acetylene spectra demonstrating even higher sensitivity were recorded, as further discussed in section $2 \mathrm{D}$.

As a final word to this subsection, one can focus on the so-called $x-K$ relations, here applied to the $\mathrm{CH}$ stretchings. According to the local mode picture, the various basic vibrational parameters associated to the $\mathrm{CH}$ stretchings in the symmetric isotopologues are related through these $x-K$ relations and thus expected to fulfil the following approximate rule (see e.g. [38]):

$$
x_{11}=x_{33}=1 / 4 x_{13}=1 / 4 K_{11 / 33}
$$

with the 11/33 anharmonic resonance connecting $\mathrm{CH}$ stretching states, those with $\Delta v_{1}=-\Delta v_{3}= \pm 2$. Acetylene data allow this relation to be 
successfully checked, as demonstrated in table 1 . These relations are actually also well fulfilled in ${ }^{12} \mathrm{C}^{13} \mathrm{CH}_{2}$ [39], but not in ${ }^{12} \mathrm{C}_{2} \mathrm{HD}$ [27] because of the decoupling of the $\mathrm{CH}$ and $\mathrm{CD}$ vibrations. As a result of the 11/33 resonance, the zero order wavefunctions are mixed up and severe labelling problems may occur. These are often encountered in spectroscopy and will be highlighted on several other occasions in this review. It should be noticed, however, that mixings between $\mathrm{CH}$ wavefunctions due to $11 / 33$ are very limited, if not virtually non-existent in the case of ${ }^{12} \mathrm{C}_{2} \mathrm{D}_{2}$, due to the large offset between the $v_{1}$ and $v_{3}$ fundamental frequencies (see figure 2).

\section{Insert table 1}

\section{The bending states}

The normal mode bending motions can be understood as symmetrically and antisymmetrically coordinated variations of the two HCC angles. A look at the numbers in figure 3 shows that the two related harmonic frequencies, $\tilde{\omega}_{4}^{0}$ and $\tilde{\omega}_{5}^{0}$ are almost degenerate in ${ }^{12} \mathrm{C}_{2} \mathrm{D}_{2}$, as further discussed below, while they are far apart in ${ }^{12} \mathrm{C}_{2} \mathrm{HD}$. In the latter case $v_{4}$ and $v_{5}$ actually appear to be almost localised CCD and CCH bends, respectively. As a rule the trans-bending frequency is lower than the cisbending one. A closer look at the anharmonicity corrections shows that the series of trans- and cis-bending states do cross at high excitation, since $x_{44}$ $(>0)$ and $x_{55}(<0)$ have opposite signs (see [40] for the latest values in 
${ }^{12} \mathrm{C}_{2} \mathrm{H}_{2}$ ). This crossing occurs around $v_{\mathrm{b}}=23$ in ${ }^{12} \mathrm{C}_{2} \mathrm{H}_{2}$. Since a Darling Dennison type resonance (44/55) occurs among the bends, with related matrix elements accounting for $l$ values, the mixing is maximal at that stage and the labelling most problematic. It would be interesting to figure out the structural evolution along this series of bending overtones. This problem could possibly be related to the acetylene-vinylidene isomerization process already mentioned in the introduction. Along this line of research, the stimulated emission pumping (SEP) and dispersed laser induced fluorescence (DLIF) experimental techniques used by Field (MIT) are most efficient (e.g. [41]). Both use a two-step absorption scheme, going through the first excited electronic states as an intermediate. Thanks to the trans-geometry of this $\tilde{A}$ state and the role of Franck Condon factors, transitions towards highly excited bent states in the ground electronic state are favoured and observed up to high excitation, as will be exemplified in the next subsection. These investigations, however, did apparently not bring definite information on the isomerization process, yet.

The two bend frequencies are quite close and the related $v_{5}-v_{4}$ difference band was recorded in the far-infrared range using FTS by Johns (NRC/Ottawa) and analysed in Bologna and Brussels for ${ }^{12} \mathrm{C}_{2} \mathrm{H}_{2}[22,42,40]$ and for ${ }^{12} \mathrm{C}_{2} \mathrm{D}_{2}$ [23]. This closeness was examined by Zhilinskii and Gaspard in terms of the related vibrational density and dynamics, respectively [43]. The equilibrium vibrational frequencies in three 
symmetric isotopologues, reproduced from this reference, are provided in Table 2, including the stretching vibrations for completeness. This Table also lists the associated periods, calculated according to the relation

$$
T_{i}=\frac{2 \pi \hbar}{\omega_{i}}
$$

with $\hbar=5308.84 \mathrm{fs} \mathrm{cm}^{-1}$. The analysis demonstrates that the bends, with the slowest periodic orbits, have a major "bottleneck" contribution to the vibrational dynamics in acetylene. In ${ }^{12} \mathrm{C}_{2} \mathrm{D}_{2}$, in particular, the two bends are separated by less than $30 \mathrm{~cm}^{-1}$ and recurrence times are $T=65,130$ and $190 \mathrm{fs}$, with

$$
T \approx n_{4} T_{4} \approx n_{5} T_{5} \text { and }\left(n_{4}, n_{5}\right)=(1,1) ;(2,2) ;(3,3)
$$

\section{Insert Table 2}

The major role of the bends in the internal vibrational dynamics was demonstrated by Gaspard using vibrograms as presented in figure 8 for ${ }^{12} \mathrm{C}_{2} \mathrm{D}_{2}$. In these graphs, the energy (abscise), defined from the bottom of the potential well, runs from -4000 to $+9000 \mathrm{~cm}^{-1}$, from left to right. The integrated number of states is Fourier transformed over wide but restricted energy windows, defining the time scale (see [43] for further details). Translated to the energy picture, these time recurrences mean 
that all bend states, with their numerous $l$-components, accumulate in the integrated number of states around multiples of the mean bend frequency, i.e. about $500 \mathrm{~cm}^{-1}$ in ${ }^{12} \mathrm{C}_{2} \mathrm{D}_{2}$. This phenomenon actually leads to a slight variation of the number of states at related regular energy spacing in the integrated count compared to the regular fitted curve and, hence to small oscillations in this quantity, as already mentioned and presented in figure 8. In addition, because of this accidental near-degeneracy between the bends, the Darling Dennison mixing is very efficient in ${ }^{12} \mathrm{C}_{2} \mathrm{D}_{2}$ right from the start of the bending vibrational ladder [23].

Insert figure 8

D. The stretch-bend states

In addition to the 11/33 and 44/55 Darling Dennison resonances, several other anharmonic coupling schemes occur in acetylene. Resonances can be spotted from spectra using the concept of so-called bright and dark states, or rather bright and dark transitions leading to intensity borrowing. It is demonstrated in the sequence of schemes gathered in Eq. (2.14) and in figure 9. All labels are explained in this figure. 


$$
\begin{aligned}
& \left|\left\langle\Psi_{B}^{0}|\mu| G S\right\rangle\right|^{2} \neq 0 \\
& \left|\left\langle\Psi_{D}^{0}|\mu| G S\right\rangle\right|^{2}=0
\end{aligned} \rightarrow \begin{aligned}
& \left|\left\langle\Psi_{+} \equiv\left(a \Psi_{B}^{0}+b \Psi_{D}^{0}\right)|\mu| G S\right\rangle\right|^{2}=\begin{array}{l}
a^{2}\left|\left\langle\Psi_{B}^{0}|\mu| G S\right\rangle\right|^{2} \\
\left|\left\langle\Psi_{-} \equiv\left(b \Psi_{B}^{0}-a \Psi_{D}^{0}\right)|\mu| G S\right\rangle\right|^{2}
\end{array} b^{2}\left|\left\langle\Psi_{B}^{0}|\mu| G S\right\rangle\right|^{2}
\end{aligned}
$$

Insert figure 9

Thus, intensity borrowing occurs, more or less efficiently depending on the coupling and relative zero order energy schemes. This is illustrated in figure 10 , with the $1 / 255$ resonance in ${ }^{12} \mathrm{C}_{2} \mathrm{HD}$. All pairs of states fulfilling the $1 / 255$ coupling schemes interact, with intensity borrowing from the zero order bright to dark transitions. The relevant pairs of states can actually be systematically predicted from the full set of energies, as shown in figure 11 . In this figure, the energy difference $(\Delta \mathrm{E})$ between pairs of states interacting through the selected resonance, here $1 / 255$, is plotted as a function of the state energy. Only one of the two interacting states is identified on the figure, for clarity, using $v_{1} v_{2} v_{3} v_{4} v_{5}$. All those pairs with values of $\Delta \mathrm{E}$ small enough and with one of the corresponding transitions bright enough, can be expected to lead to intensity borrowing, as was exemplified in figure 10, in a selected energy range. 
FT spectra, recorded over very broad ranges, such as those displayed in figure 10, played a crucial role in highlighting anharmonic resonances in acetylene. Additional resonances are usually accounted for in the final vibrational fits, to improve the final residuals, provided they do not affect the polyad or cluster structure that will be defined in section 3. Most of the acetylene FT spectra were recorded at ULB or in Bologna. The "intracavity laser absorption spectroscopy (ICLAS)" data from Grenoble (e.g. [44-50], recently based on VeCSEL lasers [50], as well as other laser-based methods such as the optoacoustic technique (e.g. [51, 52]), NIR diode laser investigations (e.g. [53]), and Raman-type investigations (e.g. [54, 55]) also provided a great deal of information on the stretch-bend states up to high vibrational excitation.

In addition to relative intensities, absolute intensity measurements provide even more refined insight into intramolecular coupling schemes, as demonstrated in [56]. Furthermore, absolute intensities are of course closely associated to the quantitative exploitation of spectral fingerprints in the atmospheric and astrophysical contexts (e.g. [40]). There again FT data proved to be most relevant, not only in the lower infrared region, but also in the overtone range (e.g. [57]). The comparison with the FT data in the paper just referred to stimulated the development at ULB of so-called 2T-FT-ICLAS experiments [58]. This technique was recently demonstrated to provide accuracy better than $10 \%$ on absolute intensity measurements 
in the overtone range [59]. Figure 12 highlights the power of the 2T-FTICLAS instrumental combination, thus merging the sensitivity of intracavity absorption in a $\mathrm{Ti}$ :Sa laser and the broad spectral coverage of high resolution FTS. The efficiency of the set-up, first developed at low spectral resolution in Madrid [60], was significantly increased by designing and building a home made Ti:Sa laser for this specific experiment. In ICLAS, one injects the pump laser into the Ti:Sa cavity for a definite time, defining the generation time, $t_{g}$, during which the intracavity absorption occurs. The longer $t_{g}$, the longer the effective absorption path $l_{\text {eff }}$ in the cavity, with

$$
l_{\text {eff }}=t_{g} c l / L
$$

with $c$ the speed of light, $l$ and $L$ the lengths of the intracavity absorption cell and of the Ti:Sa laser cavity, respectively. An interesting instrumental challenge in the adaptation of ICLAS to FTS replacing spectrographs as in the usual literature was to synchronize the pulsed character of the technique to the FT sampling procedure. The ULB set-up is described in [58], and applied in [61, 58, 27, 62, 59]. The technique was also developed by other groups (e.g. [63-65]).

\section{Insert figure 12}

As a final picture for the role of anharmonic resonances in the 
bright/dark issue, figure 13 reproduces a DLIF spectrum recorded at MIT under jet-cooled conditions and interpreted at ULB [66]. In the zero order picture, a simple two-line spectrum is expected. The eigenspectrum is, however, composed of numerous peaks. All these additional features happen to originate from an impressive fractionation of the zero order bright transition through many intensity borrowing mechanisms (so-called tier model). The simulation in figure 12 could actually be performed almost independently of the observation, using the so-called cluster picture developed in the next section.

\section{Insert figure 13}

\section{E. Emergence of new constants of the motion}

A key feature emerging from the numerous investigations that were so far highlighted is that the ratios of the vibrational frequencies one to another are close to being integers. These ratios in energy space, mirrored in time space, are obviously isotopologue-dependent. Nevertheless, harmonic frequencies are close enough in all three symmetric isotopologues ${ }^{12} \mathrm{C}_{2} \mathrm{H}_{2},{ }^{13} \mathrm{C}_{2} \mathrm{H}_{2}$ and ${ }^{12} \mathrm{C}^{13} \mathrm{CH}_{2}$, and one can check that, for all three species

$$
1 / 5 \tilde{v}_{1} \approx 1 / 3 \tilde{v}_{2} \approx 1 / 5 \tilde{v}_{3} \approx 1 / 1 \tilde{v}_{4} \approx 1 / 1 \tilde{v}_{5}
$$


Thanks to this relationship, Kelman [67] could introduce an approximate constant of the motion, $N_{r}$ such that

$$
N_{r}=5 v_{1}+3 v_{2}+5 v_{3}+v_{4}+v_{5}
$$

thus directly associated to the ratio of the time periods. All $\left(v_{1}, v_{2}, v_{3}, v_{4}, v_{5}\right)$ states sharing the same $N_{r}$ value, either + or - when $k=0$, either $u$ or $g$ for the symmetric isotopologues, define a vibrational polyad. As pointed out by Zhilinskii [43], one can define a mean vibrational frequency

$$
\tilde{v}_{\text {mean }}=\left(1 / 5 \tilde{v}_{1}+1 / 3 \tilde{v}_{2}+1 / 5 \tilde{v}_{3}+2 / 1 \tilde{v}_{4}+2 / 1 \tilde{v}_{5}\right) / 7
$$

whose value is close to $670 \mathrm{~cm}^{-1}$ in ${ }^{12} \mathrm{C}_{2} \mathrm{H}_{2}$. One can calculate in this isotopologue all $\left(v_{1}, v_{2}, v_{3}, v_{4}, v_{5}\right)$ vibrational state energies within a polyad using the full set of vibrational constants from [24], and reduce them using a weighted procedure accounting for the double degeneracy of all states with $k>0$, to a single average energy, a sort of "centre of gravity" of the polyad $\left(\tilde{v}^{a v}\left(N_{r}\right)\right)$. These reduced energy values obey the simple relation

$$
\tilde{v}^{a v}\left(N_{r}\right)=672.95 N_{r}-0.455 N_{r}^{2}
$$


in which $N_{r}$ thus plays the role of a vibrational quantum number, with a vibrational frequency $\left(672.95 \mathrm{~cm}^{-1}\right)$ quasi-identical to the mean vibrational frequency $\left(670.0 \mathrm{~cm}^{-1}\right)$ defined in (2.18). As also discussed by Kelman [67], $N_{s}$ defines a further invariant of the Hamiltonian, with

$$
N_{s}=v_{1}+v_{2}+v_{3}
$$

As it turns out, the $\left\{N_{s}, N_{r}, k\right\}$ set of numbers, also accounting for +/and $u / g$ symmetries whenever relevant, unequivocally characterizes each of the vibrational polyads. The remarkable feature is that all vibrational states, most likely up to the top of visible energy range if not at even higher energies, fit this model, hence the labelling of superpolyad or cluster assessed at some stage by the MIT and ULB groups, respectively, in the literature. As an example, figure 14 depicts the $\left\{N_{s}, N_{r}, k\right\}=\{2,10,0\}$ cluster in ${ }^{12} \mathrm{C}_{2} \mathrm{H}_{2}$.

\section{Insert figure 14}

This model was used to factorize the acetylene vibrational Hamiltonian, thus into superpolyads or clusters, defining a blockdiagonalised matrix image of the molecule (MIME). The many known vibrational energies were fitted to that MIME, in isotopologue-specific procedures, for ${ }^{12} \mathrm{C}_{2} \mathrm{H}_{2}$ [24], ${ }^{12} \mathrm{C}_{2} \mathrm{D}_{2}[25],{ }^{13} \mathrm{C}_{2} \mathrm{H}_{2}$ [26], ${ }^{12} \mathrm{C}_{2} \mathrm{HD}$ [27], and ${ }^{12} \mathrm{C}^{13} \mathrm{CH}_{2}$ $[39,28]$, each time generating an extended set of vibrational constants. 
These parameters are fully effective. Typically, they allow the observed vibrational energies to be matched within a few $\mathrm{cm}^{-1}$, up to the bottom of the visible range. Figure 15 details the internal structure of the many vibrational energy states in ${ }^{12} \mathrm{C}_{2} \mathrm{H}_{2}$ resulting from this effective but physically well-defined MIME, with respect to $\tilde{v}^{a v}\left(N_{r}\right)$ and the similarly defined $\tilde{v}^{a v}\left(N_{r}, N_{s}\right)$. A remarkable ordering emerges from this picture, quite unexpected if one remembers the large integrated number of vibrational states demonstrated in figure 5 . It must also be emphasized that the width of the various clusters becomes very large with increasing energy, soon much larger than the energy separation between the "centres of gravity" of the clusters.

\section{Insert figure 15}

\section{Global picture.}

Now that a reasonably coherent picture has emerged from the investigation of the vibrational degrees of freedom, one can address the rotational degrees of freedom. In some respects, these were already considered, when including the role of the angular momenta $l_{4}$ and $l_{5}$ in the MIME. The full inclusion of rotation in the MIME defines the so-called global picture. Actually, rotational degrees of freedom were already 
completely and successfully included for various acetylene isotopologues, including several thousands of vibration-rotation lines $[15,22,42,69,70$, 40]. However, only bend states, up to $\sum_{v_{b}} v_{b}=4$, were considered in these procedures. Extension towards high energy stretch-bend states was attempted for ${ }^{12} \mathrm{C}_{2} \mathrm{H}_{2}$ by Perevalov and coworkers, but with limited success [71]. "Real" global procedures, thus over a larger range of vibrational excitations and including rotation, were published only for triatomic species in the literature (see refs in [28]). One should also mention attempts for a five-atom species, methane, ${ }^{12} \mathrm{CH}_{4}$ [72].

The challenge we now face is thus building a global MIME for a molecule larger than triatomic, acetylene, including rotation and valid over a vibrational excitation range as extended as possible. Including rotation obviously means accounting for, and reproducing all existing high resolution data, at their full accuracy.

\section{A. Rotation, notations and conventions}

Several hundreds of publications in the literature since 1936 were devoted to HiReS fingerprints of acetylene in the ground electronic state, as reviewed in $[8,3,9]$. Their results, which formed the basis for the vibrational MIME detailed in the previous section, of course include detailed information on the so far undiscussed rotational structure. This 
structure was already highlighted in previous figures and also in figure 16, this time for ${ }^{12} \mathrm{C}^{13} \mathrm{CH}_{2}$. To illustrate the richness of data such as those presented in figure 16, one should stress that Di Lonardo and coworkers identified more than 70 bands in the full range covered in this figure, thus from 5700 to $10000 \mathrm{~cm}^{-1}[73,74]$, some still to be published [75].

\section{Insert figure 16}

The rotation energies are given by

$$
F(J)=B_{v_{1} v_{2} v_{3} v_{4} v_{5}}\left[J(J+1)-k^{2}\right]-D_{v_{1} v_{2} v_{3} v_{4} v_{5}}\left[J(J+1)-k^{2}\right]^{2}+\ldots
$$

with, for the GS, $\mathrm{B}_{00000}\left(\right.$ or $\mathrm{B}_{0}$ ) the principal rotational constant related to the principal moment of inertia, and $\mathrm{D}_{00000}$ (or $\mathrm{D}_{0}$ ) the lowest order distortion constant. As an example, the vibrational dependence of the principal rotational constants is accounted for in the following way

$$
B_{v}=B_{0}-\sum_{i} \alpha_{i}^{0} v_{i}+\sum_{i \leq j} \gamma_{i j}^{0} v_{i} v_{j}+\sum_{b \leq b^{\prime}} \gamma_{l_{b} b_{b^{\prime}}} l_{b} l_{b^{\prime}}+\sum_{i \leq j \leq m} \varepsilon_{i j m} v_{i} v_{j} v_{m}+\sum_{i, b \leq b^{\prime}} \varepsilon_{i l_{b} b_{b^{\prime}}} v_{i} l_{b} l_{b^{\prime}}
$$

The latest values of $\mathrm{B}_{0}$ are listed for all studied isotopologues in table 3 .

\section{Insert table 3}


It is well known that rotation-vibration wavefunctions can be symmetrized into $e / f$ components, as defined in [80]:

$$
\begin{aligned}
& \left|v_{s}, v_{b}, l_{b}, J, e\right\rangle=\frac{1}{\sqrt{2}}\left[\left|v_{s}, v_{b},\right| l_{b}|, J\rangle+\left|v_{s}, v_{b},-\right| l_{b}|, J\rangle\right] \\
& \left|v_{s}, v_{b}, l_{b}, J, f\right\rangle=\frac{1}{\sqrt{2}}\left[\left|v_{s}, v_{b},\right| l_{b}|, J\rangle-\left|v_{s}, v_{b},-\right| l_{b}|, J\rangle\right]
\end{aligned}
$$

This separation is achieved using the Wang procedure summarized in figure 17, here applied to the (00010) vibrational state selected as an example. The 2 -fold degeneracy of all rotational states within a vibrational state with $k \geq 1$ is lifted when accounting for the so-called rotational $l$-type resonance interaction matrix element

$$
\left\langle l_{b}|\hat{H}| l_{b} \pm 2\right\rangle=1 / 4 q_{b}\left[\left(v_{b} \mp l_{b}\right)\left(v_{b} \pm l_{b}+2\right)\right]^{1 / 2}[J(J+1)-k(k \pm 1)]^{1 / 2}[J(J+1)-(k \pm 1)(k \pm 2)]^{1 / 2}
$$

The expert reader will notice that the convention adopted in Eq. (3.3), that leads to negative $l$-doubling parameters $q_{4}$ and $q_{5}$, is different from most of our own previous literature inputs but the most recent ones. This change is to cope with the vibration-rotation matrix elements used in the computer package building the global model discussed in section $3 \mathrm{C}$.

The resulting rotational structure of selected transition-types is detailed 
in figure 18. It includes the role of nuclear spin statistics adapted to ${ }^{12} \mathrm{C}_{2} \mathrm{H}_{2}$, to be later discussed.

\author{
Insert figures 17 and 18
}

\author{
B. Including rotation in the MIME \\ Rotation can be included in the various vibrational interaction \\ schemes previously discussed, as exemplified in figure 19.

\section{Insert figure 19}

In this figure, the reduced rotational energy is plotted at the right, as a function of $J(J+1)$, as e.g. in $[81,28]$. The value of $J$ rather than $J(J+1)$ is indicated on the graphs for easier reading. This reduced energy is defined as

$$
E(v, J)-\left[B_{\text {sel. }} J(J+1)-D_{0} J^{2}(J+1)^{2}\right]-\tilde{v}
$$

with the value of $\tilde{v}$ selected to perform an adequate translation on the energy axis and avoid dealing with large numbers and the value of $B_{\text {sel. }}$ selected to keep the dependency of the reduced energy of the states of interest as horizontal as possible in the related graphs. If, for instance, the rotational contribution from the GS state is subtracted from the energy, a 
horizontal line is expected for this state while a smooth variation from this linear behaviour is expected to occur for the close vibrational states in these reduced energy graphs, as observed in figure 19.

The energies in figure 19 result from the diagonalization of the adequate MIME, $J$ per $J$-value, built using the related matrix elements, such as appearing in eqs. (2.3), (2.4), (2.5), (3.1), (3.2) and (3.4). The resulting matrix diagonalization leads to the eigenvalues presented in figure 19, and also to the squared coefficients in the eigenvectors associated to each of the zero order, basis states, detailed in figure 20 (see [82] for further details). As illustrated in the latter graphs, a 1:1 mixing occurs in ${ }^{12} \mathrm{C}_{2} \mathrm{H}_{2}$ between these $v_{3}=1$ and $v_{2}=1, v_{4}=1, v_{5}=1, \Sigma^{+}$states. As a first result, the zero order bright $v_{3}$ band intensity is shared with the zero order dark $v_{2}+v_{4}+v_{5}$ transition. As a second consequence, the zero order forbidden $\Delta k=2, \Delta-\Sigma^{+}$transition also emerges, now according to a $J$-dependent mixing controlled by the rotational $l$-type resonance matrix element detailed in Eq. (3.4). The latter effect is specifically highlighted in figure 21 .

\section{Insert figures 20 and 21}

Thus, a partial MIME can be built for this specific interaction scheme, $J$ value by $J$ value, furthermore split into $e$ and $f$ states. Even though the anharmonic resonance coupling mechanisms are $J$ - 
independent (see eq. (2.5)), the rotational constants of the various vibrational states within the same polyad are not identical, thus inducing a variation with $J$ of the energy separation between interacting levels, as highlighted in the example just discussed. As a result, the amplitude of the coupling effectively becomes $J$-dependent. This is a well-known situation which may lead to dramatic effects on the relative band and branch intensities, as e.g. discussed for the pure bends in ${ }^{12} \mathrm{C}_{2} \mathrm{H}_{2}$ in [22]. Another example of coupling is provided in figure 22. It highlights a double avoided crossing affecting the $(00300,00)$ zero order state in ${ }^{12} \mathrm{C}^{13} \mathrm{CH}_{2}$.

\section{Insert figure 22}

\section{Global MIME}

The strategy just highlighted for $v_{3} / v_{2}+v_{4}+v_{5}$ in ${ }^{12} \mathrm{C}_{2} \mathrm{H}_{2}$ could be applied to the full set of data available for this isotopologue. Each extra datum brings another independent piece of relevant information, thus in the end significantly improving the statistical quality of the global fit. On the other hand, rotation-dependent interaction mechanisms show $J$ dependence that will genuinely affect the coupling strength for increasing $J$-values and, again, considering all rotation-vibration data will contribute to better characterize the coupling schemes. Coriolis coupling schemes different from rotational $l$-type resonance were actually identified in a 
number of cases for specific isotopologues, see e.g. [83, 28], though so far not systematically. They are responsible of a limited number of local perturbations and seem not to play a very systematic role in the spectral pattern of acetylene. They are therefore not further addressed in this review.

A global MIME exploiting the vibrational cluster structure and accounting for the $l$-dependent coupling terms was built to deal not only with pure bending states in various isotopologues, as previously mentioned. It was extended to the full set of available rotation-vibration data in ${ }^{12} \mathrm{C}^{13} \mathrm{CH}_{2}$. The reason for selecting this specific isotopologue mainly arises from the extensive set of data available. Since there is no restrictive $u / g$ selection rule, indeed, many more transitions are allowed, compared to the symmetric ${ }^{12} \mathrm{C}_{2} \mathrm{H}_{2}$ species. Another consequence of this absence of symmetry is that the interaction scheme is also more complete, as illustrated in figure 23. These specific features, compared to ${ }^{12} \mathrm{C}_{2} \mathrm{H}_{2}$, define additional constraints helping the fitting procedure to converge to a single set of parameters, only.

\section{Insert figure 23}

The systematic investigation of ${ }^{12} \mathrm{C}^{13} \mathrm{CH}_{2}$ using FT and FT-ICLAS spectroscopy, in particular, allowed several thousands of rotationvibration lines to be assigned, up to the near infrared region (e.g. [70, 84, 
$62,73,74]$. Thus $\left\{N_{s}, N_{r}, J, e / f\right\}$ clusters were built for each $J$-value, with the pseudo quantum numbers $N_{s}$ and $N_{r}$ defined as in (2.20) and (2.17), respectively. The $k$-off diagonal matrix elements only couple states differing by 2 in $k$ and thus only even or odd $k$-states are present within a single global cluster. A typical cluster matrix, with $\left\{N_{s}, N_{r}\right\}=\{2,10\}$, is illustrated in figure 24, only reproducing part of the full Hamiltonian. Higher order terms defined in [85, 28] are included in the off-diagonal coupling elements.

\section{Insert figure 24}

A fit was performed in [28] considering all 12703 vibrationrotation lines assigned in ${ }^{12} \mathrm{C}^{13} \mathrm{CH}_{2}$ up to $6750 \mathrm{~cm}^{-1}$, weighted according to the stated accuracy of the line positions (typically between $10^{-2}$ and $10^{-4}$ $\mathrm{cm}^{-1}$ ). Actually the limit was even systematically slightly decreased to improve the quality of the fit. These lines reach about 4350 rotation states from 158 different $v / k$ vibrational states. The fit of 11738 of these lines was successfully achieved with a dimensionless standard deviation of 0.99 using 216 parameters. The set of parameters include $K_{11 / 33}, K_{1 / 244}$, $K_{1 / 245}, K_{1 / 255}, K_{3 / 244}, K_{3 / 245}, K_{3 / 255}, K_{14 / 35}, K_{15 / 34}$ and $K_{44 / 55}$ for the anharmonic resonances. $\underline{\text { All }} 965$ remaining lines in the ${ }^{12} \mathrm{C}^{13} \mathrm{CH}_{2}$ fit being discussed, thus those left outside from the procedure, are recalculated within the initially stated wavenumber position accuracy, before reducing it to improve the quality of the fit. It thus turns out that 
the cluster model, extended to include rotation, allows all assigned lines up to $6750 \mathrm{~cm}^{-1}$ in ${ }^{12} \mathrm{C}^{13} \mathrm{CH}_{2}$ to be satisfactorily reproduced, within or better than the stated typical HiReS position accuracy. It is indeed, so far, a successful story.

A key issue for the success of the global analysis, besides the spectral analysis procedure already mentioned in the text, was the package of highly efficient computer programs with very effective graphical output achieved by Fayt and co-workers in Louvain (see e.g. [86, 81]).

The predictive power of the global model just discussed for ${ }^{12} \mathrm{C}^{13} \mathrm{CH}_{2}$ proved critical in assigning some of the bands in the range considered in the work just referred to. Actually 17 bands previously unspotted because of their low intensity or not analysed because of their highly perturbed rotational structure, could be identified and assigned thanks to the global model, as discussed in [28]. This global procedure is now being extended for ${ }^{12} \mathrm{C}^{13} \mathrm{CH}_{2}$ towards higher excited regions, so far with success [75]. It is also being applied, again so far successfully, to ${ }^{12} \mathrm{C}_{2} \mathrm{H}_{2}$ [87]. Meanwhile, it can be used to unravel various features in the observed spectra of both isotopologues. As an example, figure 25 details the predicted set of vibration-rotation states in a selected range in ${ }^{12} \mathrm{C}^{13} \mathrm{CH}_{2}$, at high vibrational excitation. Several avoided crossings can be spotted and assigned from such graphs, as a possible help towards the detailed spectral analysis. 
This complex behaviour also illustrates the need for a more adequate basis to deal with overtone states.

\section{Insert figure 25}

The next step in this global process is to reliably reproduce the spectrum including relative and hopefully absolute intensity features. Several investigations were carried on in the literature concerning intensity simulations, however limited to sharply defined selected spectral ranges (see e.g. [71]). We have also considered particular problems, such as the determination of the transition dipole moment of $v_{5}-v_{4}$ in ${ }^{12} \mathrm{C}_{2} \mathrm{H}_{2}$, of relevance to space missions [40]. We are now attempting to simulate a range as extended as possible, using the results of the global MIME to account for all relevant couplings and produce reliable intensity predictions. This step involves various model refinements, considering the sign of the resonance parameters, in particular.

Another application of the global model is the spectral simulation of absorption data at very high temperatures for astrophysical purposes, thus back to the fingerprint issue of HiReS. Such a simulation requires the a priori global knowledge of all state energies and coupling schemes, while the zero order transition moments and their relative signs need to be carefully estimated to reliably reproduce the intensity features. As a flavour to these forthcoming developments, figure 26 shows a simulated 
absorption spectrum in the range of $v_{5}$ in ${ }^{12} \mathrm{C}_{2} \mathrm{H}_{2}$, under $500 \mathrm{~K}$ temperature conditions, thus accounting for all relevant hot bands and related upper state coupling mechanisms.

\section{Insert figure 26}

Dreaming that such a spectral simulation can be systematically achieved, say on a range as extended as from 0 to $15000 \mathrm{~cm}^{-1}$, it would cover the equivalent of a pulsed excitation of about $1 \mathrm{fs}$ on the time scale. This is another quite peculiar perspective stimulating the present purely spectroscopic strategy of research. Altogether, the need for an appropriate description of the highly excited vibration-rotation states, merging quantum, dynamical and statistical issues, is still to be imagined.

\section{Ultimate MIME}

As a last input in this review, one should remember that, thanks to the Pauli principle, nuclear spin isomers need be defined for the symmetric acetylene isotopologues. Figure 27 summarizes the situation, with the states with highest and lowest nuclear spin statistics labelled ortho and para, respectively. The well known spectral intensity alternation with $J$, illustrated in figure 28 is one of the striking consequences of the existence of nuclear spin isomers in acetylene. It 
applies to all transitions in the symmetric isotopologues, with ortho to para state transitions forbidden, as already illustrated in figure 18 . The global MIME ought obviously to include the role of nuclear spins, hence the title "ultimate MIME" to the present subsection.

\section{Insert figures 27 and 28}

Literature on the role of spin-rotation interactions in acetylene, that would be most relevant in this context, is lacking. We shall therefore here only highlight some thoughts on related issues considering $(i)$ nuclear spin conversion, (ii) partition functions, (iii) collision processes and, finally (iv) formation of molecular complexes.

(i) Nuclear spin conversion:

Despite several conversion attempts between ortho and para species (e.g. $[89-92,9])$, it seems that such a nuclear spin isomerization process was not observed in acetylene, so far. As a matter of fact, although it has been investigated in some species, with interesting applications (e.g. [93, 94]), this kind of conversion is known to be usually negligible, given the smallness of the nuclear spin interaction terms that mix wavefunctions from different nuclear spin isomers. The strategy to investigate nuclear spin conversion first relies on the capacity to enrich a sample in one of the isomers, before studying the return to statistical equilibrium. We recently 
reported [95] on a possible enrichment procedure, though accidental and most indirect. We indeed noticed, as reported in figure 29 , that the absorption line profile of the first $R(J)$ transitions in the $v_{1}+v_{3}$ band of ${ }^{12} \mathrm{C}_{2} \mathrm{H}_{2}$ recorded with a tunable diode laser in an axisymmetric jet presented a central dip. It could possibly partly due to the formation of van der Waals species that is likely to be more favourable in the colder, central part of the jet beam thus removing monomer absorption. The disappearance of this dip with increasing rotation, still to be confirmed could possibly indicate that rotation hinders the formation of these aggregates thus leading to recover the full amount of monomers. As a result, the beam would then be specifically depleted in species with $J=0$, i.e. in para species. Thus very indirect enrichment in ortho species might possibly occur in jet-cooled acetylene, under conditions used in the experiments! As pointed out this interpretation is still most tentative and calls for strict experimental evidence.

\section{Insert figure 29}

(ii) Partition functions

The calculation of absolute line parameters and various other properties rely on partition functions $(Q)$. Since ortho and para species do not easily convert into each other, they ought to be considered as different species in a number of processes and issues, including partition functions, 
as already stated e.g. by Dennison in 1927 \{Dennison, 1974 \#4631\}. The expressions for the two forms are presented in Eq. (3.6).

$$
\begin{aligned}
& Q_{\text {ortho }}=3 \sum_{J_{\text {ortho }}} g_{J_{\text {ortho }}} \exp \left[-\left(E_{v_{\text {ortho }}}-E_{G S, J=1}\right) / k T\right] \\
& Q_{\text {para }}=1 \sum_{J_{\text {para }}} g_{J_{\text {para }}} \exp \left[-\left(E_{v_{\text {p }} \text { para }}-E_{G S, J=0}\right) / k T\right]
\end{aligned}
$$

In these expressions we accounted for different lowest energy states for the ortho and para nuclear isomers, respectively $J=1$ and $J=0$, to stress the need for considering these two species separately. Obviously, the proper calculation of the thermal population of each of the rotational states, as performed in figure 26, requires the same selection for the reference energies. The usual factor $g_{J}(=2 J+1)$ in these expressions correspond to the spatial degeneracy.

The connection towards the usual partition function, as defined for a single acetylene species ${ }^{12} \mathrm{C}_{2} \mathrm{H}_{2}$ in most books, is achieved in Eqs. (3.7) and (3.8). The distinction between different initial rotational states for the two isomers is not made in these equations. First, one needs to merge the contribution of both isomers, accounting for their respective abundance under equilibrium conditions: 


$$
\begin{aligned}
Q_{\text {Total }}= & 3 / 4 \sum_{J_{\text {ortho }}} g_{J_{\text {ortho }}} \exp \left[-\left(E_{v, J_{\text {ortho }}}-E_{G S}\right) / k T\right] \\
& +1 / 4 \sum_{J_{\text {para }}} g_{J_{\text {para }}} \exp \left[-\left(E_{v, J_{\text {para }}}-E_{G S}\right) / k T\right]
\end{aligned}
$$

Next, assuming high temperature conditions, the sums on ortho and para states are each about identical, therefore leading to the final expression, with the factor $1 / 2$ presented as the symmetry factor in the usual pedagogical literature.

$$
Q_{\text {Total }}^{T \square} \approx 1 / 2 \sum_{J} g_{J} \exp \left[-\left(E_{v, J}-E_{G S}\right) / k T\right]
$$

Obviously, the proper calculation of the thermal population of each of the rotational states, as performed in figure 26 , requires the same reference energies, different for ortho and para specie.

(iii) Collision processes

We have only dealt with intramolecular features, so far. One can, however, expect intermolecular issues to be affected by intramolecular coupling schemes. Acetylene probably offers unique opportunities to investigate how several of the features highlighted in this review may influence or transfer into collision processes and aggregation mechanisms, including nucleation. 
Concerning collisions, several line profile investigations were reported in the literature in various groups (see review in [97]). We shall only report here, as an illustration, on the abnormal self-pressure shift effects affecting $J=18$ in $v_{1}+3 v_{3},{ }^{12} \mathrm{C}_{2} \mathrm{H}_{2}$. This specific vibration-rotation state exhibits intramolecular perturbation and, as a result the $R(17)$ and $P(19)$ lines accessing that state are doubled as reported in several papers including [98, 52, 99-101, 61]. The situation is schematized in figure 30 . At the time [61], the available global constants allowed first to unravel the zero order composition of the $v_{1}+3 v_{3}$ vibrational state, as indicated in figure 30. Next, they suggested that the local perturbation affecting $J=18$ could be assigned to a Coriolis-type, thus $J$-dependent interaction with a state of $\Pi$ symmetry involving significant bend excitation, also identified, tentatively, on figure 30 . As a result of the latter mixing, this vibrationrotation state from a zero order pure stretch state carries bending character. Since, in the overtone range, vibrationally-induced changes of the mean geometrical structure become more significant, it is reasonable to suggest that the collision mechanisms are significantly affected by introducing this bend character, hence the different shift coefficients measured for the lines reaching this $J=18$ state in the $v_{1}+3 v_{3}$ band in ${ }^{12} \mathrm{C}_{2} \mathrm{H}_{2}$. Once obtained, the new global constant in this isotopologue, this time including rotational degrees of freedom will be used to check the nature of this specific wavefunction mixing. Actually, this statement about geometrical structure changes in the overtone region supported unfortunately unsuccessful attempts to perform laser drift experiments in 
acetylene in connection with nuclear spin conversion investigation [89].

\section{Insert figure 30}

(iv) Molecular complexes

Concerning formation of complexes, we shall not review here the literature around acetylene-containing van der Waals species, dimers and multimers (see e.g. [9]). We shall only present two very recent results obtained at ULB, illustrated in figures 31 and 32 . The first is the observation of the ${ }^{12} \mathrm{C}_{2} \mathrm{H}_{2}-\mathrm{Ar}$ complex at high resolution around $6500 \mathrm{~cm}^{-1}$ using cavity ring down spectroscopy [102], and the second is the probable observation of multimers of ${ }^{12} \mathrm{C}_{2} \mathrm{H}_{2}$, possibly containing Ar, around 3300 $\mathrm{cm}^{-1}$ using Fourier transform spectroscopy ([103]).

Insert figures 31 and 32

We take this opportunity to highlight, following the FT recording of coloured vibrations and the 2T-FT-ICLAS achievements previously illustrated, another instrumental development stimulated at ULB by the acetylene saga. The set-up used to record the spectra presented in figures 29, 31 and 32 is the next generation instrument to produce and interrogate supersonic expansions. Our previous set-up combining a slit jet and FTS proved to be very performing thanks to the use of a $16 \mathrm{~cm}$ long 
slit or multi nozzle (see [104] for a literature review on FT-jet spectroscopy). A major drawback was the amount of gas injected, which prevented us from using acetylene given its hazardous character. We have very recently built a new set-up, named FANTASIO for "Fourier trANsform, Tunable diode and quadrupole mAss spectrometers interfaced to a Supersonic expansIOn”. The injection, through an axisymmetic or slit nozzle, is now compatible with the use of acetylene, while a large turbomolecular pumping group allows improved stagnation to residual pressure ratios to be achieved. The sensitivity is boosted thanks to a multipass sytem around the jet for FTS recording over very broad spectral ranges and to thanks a cavity ring down system for tunable diode laser investigations in the $1.5 \mu \mathrm{m}$ spectral region. Besides the recent detection of complexes, as illustrated in figures 31 and 32 , we are on our way measuring absolute line intensities under jet cooled conditions [105]. We also plan to inject a pump laser at the nozzle exit to perform double resonance spectroscopy, hopefully also investigating the detailed mechanism of acetylene nucleation.

\section{Final words}

The present paper was intended to respond to the invitation to present the ULB contribution to the acetylene ground state investigation, 
or so-called acetylene saga. One should obviously grant the contribution of many other research groups to this saga, several of them identified in the introduction, whose contribution was accounted for in a previous review [8]. The central issue in the acetylene saga as defined in the present paper is the understanding, at the HiReS level of accuracy, of the molecular behaviour in regions of high vibrational excitation. Many basic questions remain, including an appropriate zero order description involving interplay between quantum, classical and dynamical pictures. The probably complex response still to come is a key issue to unravel and to master a number of important chemical mechanisms at the microscopic level of investigation.

The research strategy along the acetylene saga fully relies on HiReS, stimulating instrumental developments and, altogether generating an impressive amount of first quality data also feeding more traditional fingerprint type applications of HiReS. It is indeed interesting to point out that all spectral data in the overtone ranges, initially generated to help very fundamental research projects, today also support applied issues such as trace detection relying on near infrared laser developments. The acetylene saga thus contributed promoting HiReS as a major input to modern and hot topics. This is a quite rewarding status in this period of Science favouring interdisciplinary research....and often dismissing HiReS.

The acetylene saga is far from ended, and the so-called ultimate 
MIME in section 3D is, for sure, far from ultimate. The various research tracks and questions that arose in the text and many others to come will keep stimulating the activity of many research groups, including ours. 


\section{Acknowledgments}

I wish to thank Prof. F. Merkt (ETH-Zurich), editor of Molecular Physics, for inviting me to submit this review. It would simply not have existed without the input of several collaborators and friends all over the world. I wish to highlight the acetylene co-authors active in Brussels during this saga, namely Profs M. Godefroid, J. Liévin and Dr. J. Vander Auwera, as well as Prof. P. Gaspard; also, as PhD students either at the time or still now, M. Abbouti Temsamani, C. Depiesse, K. Didriche, I. El Idrissi, F. Herreggodts, T. Huet, D. Hurtmans, C. Lauzin, S. Robert and, as postdoc researchers, M. Hepp, Y. Kabbadj, S. Kassi and P. Macko. It is also my pleasure to specifically thank Dr A. Campargue, from Grenoble, Profs G. Di Lonardo and L. Fusina, from Bologna, Prof. A. Fayt from Louvain, Prof. B.J. Orr, from Sydney, and Prof. B. Zhilinskii from Dunkerque, for a very friendly collaboration about acetylene. I also thank Prof. Di Lonardo (Bologna) for providing unpublished results listed in the present paper, Drs Coheur and Herbin (ULB) for the figure on atmospheric acetylene, Dr Demaison (Lille) for pointing out reference [96], Prof. Gaspard (ULB) for useful comments on partition functions, Prof. Fayt (Louvain) and Séverine Robert (Brussels), in particular, not forgetting Keevin Didriche and Badr Amyay (Brussels) for various figures. Séverine Robert and Dr Vander Auwera (ULB) also kindly commented on an earlier version of the manuscript, while Profs Di Lonardo (Bologna), Fayt (Louvain), Sutcliffe (Brussels) and Zhilinskii (Dunkerque) all reviewed the final version. 
Eventually, I wish to acknowledge the constant financial support of the Belgian FRS-FNRS along these acetylene years as well as, more specific to the present time period, support from the "Actions de recherches Concertées de la Communauté française de Belgique", the "Laboratoire Européen Associé HiReS", and the "European Union" (Quantitative Spectroscopy for Atmospheric and Astrophysical Research, QUASAAR, contract MRTN-CT-2004-512202). 


\section{References}

1. S. A. Miller, "Acetylene; Its properties, manufacture and uses, vol. I" Ernest Benn Limited, London, (1965).

2. E. Davy, British. Ass. Reports, 62, (1836).

3. M. Herman, A. Campargue, M. I. El Idrissi, and J. Vander Auwera, J. Phys. Chem. Ref. Data, 32, 921 (2003).

4. J. Demaison, private communication.

5. P. F. Coheur, B. Barret, S. Turquety, D. Hurtmans, J. Hadji-Lazaro, and C. Clerbaux, J. Geophys. Res., 110, D24303 (2005).

6. P. F. Coheur, H. Herbin, D. Hurtmans, C. Wespes, C. Clerbaux, C. D. Boone, P. F. Bernath, C. P. Rinsland, and J. Remedios, Geophys. Res. Letters, submitted (2007).

7. M. Herman, D. Hurtmans, J. Vander Auwera, and M. Vervloet, J. Mol. Spectrosc., 150, 293 (1991).

8. M. Herman, J. Liévin, J. Vander Auwera, and A. Campargue, Adv. Chem. Phys., 108, 1 (1999).

9. B. J. Orr, Int. Rev. Phys. Chem., 25, 655 (2006).

10. R. Colin, M. Herman, and I. Kopp, Mol. Phys., 37, 1397 (1979).

11. M. Herman and R. Colin, Phys. Scr., 25, 275 (1982).

12. J. K. G. Watson, M. Herman, J. C. Van Craen, and R. Colin, J. Mol. Spectrosc., 95, 101 (1982).

13. J. C. Van Craen, M. Herman, and R. Colin, J. Mol. Spectrosc., 111, 185 (1985).

14. J. C. Van Craen, M. Herman, R. Colin, and J. K. G. Watson, J. Mol. 
Spectrosc., 119, 137 (1986).

15. T. R. Huet and M. Herman, J. Mol. Spectrosc., 132, 361 (1988).

16. T. R. Huet and M. Herman, J. Mol. Spectrosc., 137, 396 (1989).

17. J. Vander Auwera, T. R. Huet, M. Herman, C. D. Hamilton, and R. W. Field, J. Mol. Spectrosc., 137, 381 (1989).

18. T. R. Huet, M. Godefroid, and M. Herman, J. Mol. Spectrosc., 144, 32 (1990).

19. J. Vander Auwera, T. R. Huet, M. Abbouti Temsamani, and M. Herman, J. Mol. Spectrosc., 148, 93 (1991).

20. M. Herman, T. R. Huet, and M. Vervloet, Mol. Phys., 66, 333 (1989).

21. M. Herman and T. R. Huet, Mol. Phys., 70, 545 (1990).

22. M. Herman, T. R. Huet, Y. Kabbadj, and J. Vander Auwera, Mol. Phys., 72, 75 (1991).

23. T. R. Huet, M. Herman, and J. W. C. Johns, J. Chem. Phys., 94, 3407 (1991).

24. M. I. El Idrissi, J. Liévin, A. Campargue, and M. Herman, J. Chem. Phys., 110, 2074 (1999).

25. M. Herman, M. I. El Idrissi, A. Pisarchik, A. Campargue, A.-C. Gaillot, L. Biennier, G. Di Lonardo, and L. Fusina, J. Chem. Phys., 108, 1377 (1998).

26. G. Di Lonardo, L. Fusina, E. Venuti, J. W. C. Johns, M. I. El Idrissi, J. Liévin, and M. Herman, J. Chem. Phys., 111, 1008 (1999).

27. M. Herman, C. Depiesse, G. Di Lonardo, A. Fayt, L. Fusina, D. Hurtmans, S. Kassi, M. Mollabashi, and J. Vander Auwera, J. Mol. Spectrosc., 228, 499 (2004).

28. A. Fayt, S. Robert, C. Di Lonardo, L. Fusina, F. Tamassia, and M. Herman, J. Chem. Phys., 126, 114303 (2007).

29. E. Venuti, G. Di Lonardo, P. Ferracuti, L. Fusina, and I. M. Mills, Chem. 
Phys., 190, 279 (1995).

30. B. I. Zhilinskii, M. I. El Idrissi, and M. Herman, J. Chem. Phys., 113, 7885 (2000).

31. P. Jensen, Mol. Phys., 98, 1253 (2000).

32. T. Arusi-Parpar, R. P. Schmid, R. J. Li, I. Bar, and S. Rosenwaks, Chem. Phys. Lett., 268, 163 (1997).

33. M. Abbouti Temsamani and M. Herman, Chem. Phys. Lett., 260, 253 (1996).

34. J. Liévin, M. Abbouti Temsamani, P. Gaspard, and M. Herman, Chem. Phys., 190, 419 (1995).

35. M. Herman, M. Abbouti Temsamani, D. Lemaitre, and J. Vander Auwera, Chem. Phys. Lett., 185, 220 (1991).

36. R. R. Hall, Laser Photoacoustic Spectroscopy of forbidden transitions: Acetylene and alkyne high energy vibrational states and their interactions. 1984, PhD thesis, Rice University, Houston-TX, USA.

37. T. Gherman, S. Kassi, A. Campargue, and D. Romanini, Chem. Phys. Lett., 383, 353 (2004).

38. K. K. Lehmann, J. Chem. Phys., 79, 1098 (1983).

39. S. Robert, A. Fayt, G. Di Lonardo, L. Fusina, F. Tamassia, and M. Herman, J. Chem. Phys., 123, 174302/1 (2005).

40. S. Robert, M. Herman, J. Vander Auwera, G. Di Lonardo, L. Fusina, G. Blanquet, M. Lepère, and A. Fayt, Mol. Phys., 105, 587 (2007); erratum (submitted).

41. S. A. B. Solina, J. P. O'Brien, R. W. Field, and W. F. Polik, J. Phys. Chem., 100, 7797 (1996).

42. Y. Kabbadj, M. Herman, G. Di Lonardo, L. Fusina, and J. W. C. Johns, J. 
Mol. Spectrosc., 150, 535 (1991).

43. M. I. El Idrissi, B. Zhilinskii, P. P. Gaspard, and M. Herman, Mol. Phys., 101, 595 (2003).

44. A. Campargue, M. Abbouti Temsamani, and M. Herman, Mol. Phys., 90, 793 (1997).

45. S. F. Yang, L. Biennier, A. Campargue, M. Abbouti Temsamani, and M. Herman, Mol. Phys., 90, 807 (1997).

46. G. Weirauch, A. Campargue, M. I. El Idrissi, and M. Herman, Mol. Phys., 99, $143(2001)$.

47. G. Weirauch, M. I. El Idrissi, J. Vander Auwera, M. Herman, and A. Campargue, Mol. Phys., 99, 969 (2001).

48. Y. Ding, M. Herman, and A. Campargue, J. Mol. Spectrosc., 212, 125 (2002).

49. A. Campargue, E. Bertseva, and Y. Ding, J. Mol. Spectrosc., 220, 13 (2003).

50. A. Campargue, L. Wang, P. Cermak, and S.-M. Hu, Chem. Phys. Lett., 403, 287 (2005).

51. X. Zhan and L. Halonen, J. Mol. Spectrosc., 160, 464 (1993).

52. X. Zhan, O. Vaittinen, and L. Halonen, J. Mol. Spectrosc., 160, 172 (1993).

53. J. Sakai, H. Segawa, and M. Katayama, J. Mol. Spectrosc., 164, 580 (1994).

54. M. Becucci, E. Castellucci, L. Fusina, G. Di Lonardo, and H. W. Schrötter, J. Raman Spectrosc., 29, 237 (1998).

55. D. Bermejo, G. Di Lonardo, J. L. Domenech, and L. Fusina, J. Mol. Spectrosc., 219, 290 (2003).

56. J. Vander Auwera, J. Mol. Spectrosc., 201, 143 (2000).

57. F. Herregodts, E. Kerrinckx, T. R. Huet, and J. Vander Auwera, Mol. Phys., 101, 3427 (2003). 
58. S. Kassi, C. Depiesse, M. Herman, and D. Hurtmans, Mol. Phys., 101, 1155 (2003).

59. P. Macko and M. Herman, Chem. Phys. Lett., 417, 471 (2006).

60. C. Domingo, A. Del Olmo, R. Escribano, D. Bermejo, and J. M. Orza, J. Chem. Phys., 96, 972 (1992).

61. D. Hurtmans, S. Kassi, C. Depiesse, and M. Herman, Mol. Phys., 100, 3507 (2002).

62. C. Depiesse, G. D. Lonardo, A. Fayt, L. Fusina, D. Hurtmans, S. Robert, F. Tamassia, J. Vander Auwera, A. Baldan, and M. Herman, J. Mol. Spectrosc., 229, 137 (2005).

63. S. Hu, H. Lin, S. He, J. Cheng, and Q. Zhu, Phys. Chem. Chem. Phys., 1, 3727 (1999).

64. N. Picque, Vib. Spectrosc., 873, 1 (2002).

65. S. Yang, M. Metsala, T. Lantta, P. Suero, R. Martinez, O. Vaittinen, and L. Halonen, Chem. Phys. Lett., 396, 213 (2004).

66. M. Abbouti Temsamani, M. Herman, S. A. B. Solina, J. P. O'Brien, and R. W. Field, J. Chem. Phys., 105, 11357 (1996).

67. M. Kelman, J. Chem. Phys., 93, 6630 (1990).

68. M. Abbouti Temsamani, Acetylene, $\tilde{X}^{1} \Sigma_{g}^{+}$: from high resolution spectroscopy to intramolecular dynamics, 1996, PhD thesis, ULB, Belgium.

69. E. Canè, G. Cazzoli, G. Di Lonardo, L. Dore, R. Escribano, and L. Fusina, J. Mol. Spectrosc., 216, 447 (2002).

70. G. Di Lonardo, A. Baldan, G. Bramati, and L. Fusina, J. Mol. Spectrosc., 213, 57 (2002).

71. V. I. Perevalov, S. A. Tashkun, O. M. Lyulin, and J.-L. Teffo, NATO Security 
through Science Series C: Environmental Security "Remote Sensing of the Atmosphere for Environmental Security", Springer 139 (2006).

72. V. Boudon, M. Rey, and M. Loëte, JQSRT, 98, 394 (2006).

73. E. Cané, L. Fusina, F. Tamassia, A. Fayt, M. Herman, S. Robert, and J. Vander Auwera, Mol. Phys., 104, 515 (2006).

74. G. Di Lonardo, L. Fusina, F. Tamassia, A. Fayt, S. Robert, J. Vander Auwera, and M. Herman, Mol. Phys., 104, 2617 (2006).

75. G. Di Lonardo, L. Fusina, F. Tamassia, A. Fayt, D. Robert, J. Vander Auwera, and M. Herman, unpublished results, (2007).

76. G. Di Lonardo and L. Fusina, (unpublished results).

77. G. Di Lonardo, P. Ferracuti, L. Fusina, E. Venuti, and J. W. C. Johns, J. Mol. Spectrosc., 161, 466 (1993).

78. G. Wlodarczak, J. Demaison, J. Burie, and M. C. Lasne, Mol. Phys., 66, 669 (1989).

79. M. Huhanantti, J. Hietanen, R. Anttila, and J. Kauppinen, Mol. Phys., 37, 905 (1979).

80. J. M. Brown, J. T. Hougen, K. P. Huber, J. W. C. Johns, I. Kopp, H. LefebvreBrion, A. J. Merer, D. A. Ramsay, J. Rostas, and R. N. Zare, J. Mol. Spectrosc., 55, 500 (1975).

81. A. Fayt, C. Vigouroux, F. Willaert, L. Margules, L. F. Constantin, J. Demaison, G. Pawelke, El Bachir Mkadmi, and H. Buerger, J. Mol. Struct., 695-696, 295 (2004).

82. J. Vander Auwera, D. Hurtmans, M. Carleer, and M. Herman, J. Mol. Spectrosc., 157, 337 (1993).

83. L. Fusina, F. Tamassia, and G. Di Lonardo, Mol. Phys., 103, 2613 (2005). 
84. L. Fusina, G. Bramati, A. Mazzavillani, and G. Di Lonardo, Mol. Phys., 101, $513(2002)$.

85. G. Di Lonardo and L. Fusina, private communication, (2007).

86. C. Vigouroux, A. Fayt, A. Guarnieri, A. Huckauf, H. Burger, D. Lentz, and D. Preugschat, J. Mol. Spectrosc., 202, 1 (2000).

87. S. Robert, B. Amyay, M. Herman, and A. Fayt, unpublished results, (2007).

88. M. Herman and J. Lievin, J. Chem. Edu., 59, 17 (1982).

89. P. Macko and M. Herman, Unreported experiments.

90. A. P. Milce and B. J. Orr, J. Chem. Phys., 112, 9319 (2000).

91. M. Metsälä, M. Nela, S. Yang, O. Vaittinen, and L. Halonen, Vib. Spectrosc., 29, 155 (2002).

92. M. Metsälä, S. Yang, O. Vaittinen, and L. Halonen, J. Chem. Phys., 117, 8686 (2002).

93. P. Cacciani, J. Cosléou, F. Herlemont, M. Khelkhal, and J. Lecointre, Phys. Rev. A, 69, 032704 (2004).

94. M. Tudorie, P. Cacciani, J. Cosléou, F. Herlement, M. Khelkhal, C. Puzzarini, S. Maret, and C. Kahane, A\&A, 453, 755759 (2006).

95. M. Herman, K. Didriche, D. Hurtmans, B. Kizil, P. Macko, A. Rizopoulos, and P. Van Poucke, Mol. Phys., 105, 843 (2007).

96. D. M. Dennison, American Journal of Physics, 42, 1051 (1974).

97. M. Lepère, G. Blanquet, J. Walrand, J. P. Bouanich, M. Herman, and J. Vander Auwera, J. Mol. Spectrosc., 242, 25 (2007)).

98. B. C. Smith and J. S. Winn, J. Chem. Phys., 94, 4120 (1991).

99. F. Herregodts, M. Hepp, D. Hurtmans, J. Vander Auwera, and M. Herman, J. Chem. Phys., 111, 7961 (1999). 
100. F. Herregodts, D. Hurtmans, J. Vander Auwera, and M. Herman, J. Chem. Phys., 111, 7954 (1999).

101. F. Herregodts, D. Hurtmans, J. Vander Auwera, and M. Herman, Chem. Phys. Lett., 316, 460 (2000).

102. P. Macko, C. Lauzin, and M. Herman, unpublished results (2007).

103. Y.-C. Lee, V. Venkatesan, Y.-P. Lee, P. Macko, K. Didriche, and M. Herman, Chem. Phys. Lett., 435, 247 (2007).

104. M. Herman, R. Georges, M. Hepp, and D. Hurtmans, Int. Rev. Phys. Chem., 19, 277 (2000).

105. K. Didriche, P. Macko, M. Herman, J. Thiévin, A. Benidar, and R. Georges, JQSRT, 105, 128 (2007).

106. S. Robert, $\mathrm{PhD}$ Thesis, ULB, in progress. 


\section{Figures}

Figure 1: Portion of the transmittance FT spectrum of the acetylene recorded from space on October, 82005 above Mozambique (-6.85 ${ }^{\circ}$ lat; $39.42^{\circ}$ long.) during the ACE mission (from $[5,6]$ ). The upper spectrum shows the simulated spectrum, the middle one presents the data as recorded from space and the bottom spectrum shows the difference with the spectrum calculated under observation conditions, the latter however not including the acetylene contribution. This contribution is thus highlighted in the bottom spectrum. Acetylene lines that seem to be missing are actually buried into fully saturated regions.

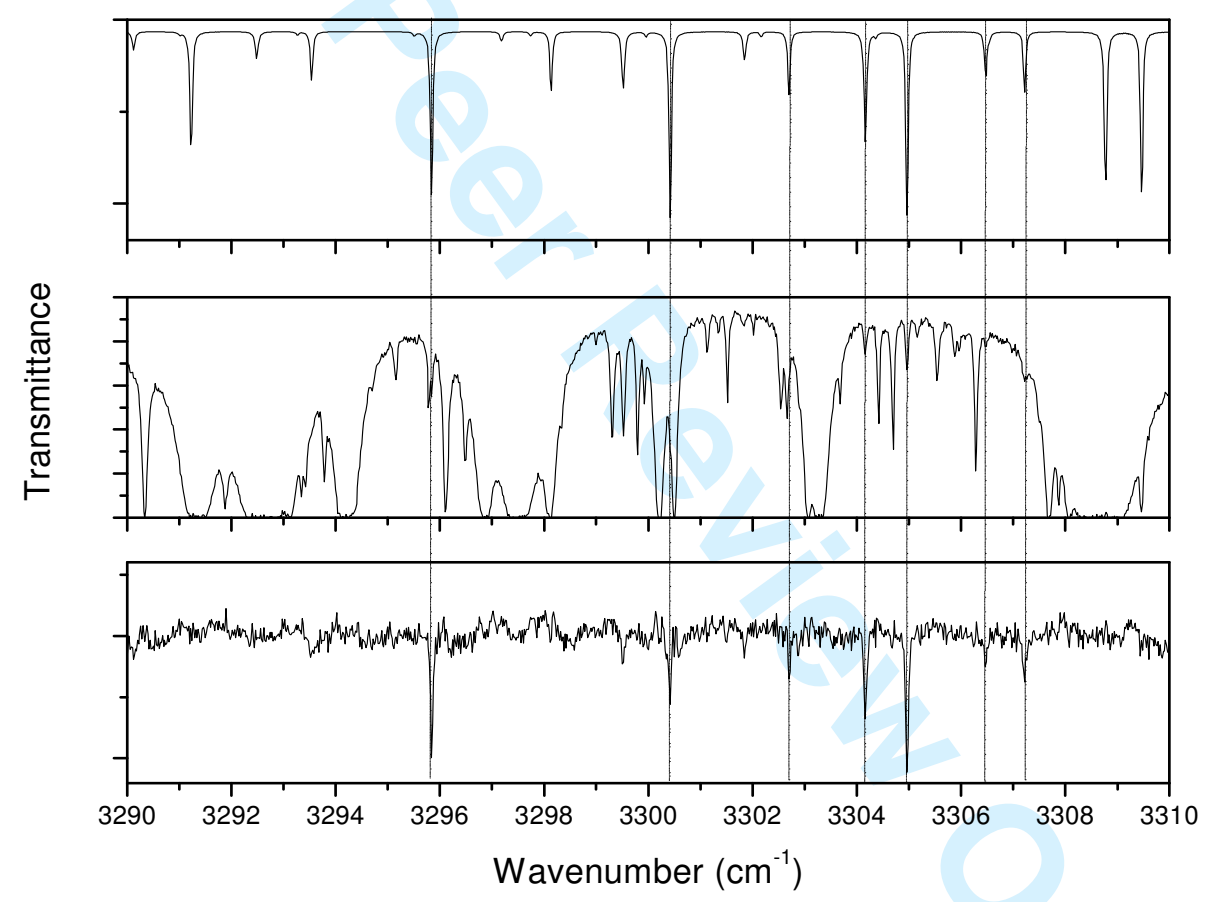


Figure 2: Portion of the emission spectrum of ${ }^{12} \mathrm{C}_{2} \mathrm{H}_{2}$ showing part of the $R$ branches of the $v_{3}$ and $v_{2}+v_{4}+v_{5}$ bands, recorded in emission from a discharge (a). Intensity alternation is absent for those lines pointed out by arrows, as highlighted from the comparison with an absorption spectrum (b) (see dots). The figure is adapted from [7]. It is shown in this reference that this phenomenon can be fully explained by a succession of emission/absorption processes, allowing the emission temperature to be inferred from the observations.
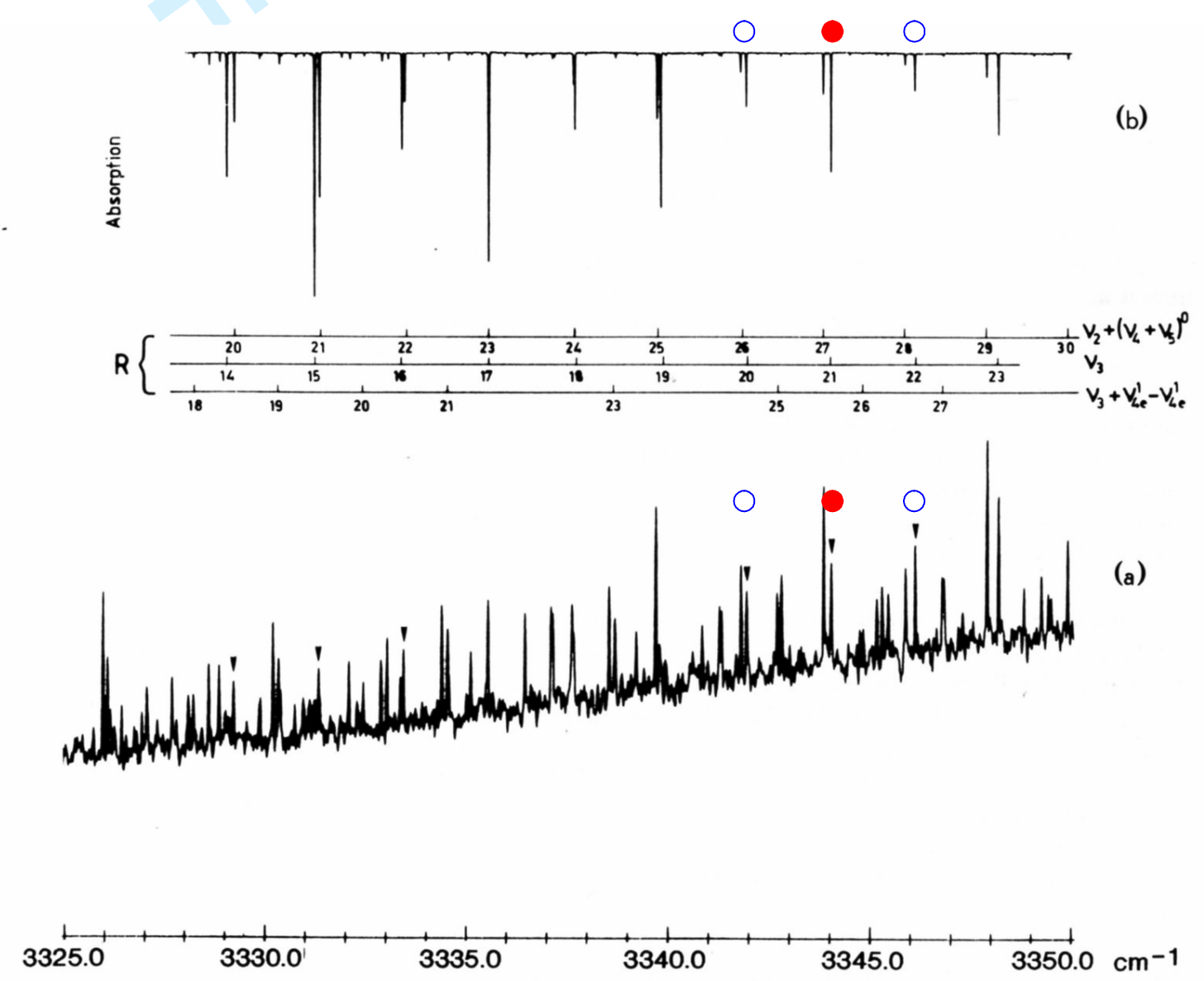
Figure 3: Normal modes of vibration in acetylene. The harmonic frequencies $\left(\tilde{\omega}_{i}^{0}\right)$ are from El Idrissi et al., 1999 [24] $\left({ }^{12} \mathrm{C}_{2} \mathrm{H}_{2}\right)$; Herman et al., 1998 [25] $\left({ }^{12} \mathrm{C}_{2} \mathrm{D}_{2}\right)$; Di Lonardo et al., 1999 [26] $\left({ }^{13} \mathrm{C}_{2} \mathrm{H}_{2}\right)$; Herman et al., $2004[27]\left({ }^{12} \mathrm{C}_{2} \mathrm{HD}\right)$; and Fayt et al., $2007[28]\left({ }^{12} \mathrm{C}^{13} \mathrm{CH}_{2}\right)$.

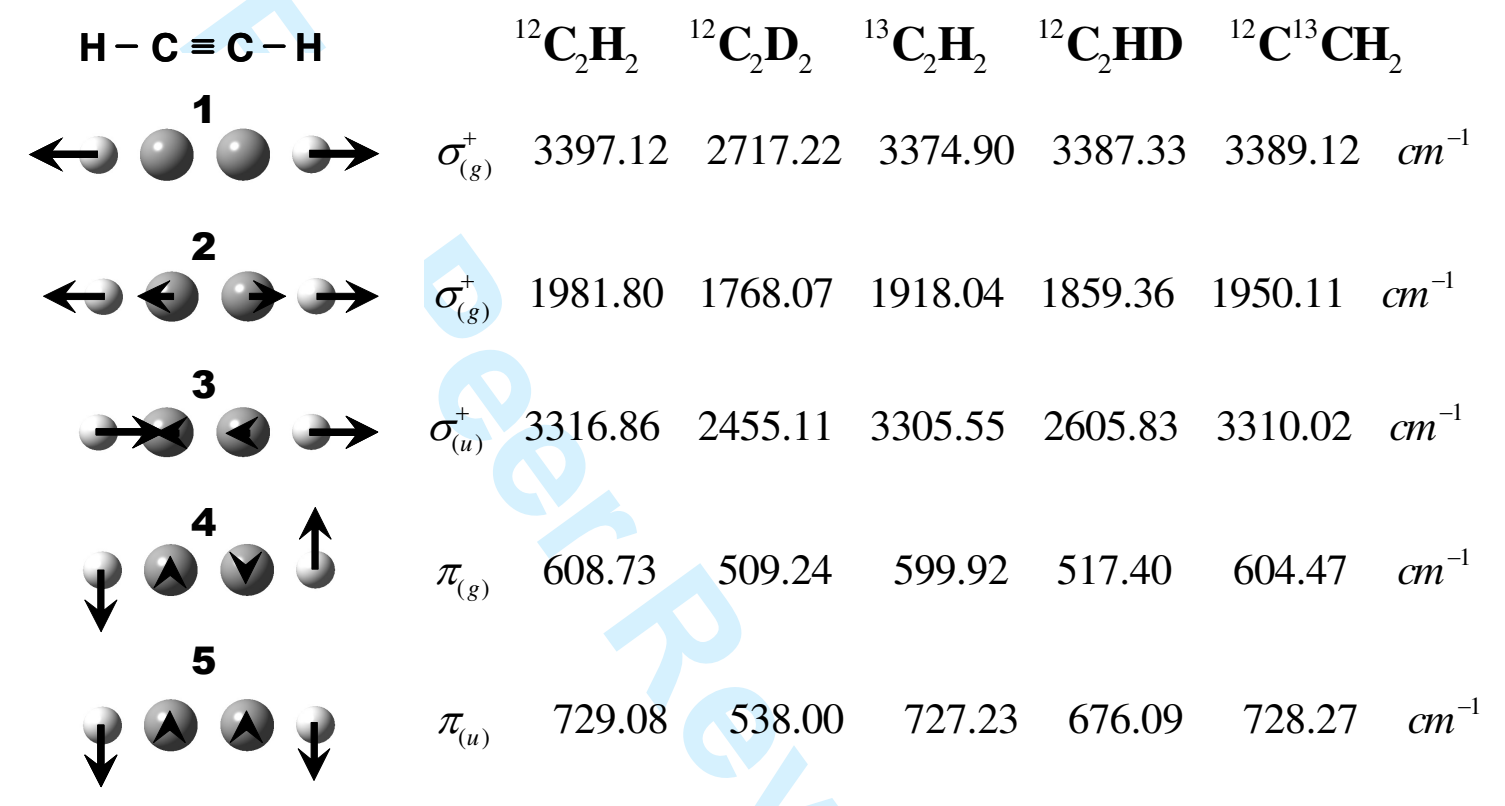


Figure 4: Role of the $g_{45}$ and $r_{45}$ interaction constants, exemplified on the case of the $v_{4}=v_{5}=1$ vibrational state in acetylene. Contribution from terms involving $\mathrm{g}_{44}$ and $\mathrm{g}_{55}$ are identical for both $k$ sublevels and are not accounted for in the energy scheme.

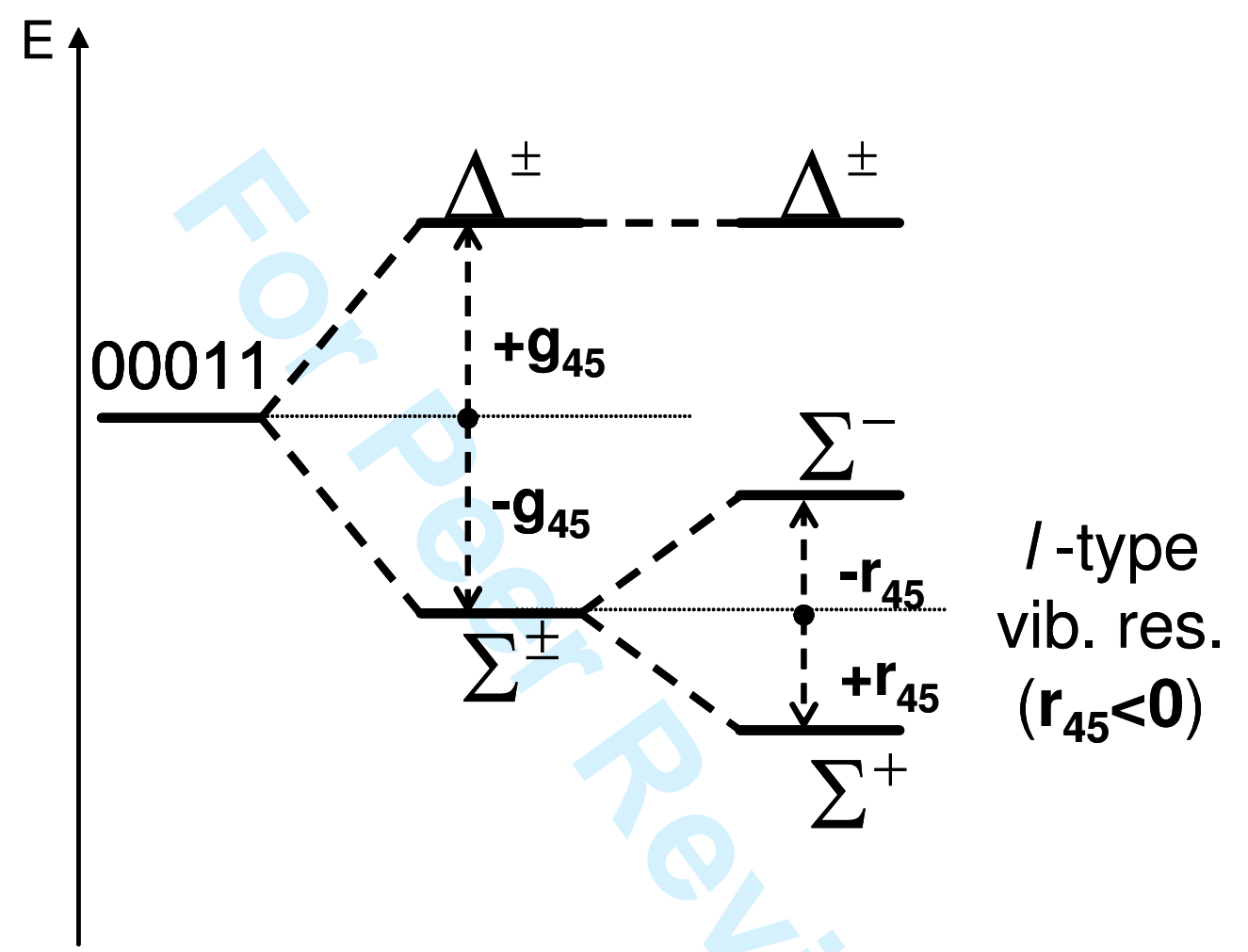


Figure 5: Top: Integrated number of states, $N(E)$ in ${ }^{12} \mathrm{C}_{2} \mathrm{H}_{2}$, in function of the vibrational energy up to $15000 \mathrm{~cm}^{-1}$. The number was calculated from the energies predicted using the extended set of vibrational constants from [24], with each substate with $|\boldsymbol{k}| \neq \mathbf{0}$ counted for 2 in the sum, as explained in [30]. The origin of the energy scale (E) is at the bottom of the well; Bottom: Related density of vibrational states. The origin of the energy is now the GS.
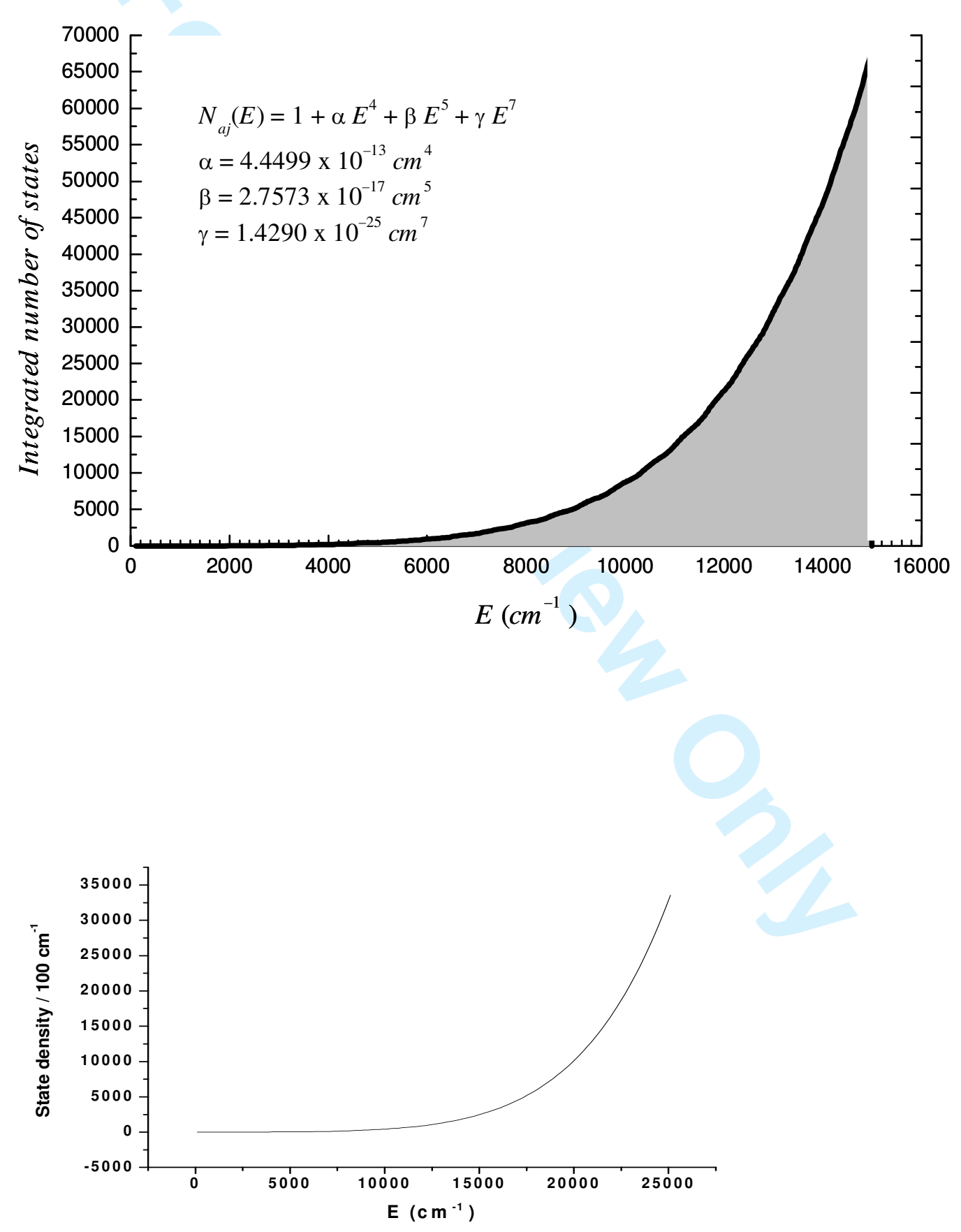
Figure 6: Top: Variation of the absolute value of the electric dipole transition moment $\left(\left|\mathrm{R}_{v}\right|\right)$ upon excitation along the $n v_{1}(\mathrm{CH})$ overtone series in ${ }^{12} \mathrm{C}_{2} \mathrm{HD}$ (adapted from [33]). Triangles refer to measured transition moments and squares to the sum of contributions from the bright and related dark transitions; Bottom: Contribution to the transition moment of the $3 v_{1}$ and $4 v_{1}$ bands in ${ }^{12} \mathrm{C}_{2} \mathrm{HD}$ of the various orders in the electric dipole development, accounting for mechanical anharmonicity in the description of the wavefunctions (adapted from [34]).
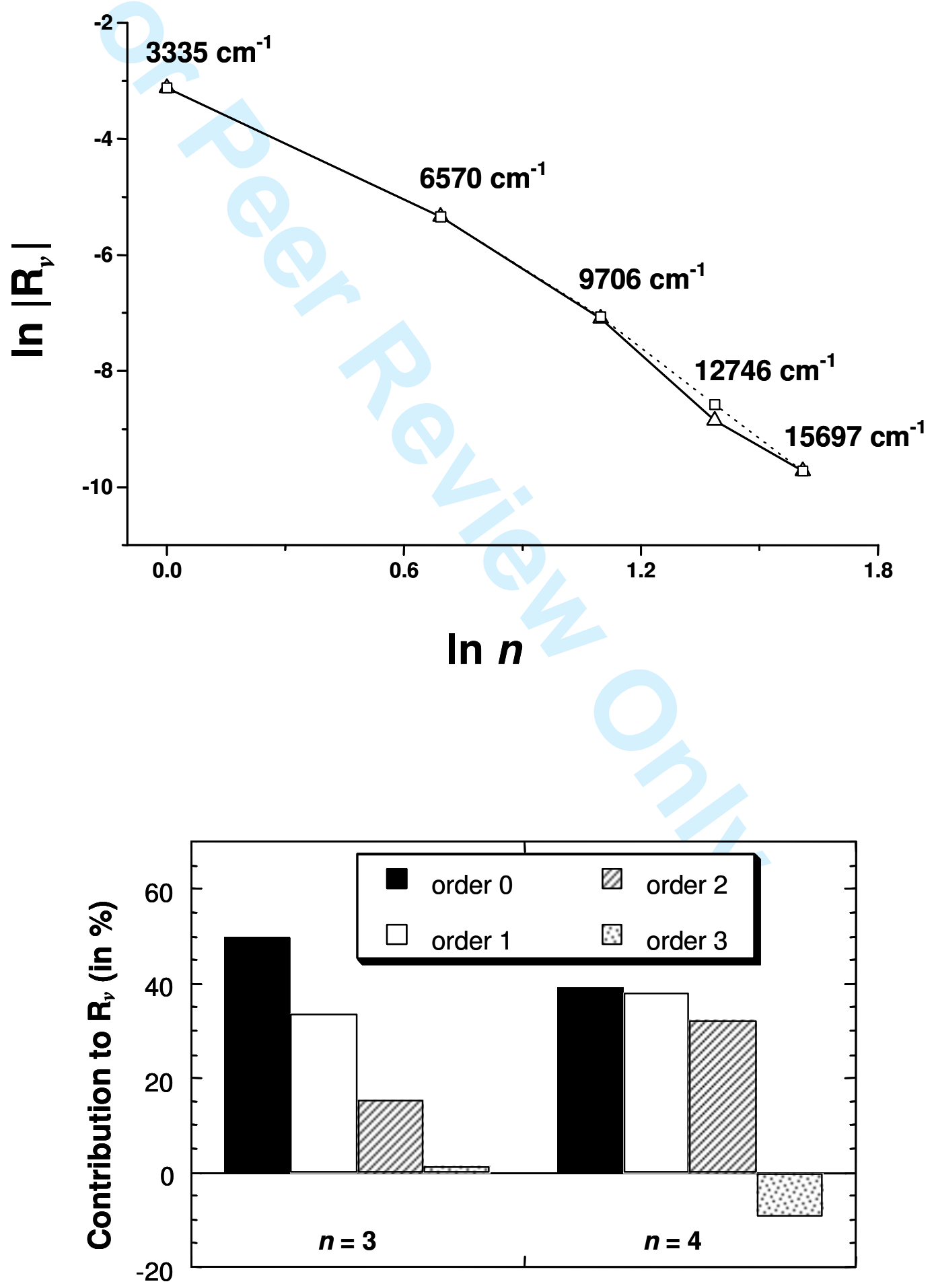

URL: http://mc.manuscripyertone transitipn 
Figure 7: Vibration-rotation absorption FT spectrum of ${ }^{12} \mathrm{C}_{2} \mathrm{H}_{2}$ in the visible range showing the $4^{\text {th }} \mathrm{CH}$ overtone band (adapted from [35]). $\left(\mathrm{P}_{\text {acet }}=\right.$ 250 mbar; $1=49.2 \mathrm{~m}, 6900$ scans co-added).

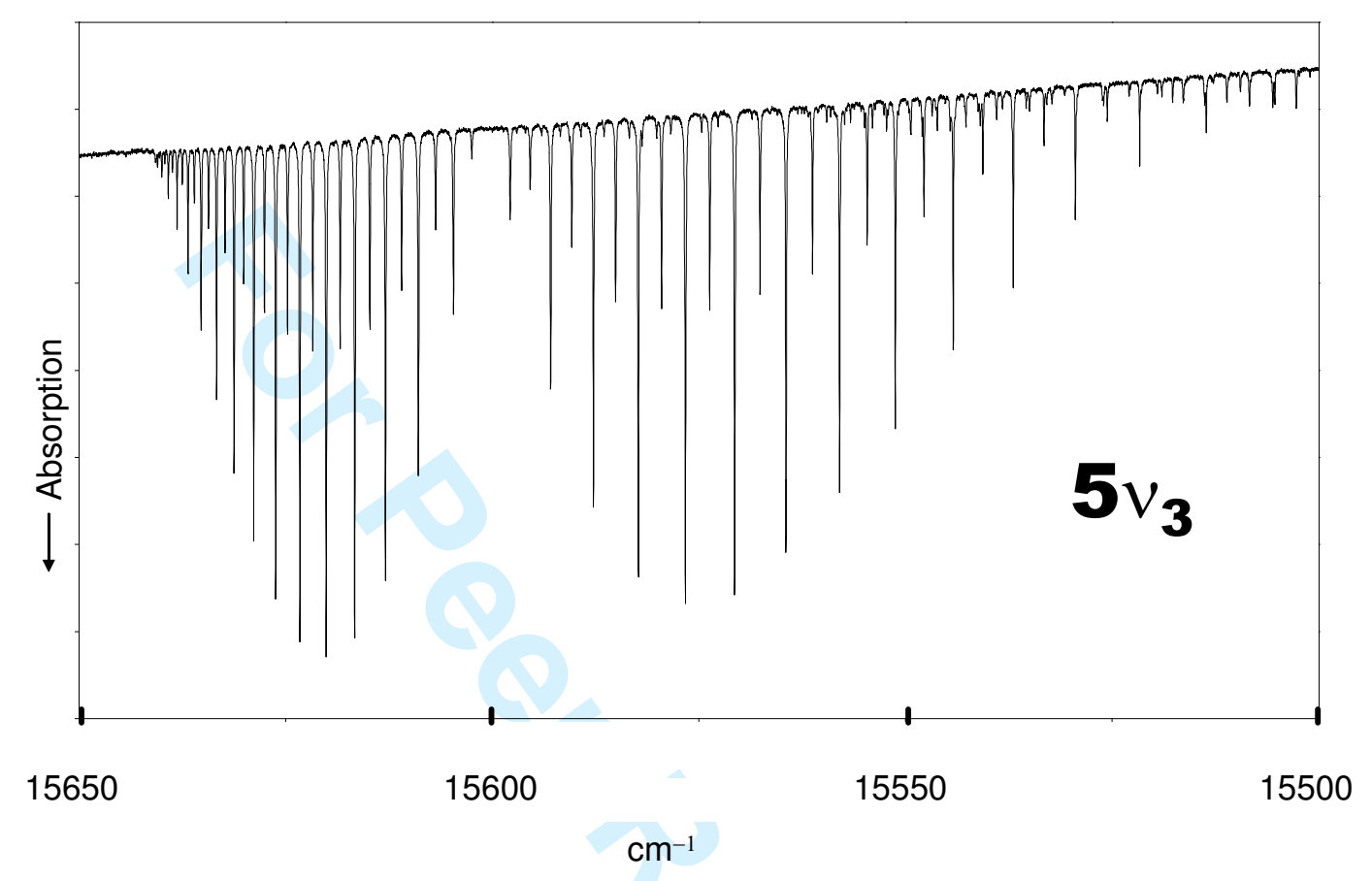


Figure 8 (IN COLOUR): Statistical and dynamical role of the bending vibrations in ${ }^{12} \mathrm{C}_{2} \mathrm{D}_{2}$ (adapted from [43]): Top: Integrated number of states (left ordinate) and residue between the predicted and calculated integrated number of states (right ordinate) Bottom: Related Vibrogram (see text) in which the energy (abscise), defined from the bottom of the potential well, runs from -4000 to $+9000 \mathrm{~cm}^{-1}$, from left to right.

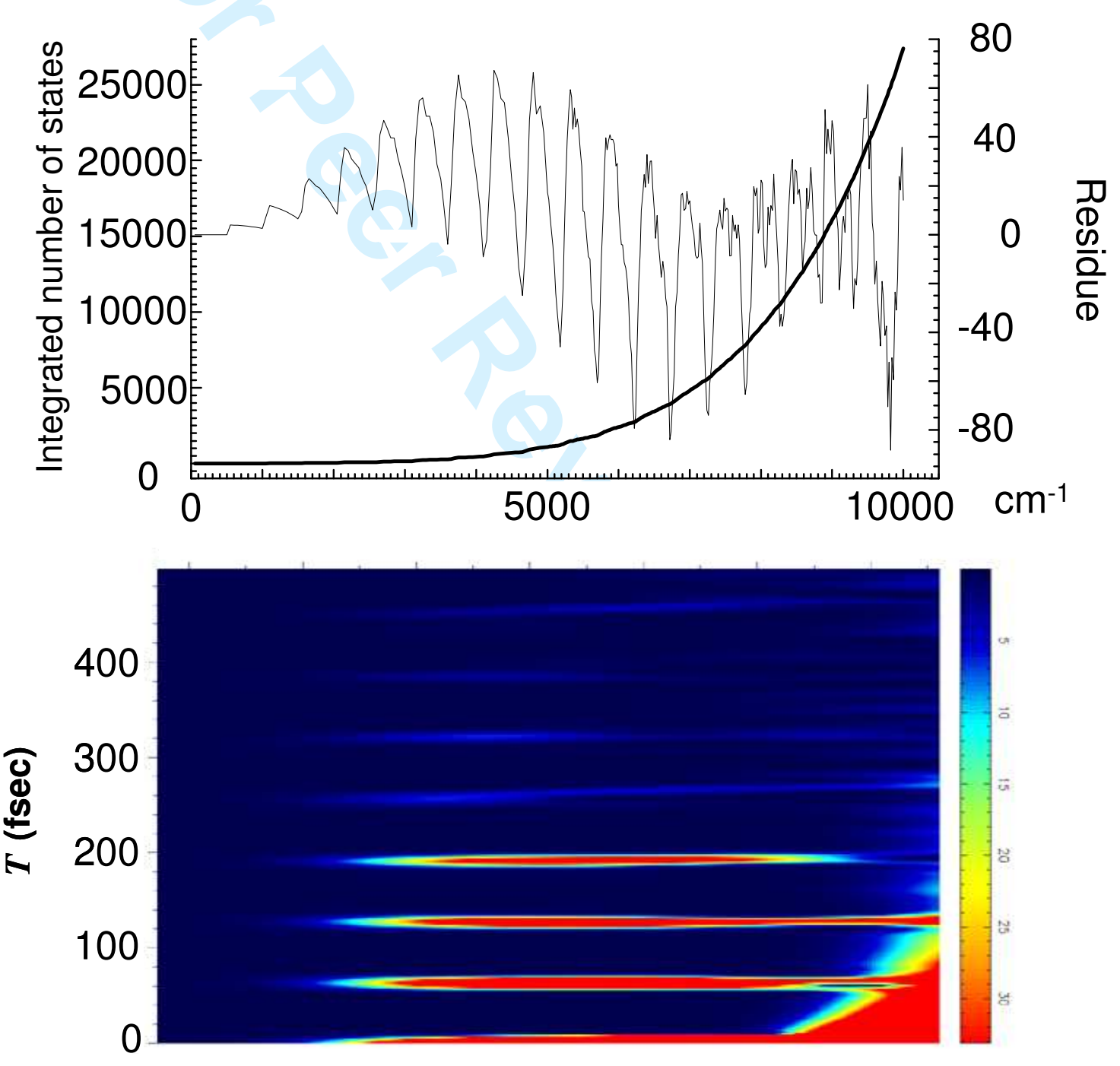


Figure 9: (a) Zero order picture in which two vibrational states with identical energy ( $\Psi_{B}^{0}$ and $\Psi_{D}^{0}$ ) lead to very strong (bright) and very weak (dark) transitions from GS, respectively; (b) a coupling results into two eigenstates $\left(\Psi_{+}\right.$and $\left.\Psi_{-}\right)$with shifted energies and mixed wavefunctions;

(c) the bright character is now equally shared by the two eigenstates. The spectrum, restricted to a single bright transition in the zero order picture (a/bottom) consists in two transitions each with half the initial intensity in the eigen spectrum (c/bottom).

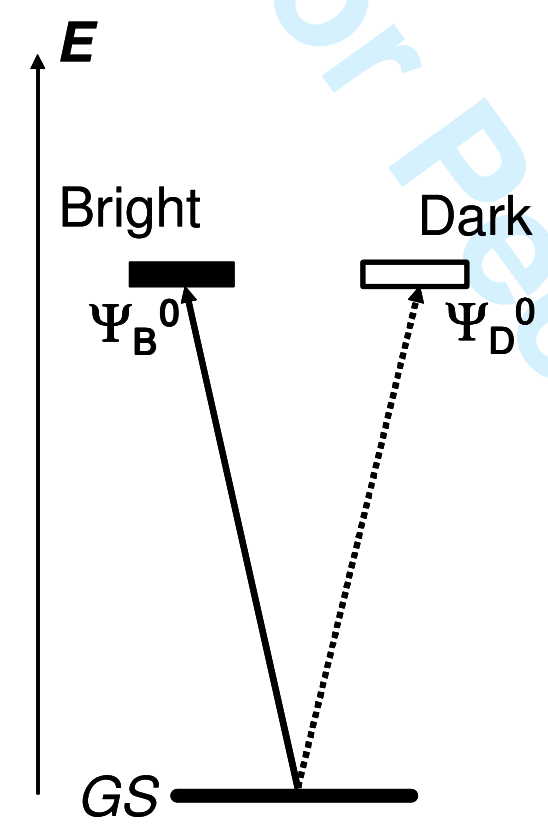

(a)

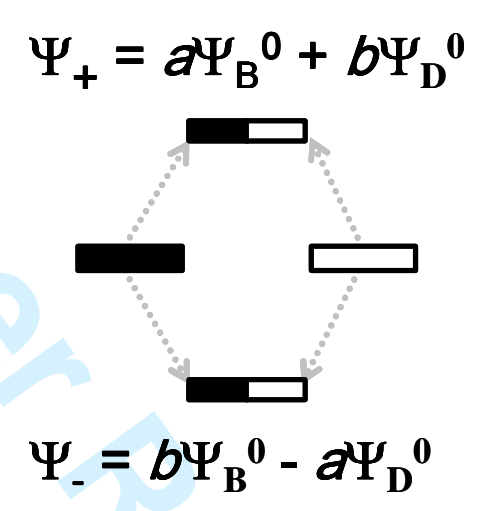

$$
\Psi_{-}=b \Psi_{\mathbf{B}}^{0}-a \Psi_{D^{0}}
$$

(b)

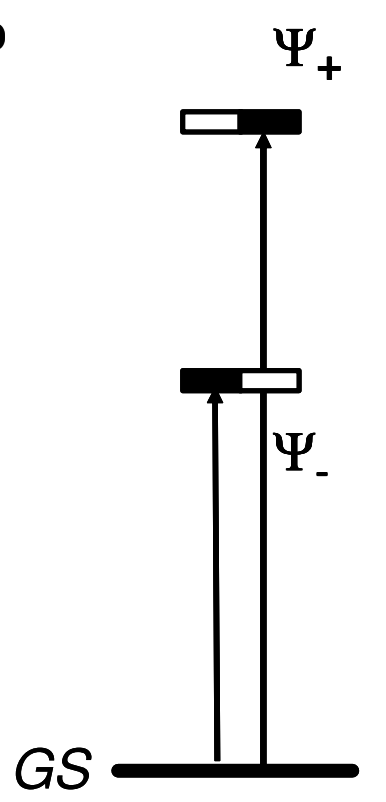

(c)
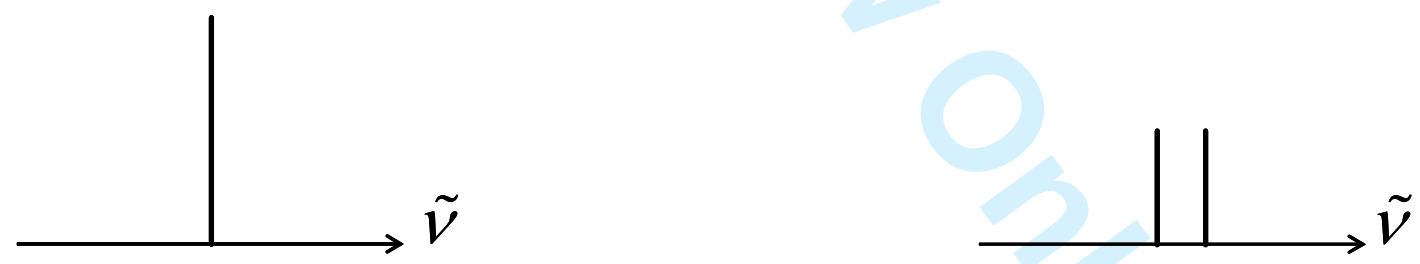
Figure 10: Example of intensity borrowing through the 1/255 anharmonic resonance in ${ }^{12} \mathrm{C}_{2} \mathrm{HD}$ (adapted from [34]). Additional bands from ${ }^{12} \mathrm{C}_{2} \mathrm{H}_{2}$, with typical intensity alternation, are observed around $12700 \mathrm{~cm}^{-1}$ in the bottom spectrum. Zero order dark $Q$ branches in $\Pi-\Sigma^{+}$transitions are identified on the top and middle figures, borrowing their intensity from the zero order $3 v_{1}+v_{4}(k=1)$ and $3 v_{1}+v_{5}(k=1)$ bright transitions, respectively. A zero order $R$ branch borrowing intensity from the $4 v_{1}$ $\Sigma^{+}-\Sigma^{+}$bright transition is indicated in the bottom spectrum.
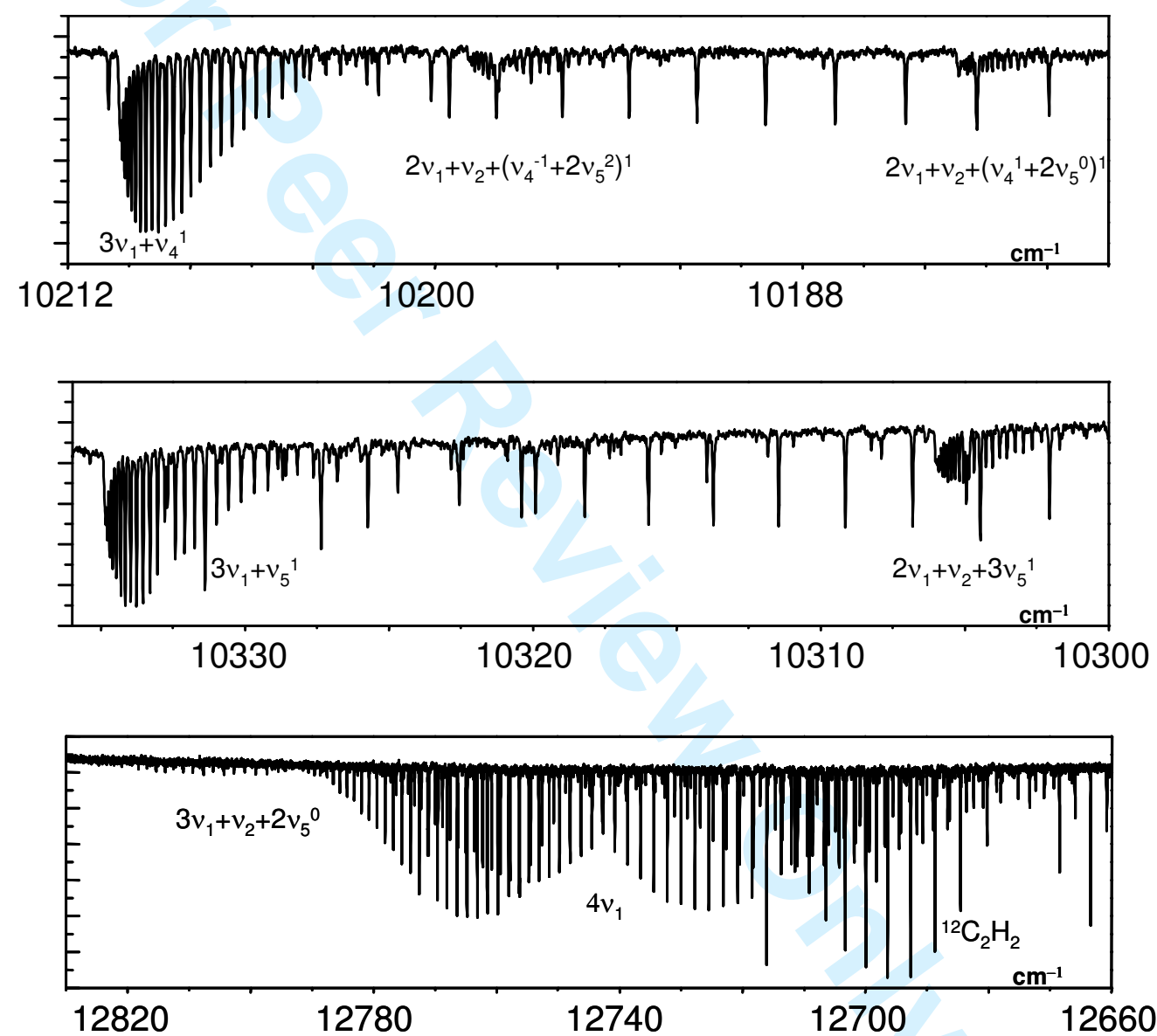
Figure 11: Energy difference $\left(\Delta \mathrm{E}=\mathrm{E}\left(v_{1}, v_{2}, v_{3}, v_{4}, v_{5}\right)-\mathrm{E}\left(v_{1}-1, v_{2}+1, v_{3}, v_{4}, v_{5}+2\right)\right)$ between some pairs of states interacting through the 1/255 anharmonic resonance in ${ }^{12} \mathrm{C}_{2} \mathrm{HD}$, plotted as a function of the approximate state energy. Only one of the interacting states is identified with the help of the related values of the vibrational quantum numbers, $v_{1}$ to $v_{5}$. The three sets of interaction highlighted in figure 10 are framed.

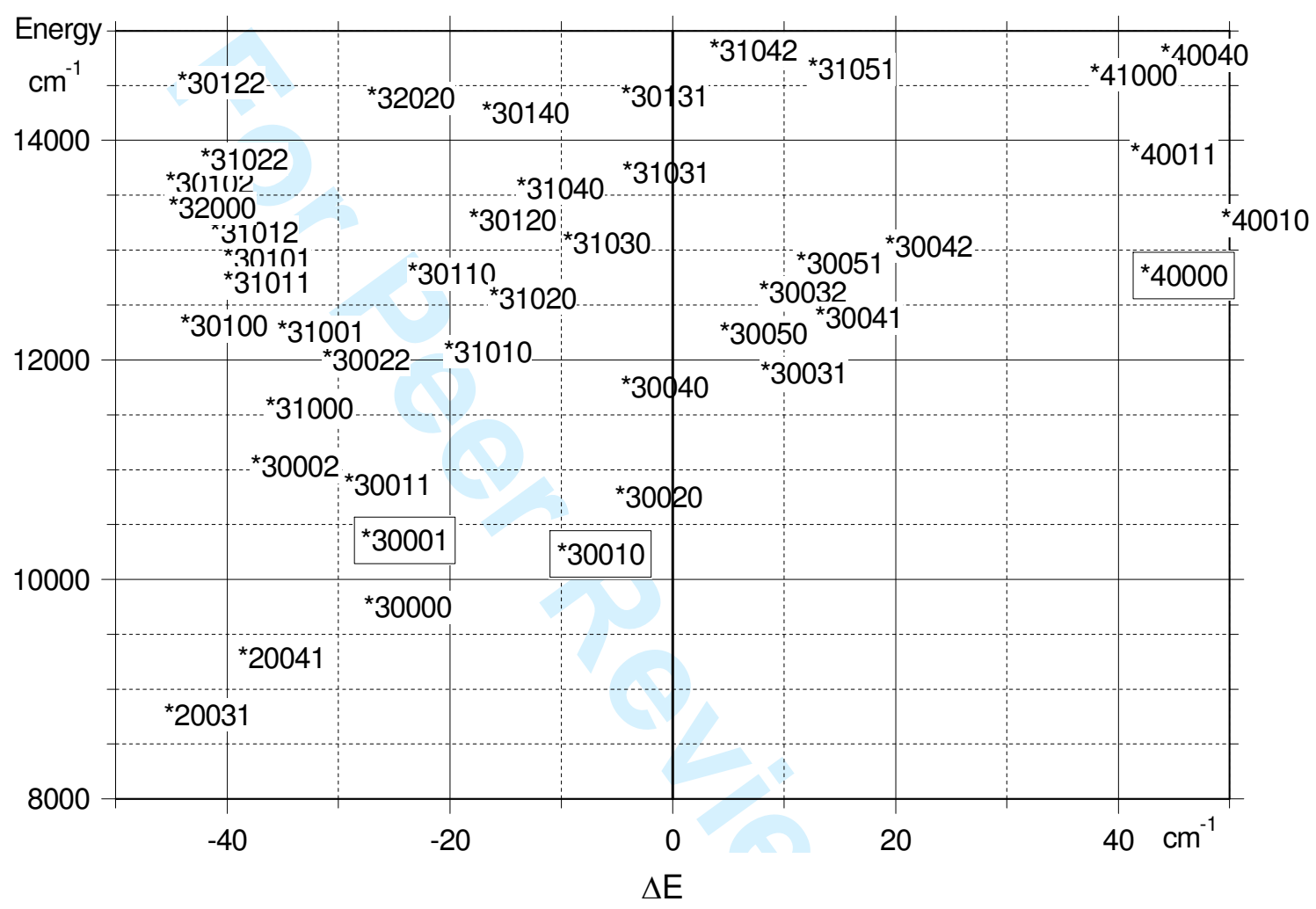


Figure 12: Overtone spectrum of ${ }^{12} \mathrm{C}_{2} \mathrm{HD}$ recorded using 2T-FT-ICLAS (middle), showing a band not observed on the FT spectrum (bottom). The product of absorption pathlength $(14.64 \mathrm{Km})$ and pressure $(120 \mathrm{hPa})$ conditions in the laser experiment is about 500 times larger than in the FT experiment. The line assignment is indicated (middle) and the band structure is simulated (upper) (adapted from [58]).

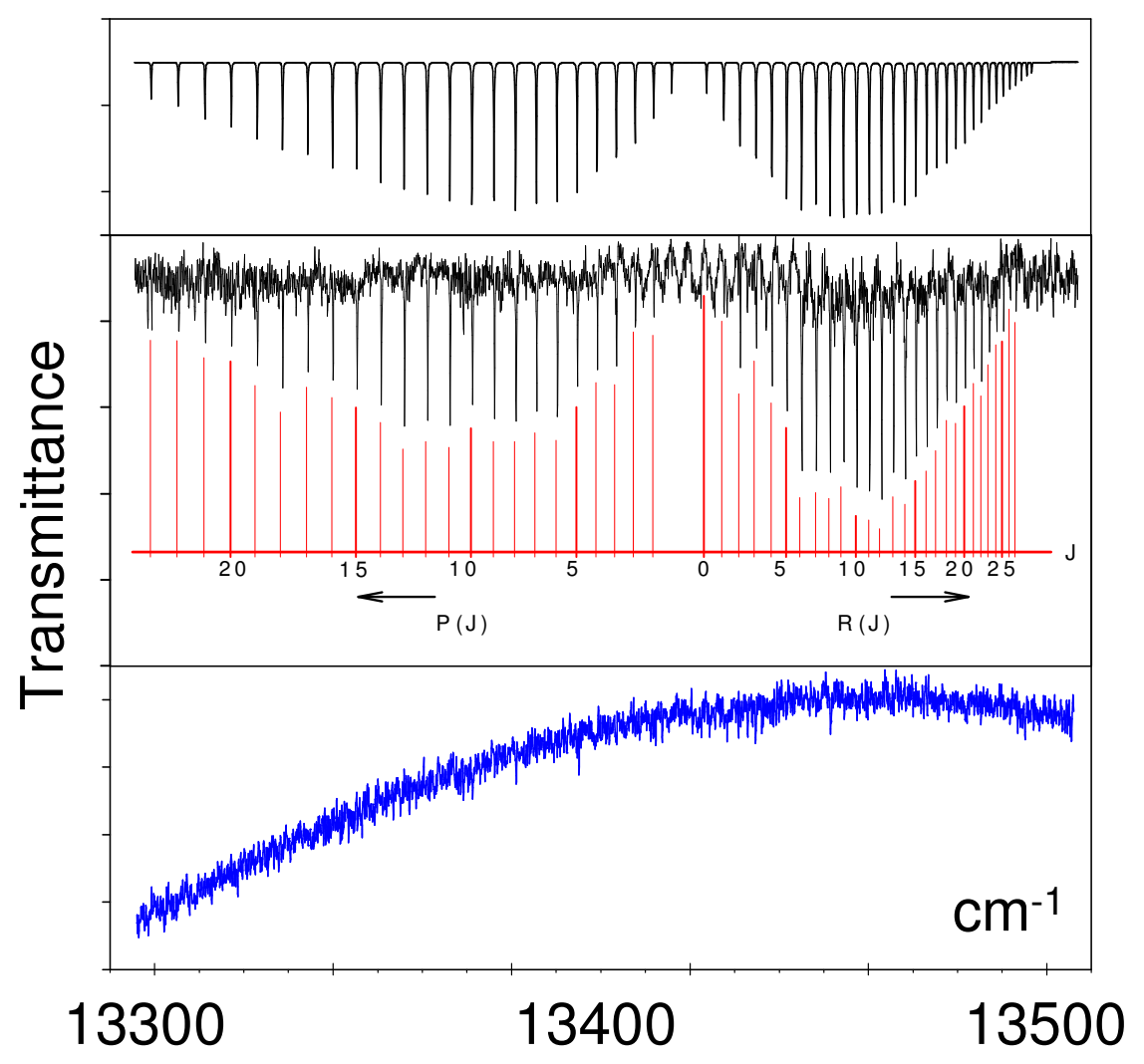


Figure 13: Left: Scheme of bright/dark multiple intensity borrowing in a straight absorption scheme; and, Right: dispersed fluorescence spectrum of ${ }^{12} \mathrm{C}_{2} \mathrm{H}_{2}$ ranging over some $600 \mathrm{~cm}^{-1}$, observed (left) and simulated (right), adapted from [66]. Each straight line in the simulation indicates, with appropriate relative intensity, a predicted fractionation of the zero order bright transition.

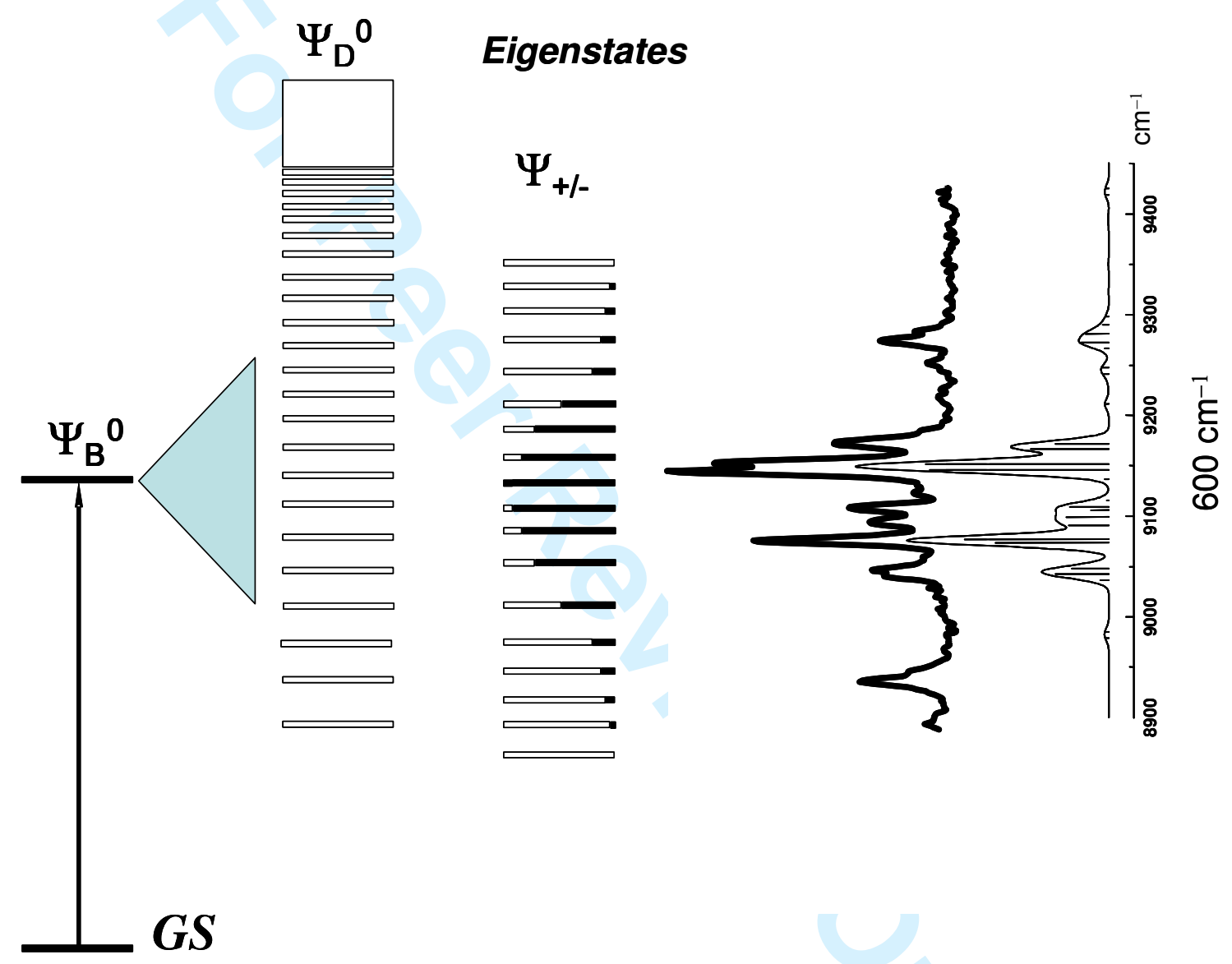


Figure 14: Scheme of the $\left\{N_{s}, N_{r}, k,+, u\right\}=\{2,10,0,+, u\}$ cluster in ${ }^{12} \mathrm{C}_{2} \mathrm{H}_{2}$, with anharmonic resonance connecting $\left(v_{1} v_{2} v_{3} v_{4}^{l_{4}} v_{5}^{l_{5}}\right), \Sigma^{+}$vibrational states identified (from [68]).

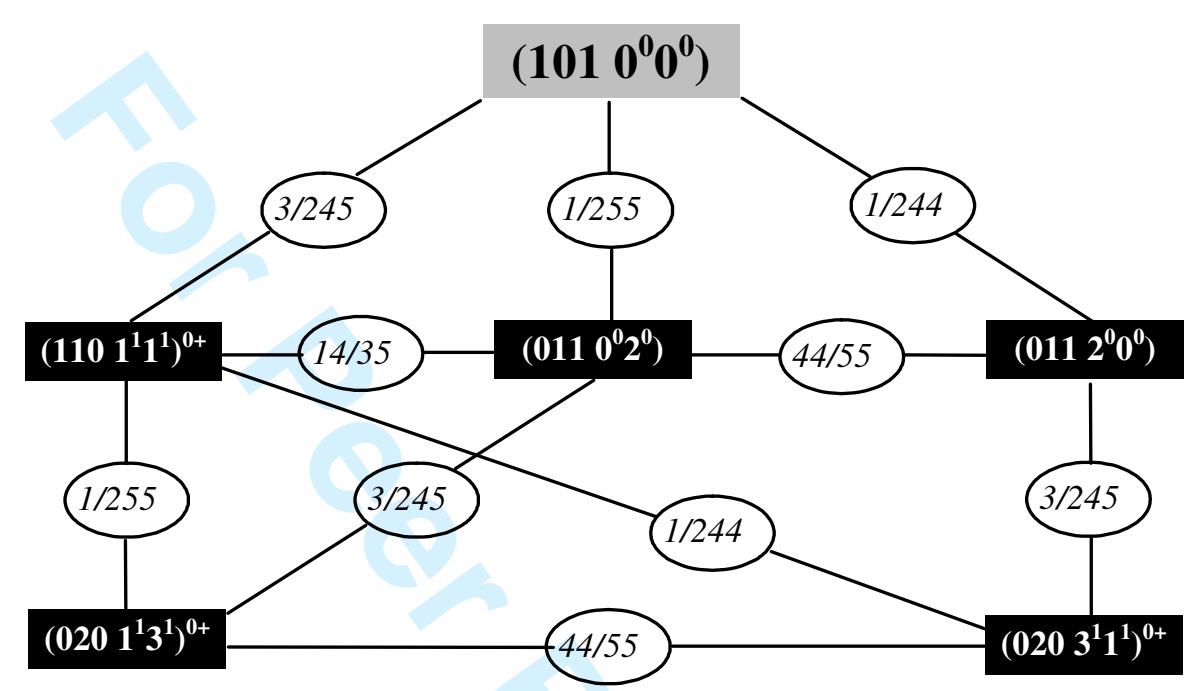


Figure 15: Evolution with the vibrational energy of the $\left\{N_{s}, N_{r}\right\}$ clustering of the states, in ${ }^{12} \mathrm{C}_{2} \mathrm{H}_{2}$. The average energies of the so-called sub-polyad $\tilde{v}^{a v}\left(N_{r}, N_{s}\right)$ are taken with respect to each average polyad energy $\tilde{v}^{a v}\left(N_{r}\right)$ (adapted from [30]).

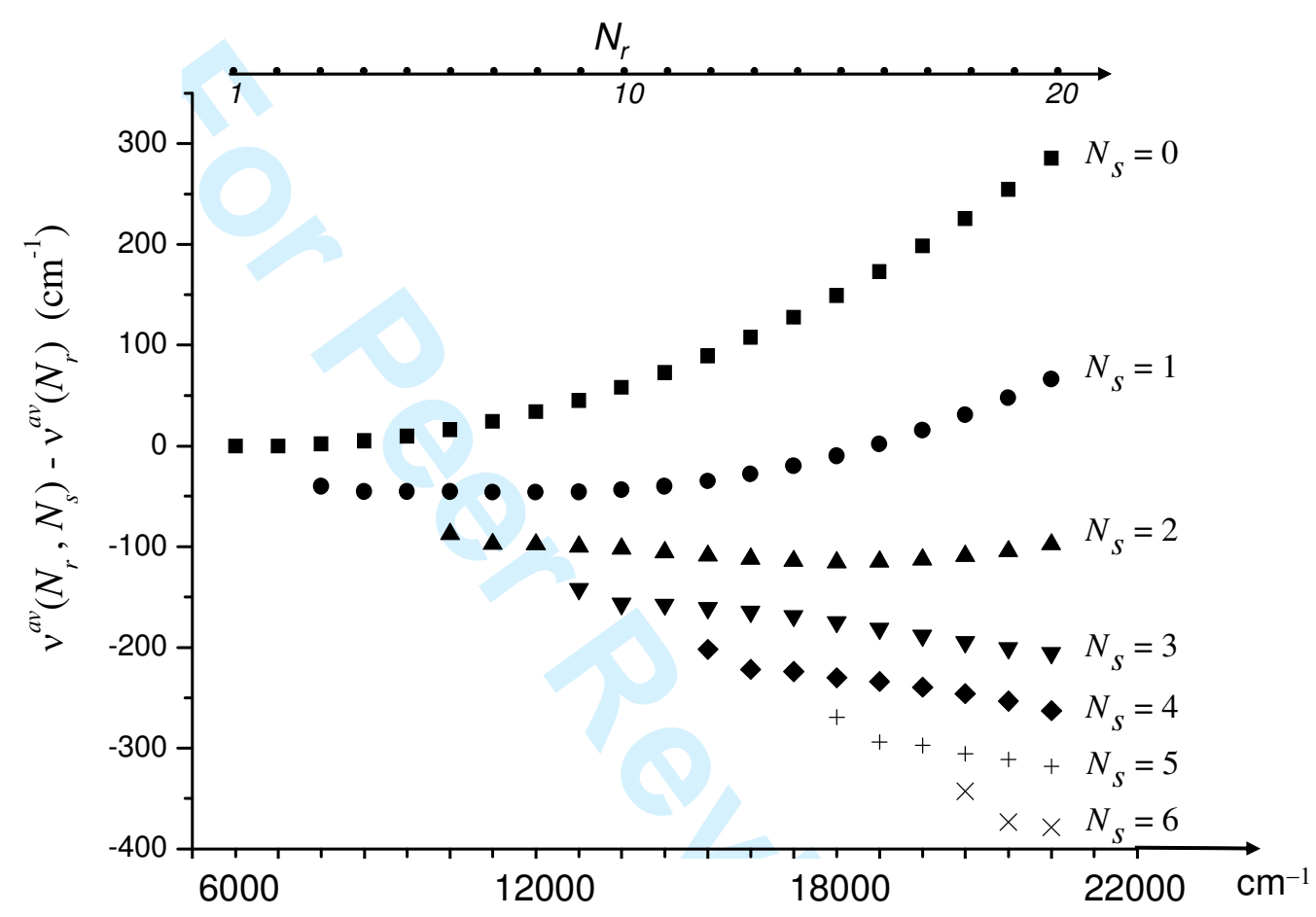


Figure 16: Portions of the FT-transmittance spectrum of ${ }^{12} \mathrm{C}^{13} \mathrm{CH}_{2}$ recorded at ULB (adapted from [73]). The sample pressure was 5.05 mbar, the temperature $273 \mathrm{~K}$, and the absorption pathlength $55.1 \mathrm{~m}$. The upper and middle spectra range from about 5700 to 10000 and 5700 to $6000 \mathrm{~cm}^{-1}$, respectively.

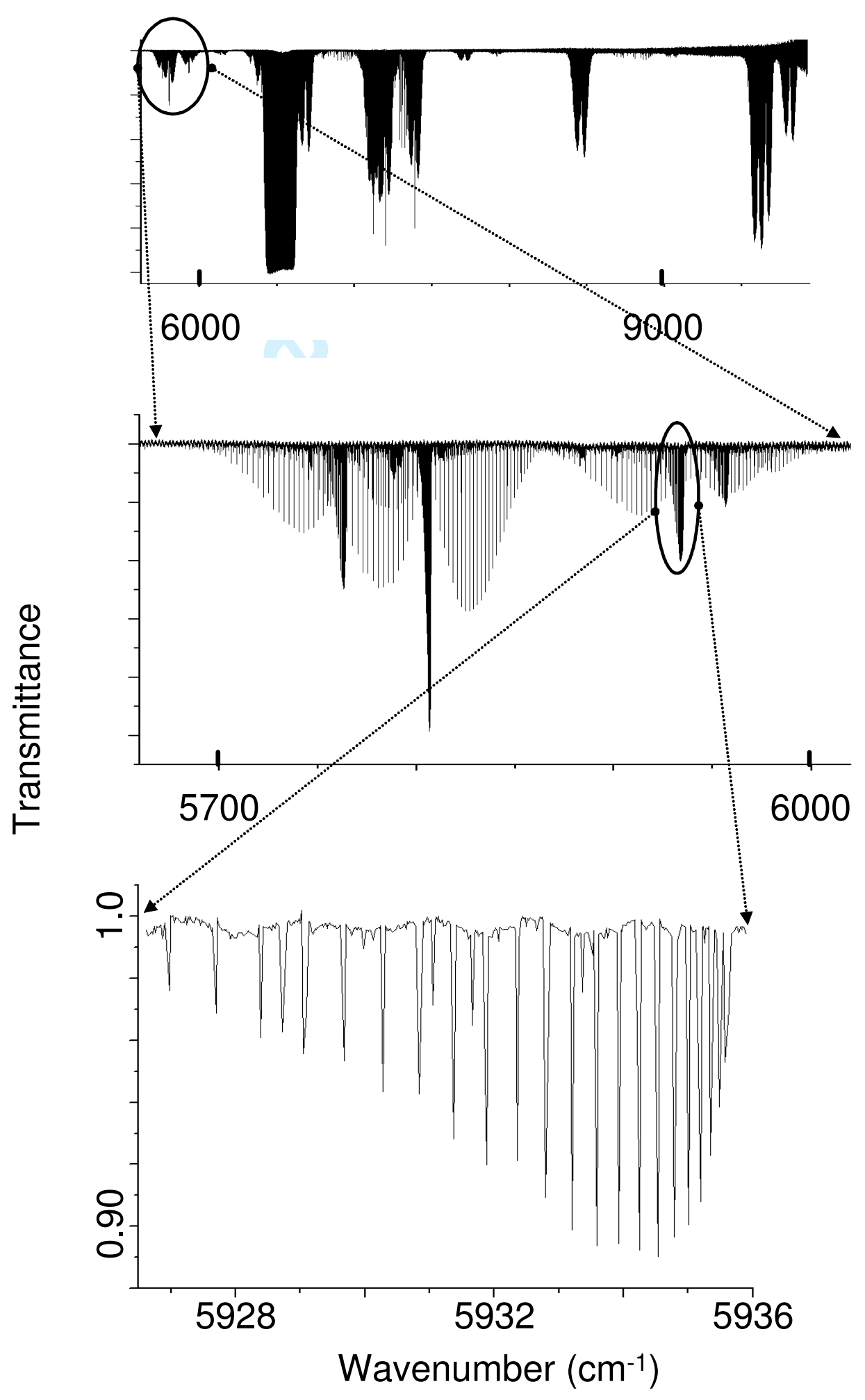


Figure 17: Summary of the procedure to define $e / f$ rotation-vibration states in linear species, as defined in [80], applied to the $v_{4}=1, l_{4}=1$ state in acetylene. In this specific case, $e$ levels are below $f$-ones $\left(\mathrm{q}_{4}<0\right)$.

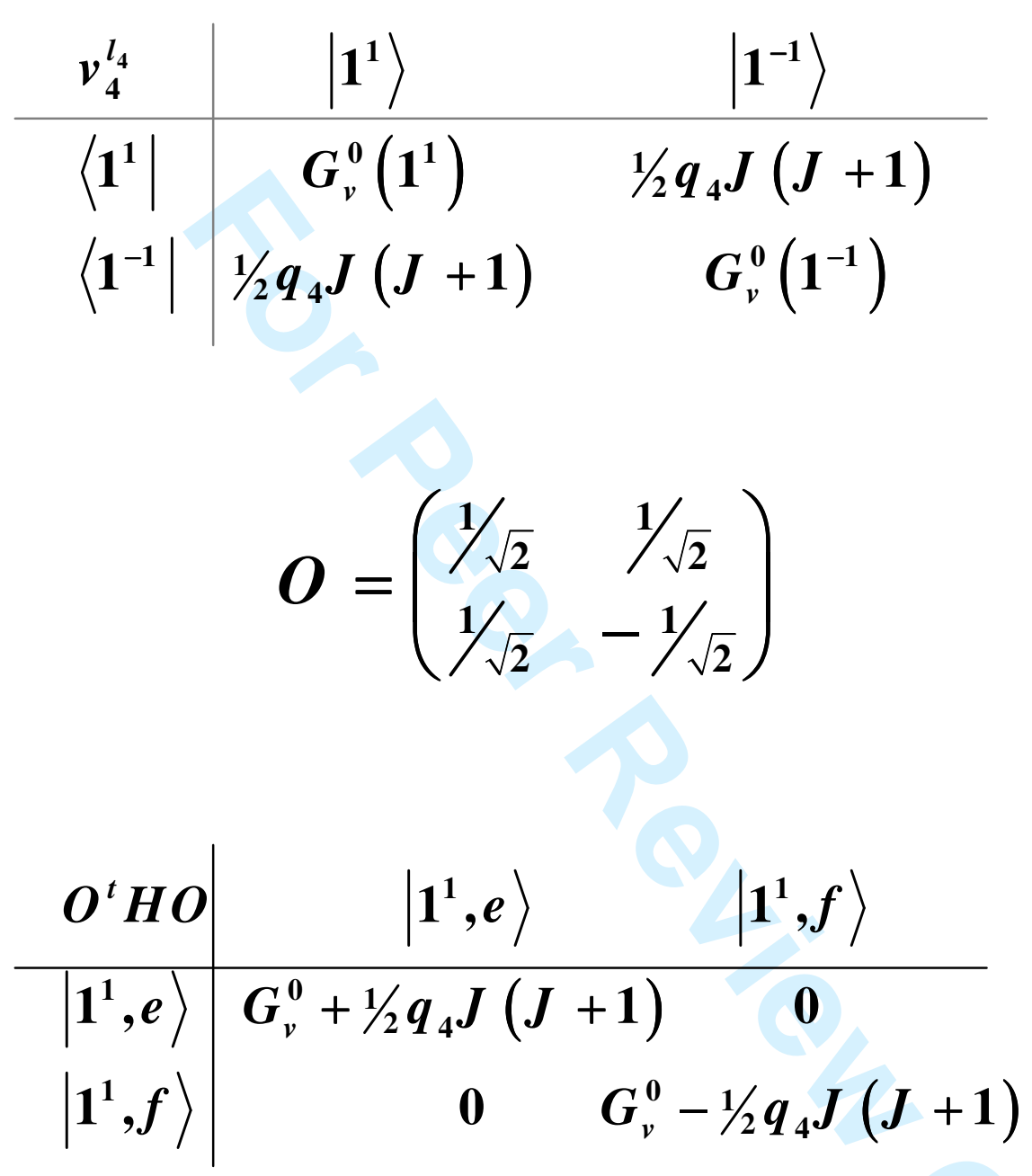


Figure 18: Detailed rotational structure, including the role of nuclear spin statistics, for all $\Sigma-\Pi$ transition-types in ${ }^{12} \mathrm{C}_{2} \mathrm{H}_{2}$ (adapted from [10]). Ortho/Para components are in full/dotted lines.
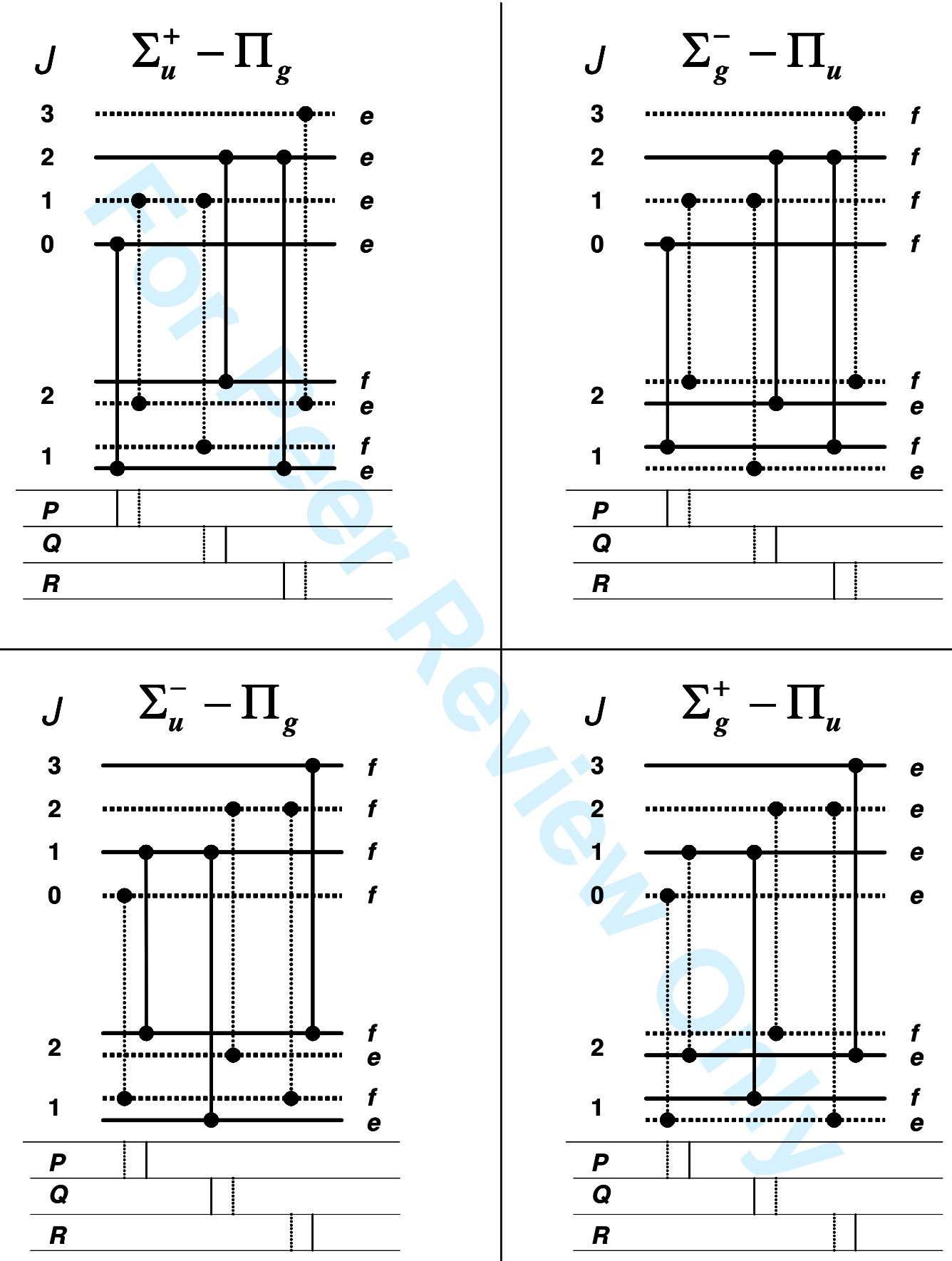
Figure 19: Sequence of couplings, with main related parameters highlighted at the bottom, affecting the $v_{3}=1$ and $v_{2}=v_{4}=v_{5}=1$ vibrational states (left) and related reduced rotational energies (as defined in Eq. (3.5) ) (right), in ${ }^{12} \mathrm{C}_{2} \mathrm{H}_{2}$. All states highlighted are of $u$ symmetry. Other states appear on the reduced energy graph, whose assignment is not given.

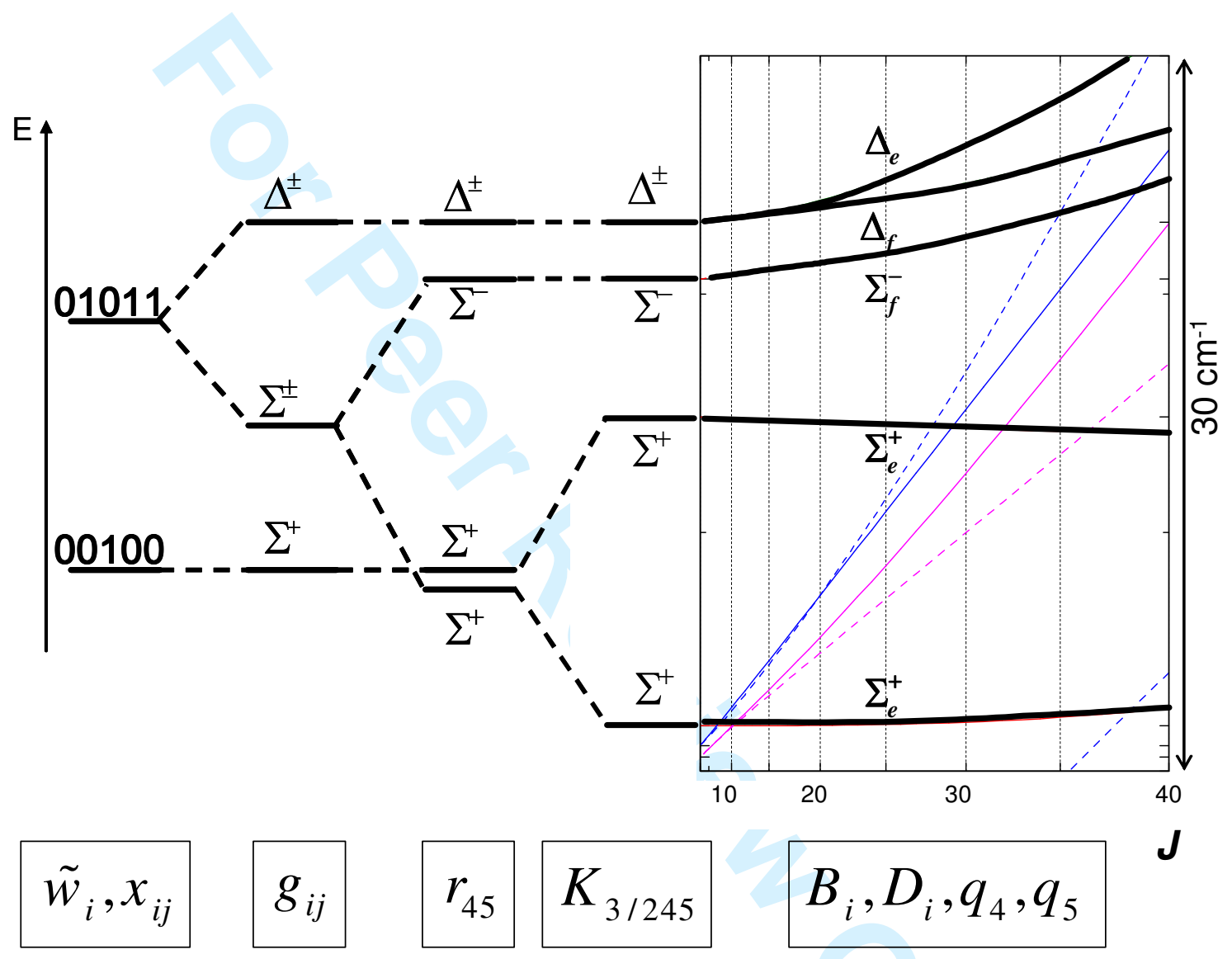


Figure 20: Eigenvector coefficients for the three interacting $\left(v_{1} v_{2} v_{3} v_{4} v_{5}, l_{4} l_{5}\right)$ zero order states in ${ }^{12} \mathrm{C}_{2} \mathrm{H}_{2}$ identified in the graphs and pictured in figure 19. The labels $\bullet, \bullet$ and $\nabla$ refer to the $(01011,11)$; $(00100,00)$; and $(01011,1-$ 1) zero order states, respectively (calculated from [82])

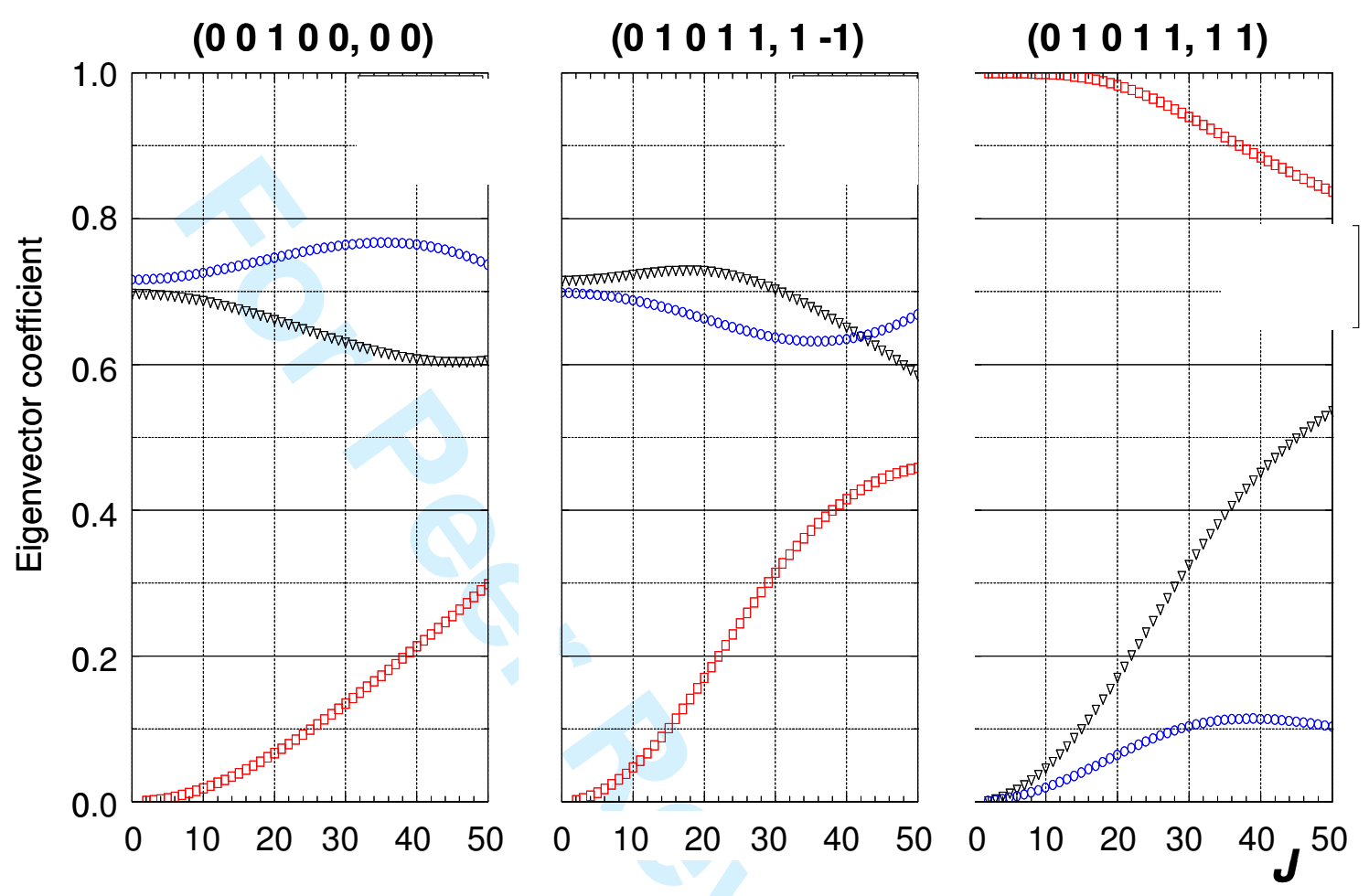


Figure 21: Simulated portion of the infrared spectrum of ${ }^{12} \mathrm{C}_{2} \mathrm{H}_{2}$ demonstrating the role of the various coupling schemes around $v_{3}$ in making a zero order forbidden $\Delta-\Sigma^{+}$transition emerge, actually at higher $J$-values, identified through stick lines drawn broader on the simulation (adapted from [82]).

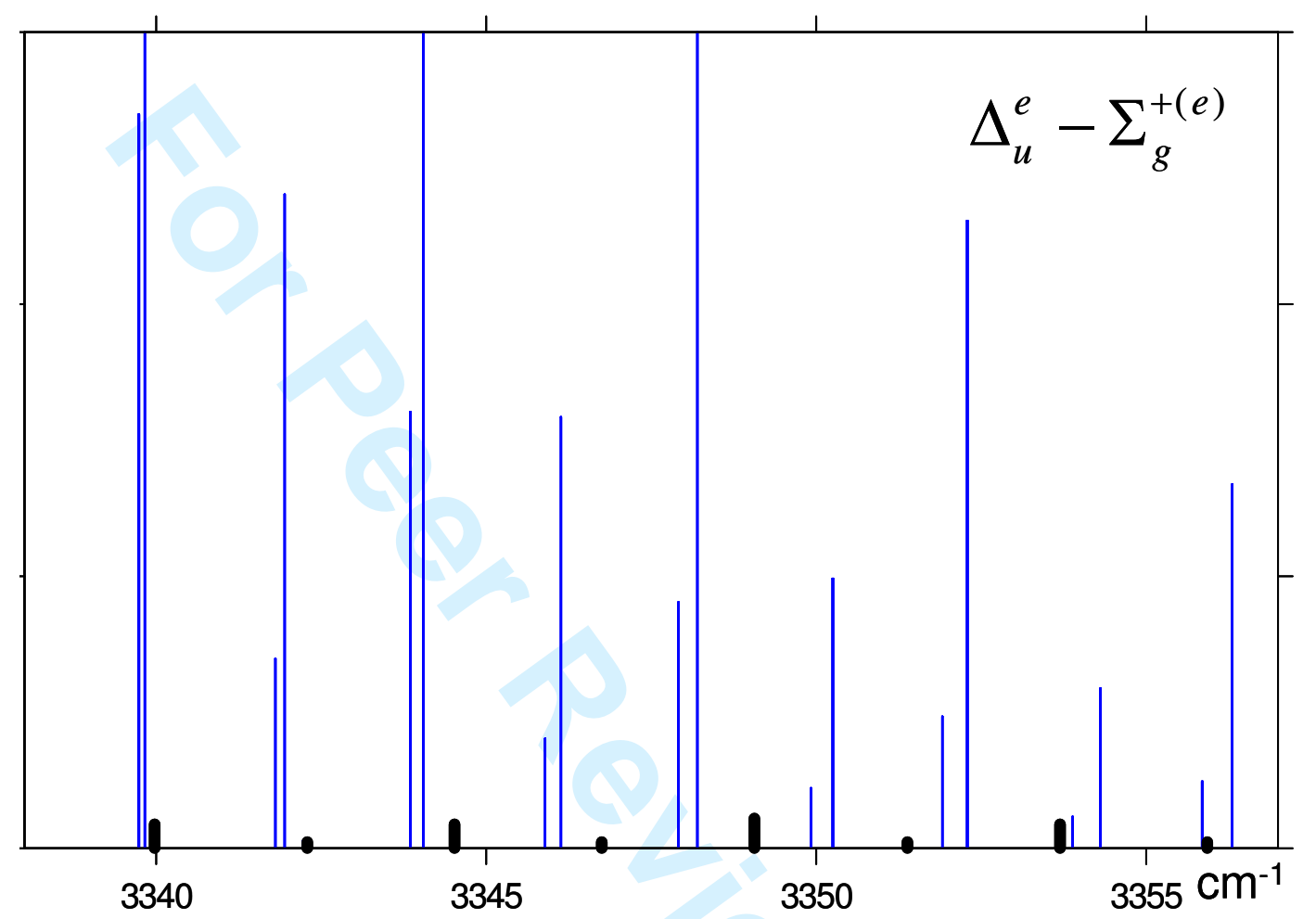


Figure 22: Reduced energies, defined as in Eq. (3.5), as a function of $J$ for the observed vibration-rotation levels of the $\left(v_{1} v_{2} v_{3} v_{4}^{l_{4}} v_{5}^{l_{5}}\right) \equiv\left(0030^{0} 0^{0}\right)\left(\Sigma^{+}\right)(\mathbf{\bullet})$, $\left(0213^{1} 1^{1}\right)(\circ)$ and $\left(0303^{3} 3^{-1}\right)(\Delta)(\Delta)$ states of ${ }^{13} \mathrm{CH}^{12} \mathrm{CH}$, with all vibrational labels corresponding to zero order assignments (adapted from [74]).

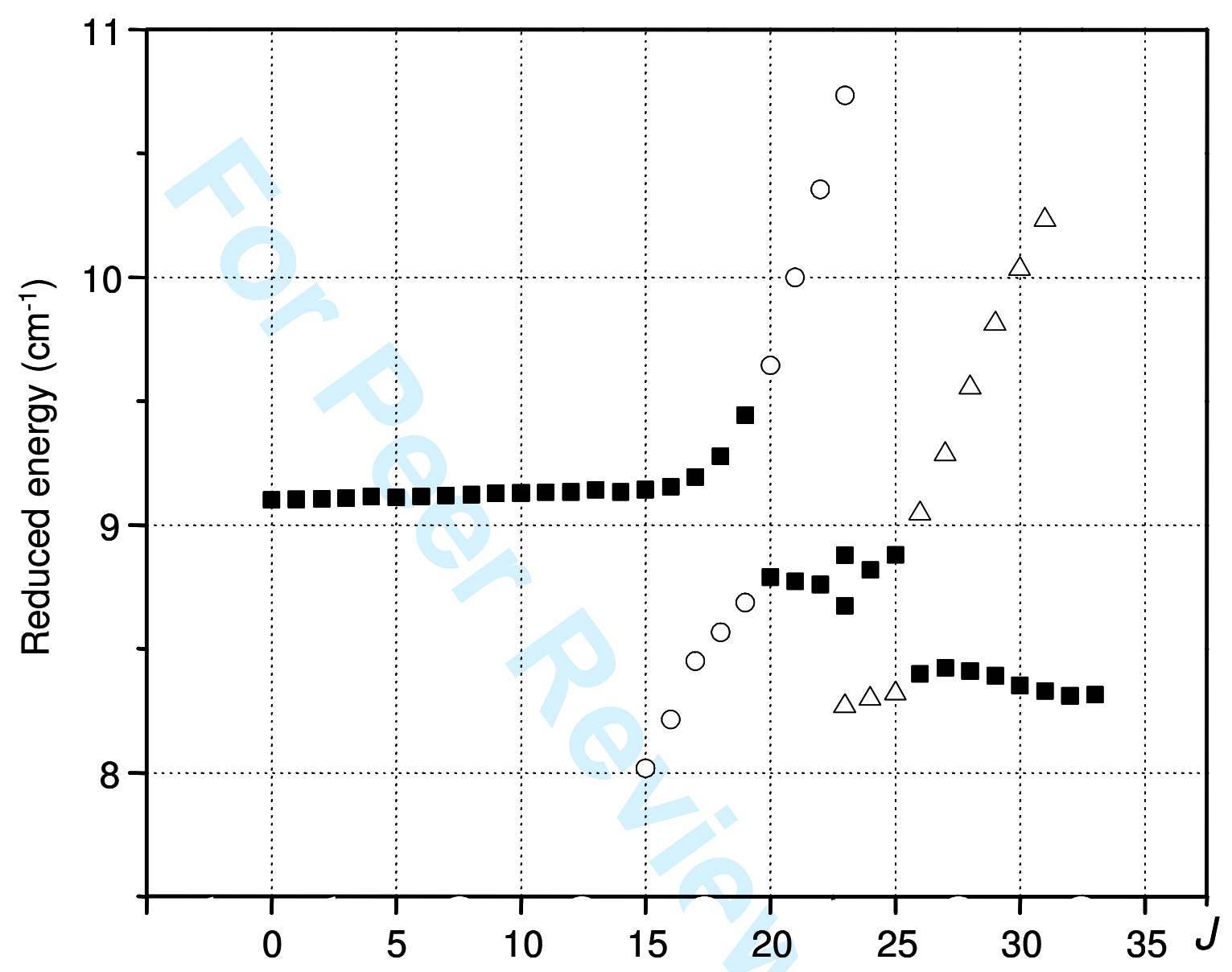


Figure 23: Portion of the $\left\{N_{s}, N_{r}\right\}=\{3,15\}$ cluster in acetylene (adapted from [39]). Arrows show the resonances, identified at the bottom of the figure, connecting $\left(v_{1} v_{2} v_{3} v_{4} v_{5}\right)$ vibrational states. The two sets of states separated by the horizontal dotted line are connected in ${ }^{12} \mathrm{C}^{13} \mathrm{CH}_{2}$ but not in ${ }^{12} \mathrm{C}_{2} \mathrm{H}_{2}$ because of $u / g$ selection rules forbidding the $1 / 245$ and $3 / 244$ couplings to occur.

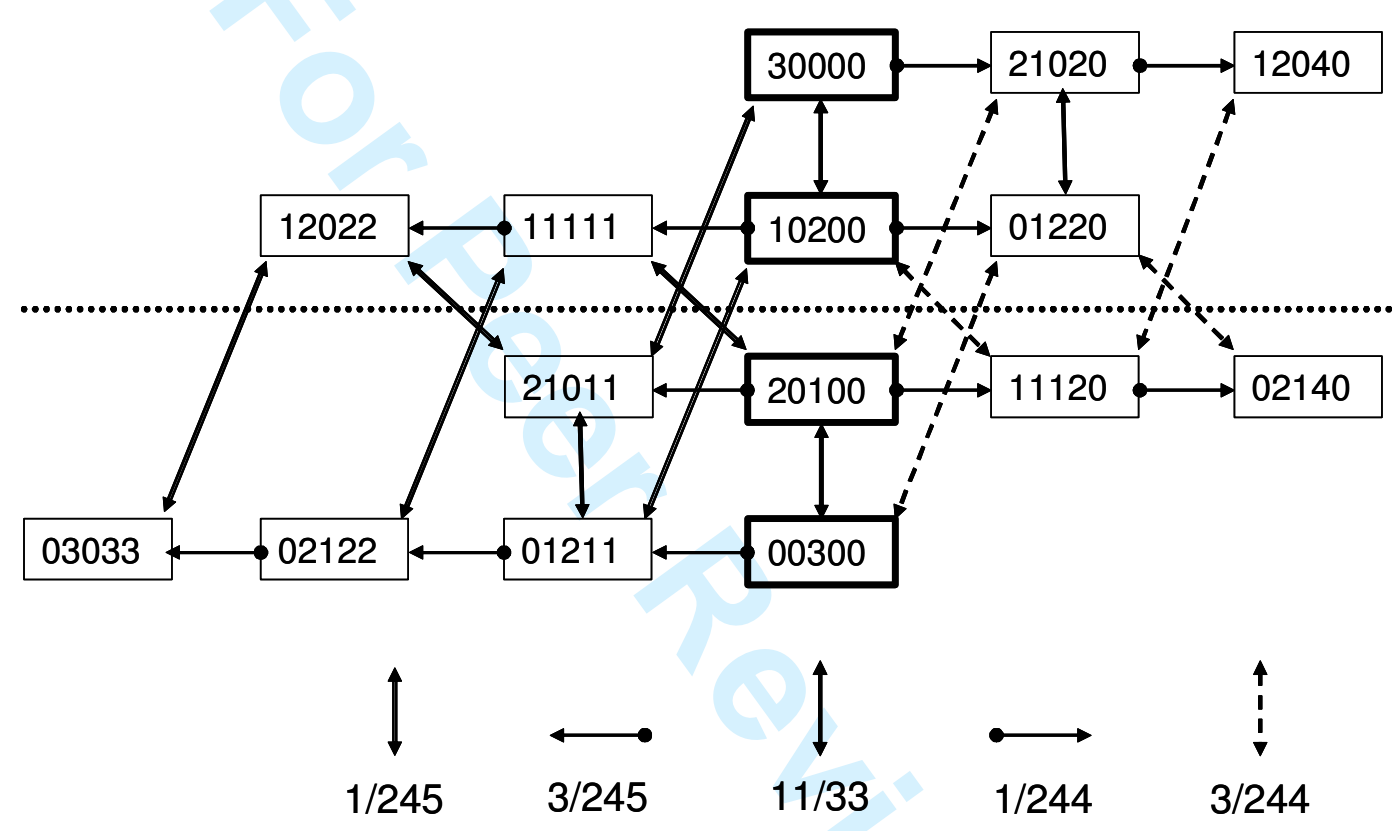

$(g)$ 
Figure 24: Illustration of the $k$-mixing in the global picture of acetylene, from a portion of the $\left\{N_{s}, N_{r}\right\}=\{2,10\}$ vibration-rotation cluster in ${ }^{12} \mathrm{C}^{13} \mathrm{CH}_{2}$, in which higher order interaction terms, with upper/lower signs valid for $e / f$ symmetries are included, all defined in [28]. The symbol $\mathbf{X}$ indicates the absence of coupling term. The matrix is symmetric.

\begin{tabular}{|c|c|c|c|c|c|c|c|c|c|}
\hline$v_{1} v_{2} v_{3} v_{4} v_{5}, l_{4} l_{5}$ & $\cdots$ & 02022, 00 & 02022,02 & $02022,2-2$ & 02022, 20 & $01111,1-1$ & 01111,11 & 00200, 00 & $\ldots$ \\
\hline \multicolumn{10}{|l|}{$\ldots$} \\
\hline 02022,00 & & $E_{v r}$ & $q_{5}$ & $r_{45}$ & $q_{4}$ & $\mathrm{~K}_{3 / 245}$ & $\mathrm{O}_{3 / 245}$ & $x$ & \\
\hline 02022,02 & & & $E_{v r} \pm u_{55}$ & $q_{455} \pm q_{4}$ & $r_{45} \pm u_{45}$ & $\pm \mathrm{O}_{3 / 245}$ & $\mathrm{~K}_{3 / 245}$ & $x$ & \\
\hline $02022,2-2$ & & 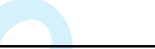 & & $E_{v r} \pm r_{4455}$ & $q_{5} \pm q_{445}$ & $\mathrm{~K}_{3 / 245}$ & $\mathbf{x}$ & $x$ & \\
\hline 02022,20 & & & & & $\mathrm{E}_{\mathrm{vr}} \pm \mathrm{u}_{44}$ & $\mathrm{O}_{3 / 245}$ & $\mathrm{~K}_{3 / 245}$ & $x$ & \\
\hline 01111, 1 -1 & & 8 & & & & $E_{v r} \pm r_{45}$ & $q_{5} \pm q_{4}$ & $\mathrm{~K}_{3 / 245}$ & \\
\hline 01111,11 & & & & & & & $\mathrm{E}_{\mathrm{vr}} \pm \mathrm{u}_{45}$ & $\mathrm{O}_{3 / 245}$ & \\
\hline 00200,00 & & 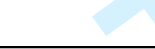 & 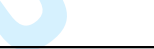 & & & & & $\mathrm{E}_{\mathrm{vr}}$ & \\
\hline$\ldots$ & & & 2 & & & & & & \\
\hline
\end{tabular}


Figure 25 (IN COLOUR): Reduced energy graph, as defined in Eq. (3.5), illustrating the complexity of the vibration-rotation energy pattern in the overtone range in acetylene $\left({ }^{12} \mathrm{C}^{13} \mathrm{CH}_{2}\right)$. The energy behaviour is predicted from the global picture presented in [28]. Full and doted lines indicate $e$ and $f$ parity, respectively. Colours refer to different symmetries, with those corresponding to interacting $k=1$ (blue), 3 (purple) and 5 (black) states highlighted.

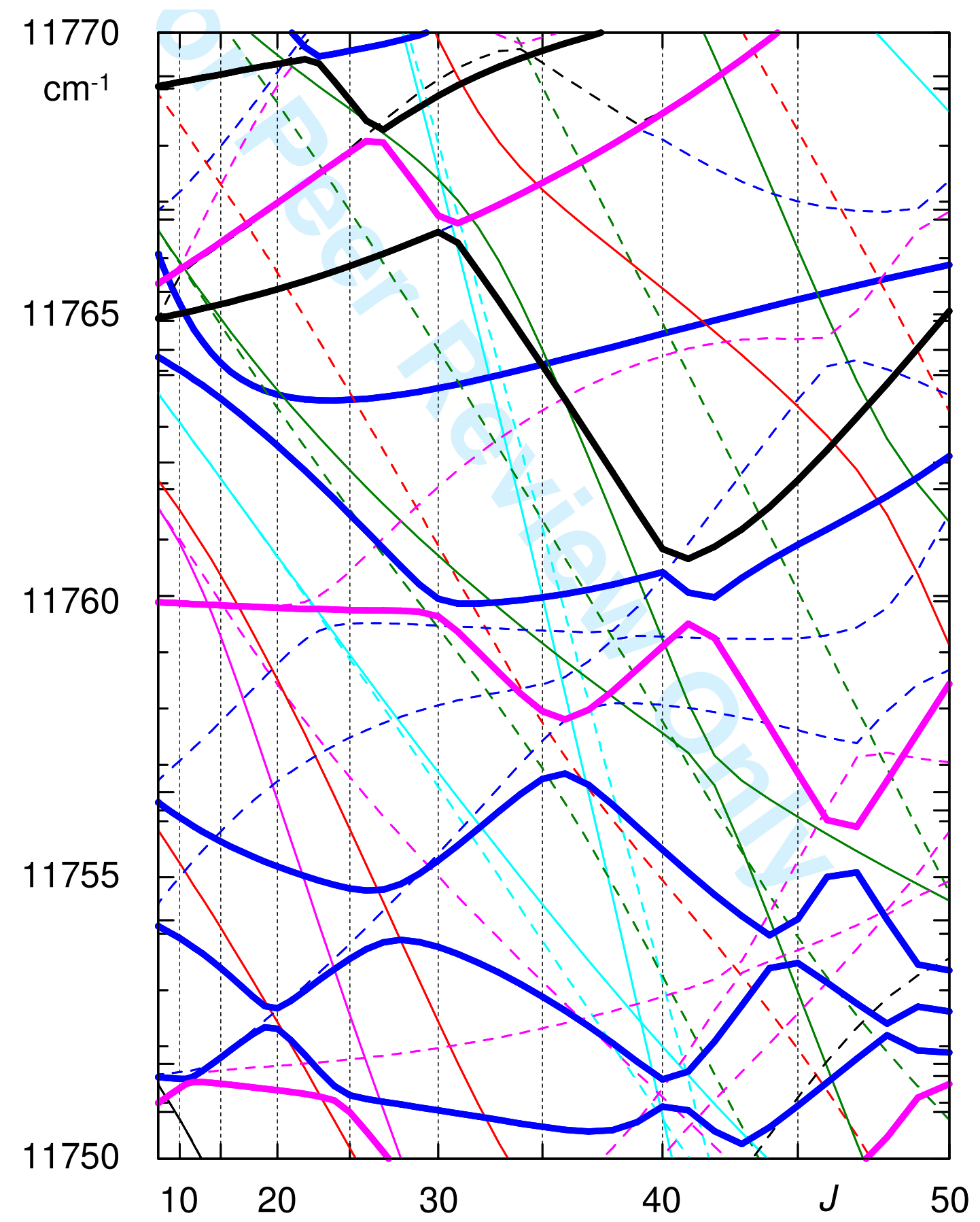


Figure 26: Simulated absorption spectrum of ${ }^{12} \mathrm{C}_{2} \mathrm{H}_{2}$ (line-strengths) around the $v_{5}$ region, corresponding to $500 \mathrm{~K}$ temperature conditions [87]. Population on all excited states up to $v_{4}=5$ was accounted for in the hot band simulation [87].

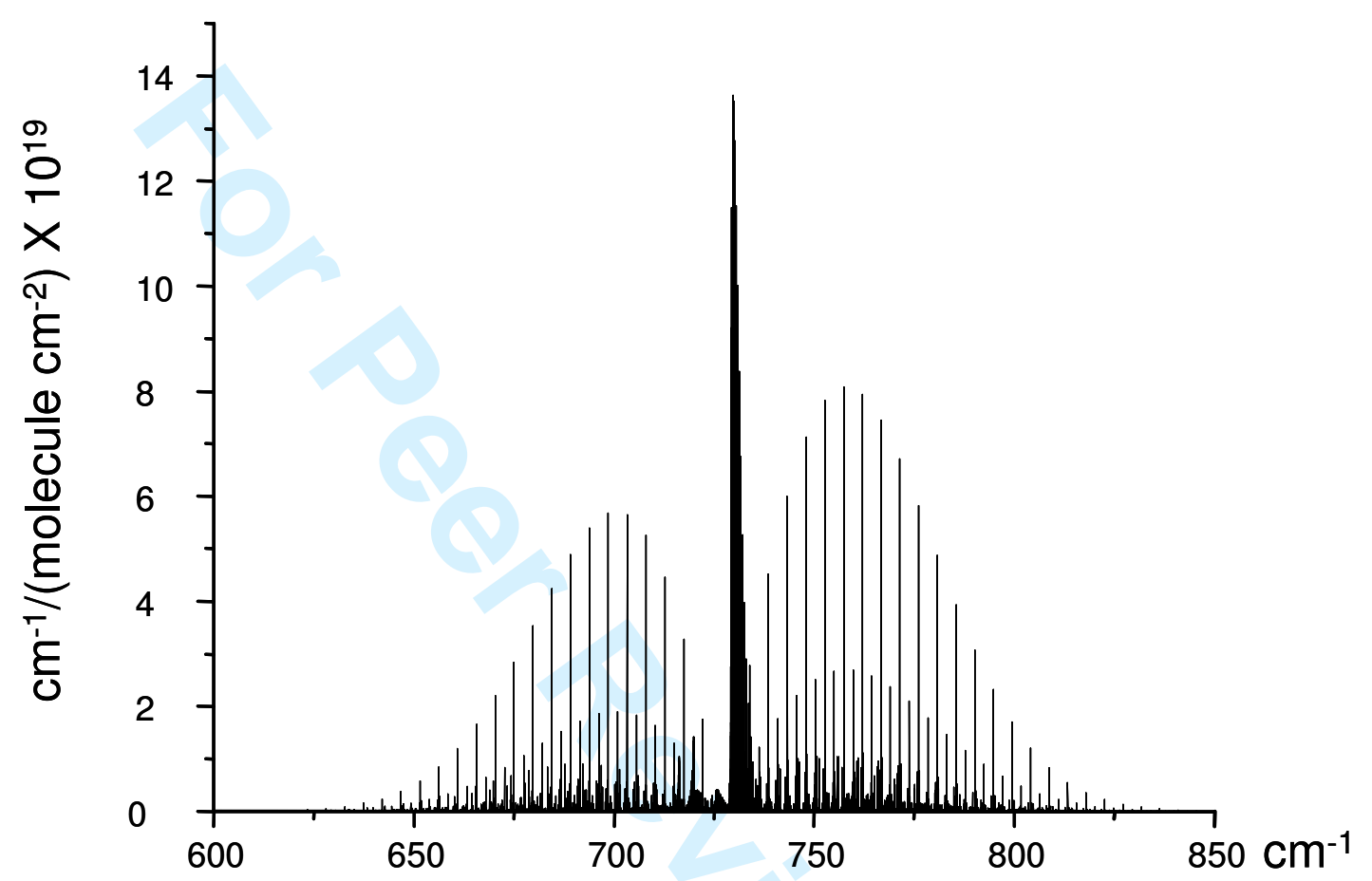


Figure 27: Statistical weights $\left(g_{I}\right)$ as a function of the $e / f$ wavefunction parity of the vibration-rotation energy states in various isotopologues of acetylene. The result depends on the parity of $J$ as well as on the vibrational state-symmetry.

\begin{tabular}{|c|c|c|c|c|c|c|}
\hline & Parity & $J$ & ${ }^{12} \mathrm{C}_{2} \mathrm{H}_{2}$ & ${ }^{12} \mathrm{C}_{2} \mathrm{D}_{2}$ & ${ }^{13} \mathrm{C}_{2} \mathrm{H}_{2}$ & ${ }^{13} C_{2} D_{2}$ \\
\hline$\Sigma_{g}^{+}, \Pi_{g}, \ldots$ & $e$ & even & 1 & 6 & 10 & 15 \\
\hline 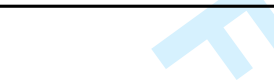 & & odd & 3 & 3 & 6 & 21 \\
\hline \multirow[t]{2}{*}{$\Sigma_{g}^{-}, \Pi_{g}, \ldots$} & $f$ & even & 3 & 3 & 6 & 21 \\
\hline & & odd & 1 & 6 & 10 & 15 \\
\hline \multirow[t]{2}{*}{$\Sigma_{u}^{+}, \Pi_{u}, \ldots$} & e & even & 3 & 3 & 6 & 21 \\
\hline & & odd & 1 & 6 & 10 & 15 \\
\hline \multirow[t]{2}{*}{$\Sigma_{u}^{-}, \Pi_{u}, \ldots$} & $f$ & even & 1 & 6 & 10 & 15 \\
\hline & & odd & 3 & 3 & 6 & 21 \\
\hline
\end{tabular}


Figure 28 Nuclear spin statistics and intensity alternation in $R$ branches of $\Sigma_{(u)}^{+}-\Sigma_{(g)}^{+}$acetylene transitions (see [88]). The parity of $J$ for the stronger lines depends on the total nuclear spin function, while intensity alternation does not occur for asymmetric isotopologues.
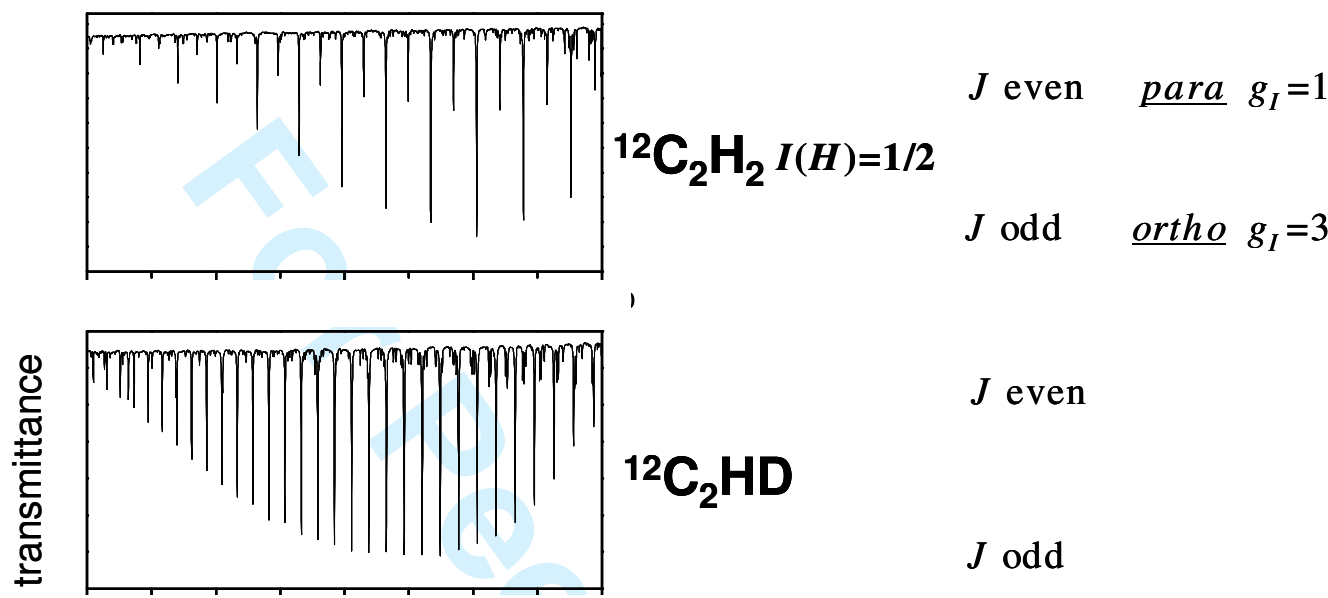

$J$ even

${ }^{12} C_{2} H D$

$J$ odd

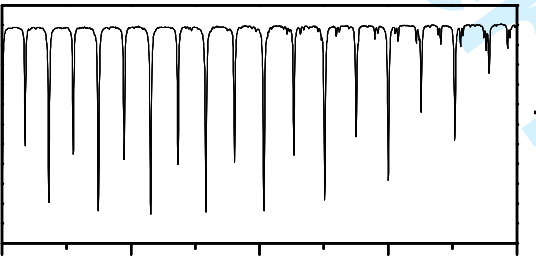

$J$ even ortho $g_{I}=6$

${ }^{12} C_{2} D_{2} I(D)=1$

$J$ odd para $g_{I}=3$

wavenumber 
Figure 29: Evolution of the absorption line profile of the first $\mathrm{R}(J)$ transitions in the $v_{1}+v_{3}$ band of ${ }^{12} \mathrm{C}_{2} \mathrm{H}_{2}$ recorded at ULB with a tunable diode laser in an axisymmetric jet (see experimental details in [95]. The central dip is attributed to the formation of van der Waals species that is more favourable in the central part of the jet beam. The evolution with $J$ may possibly indicate that rotation hinders the formation of these molecular complexes.

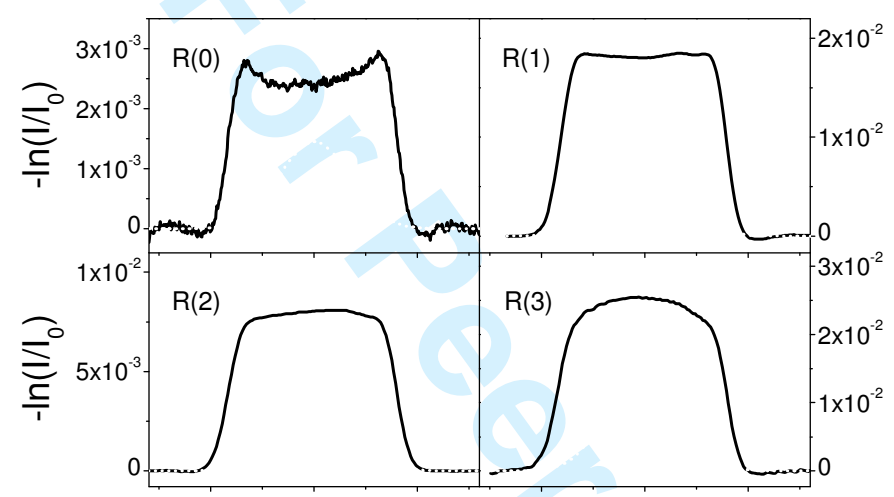


Figure 30: Scheme of the perturbation mechanism possibly explaining unusual self broadening coefficients affecting $J=18$ in $v_{1}+3 v_{3},{ }^{12} \mathrm{C}_{2} \mathrm{H}_{2}$ (see text). ( adapted from [61]).

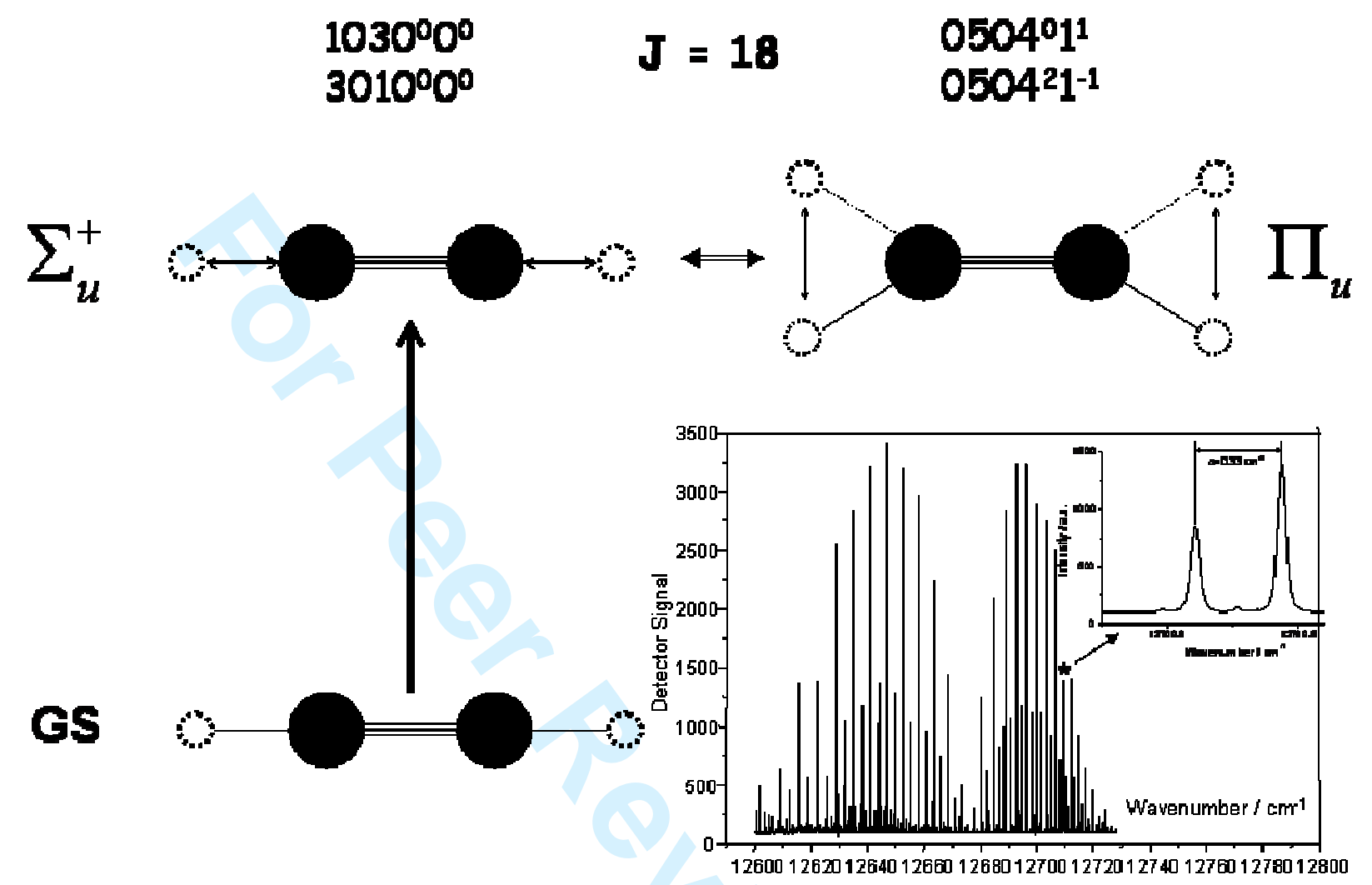


Figure 31: Portion of the absorption spectrum of ${ }^{12} \mathrm{C}_{2} \mathrm{H}_{2}$-Ar recorded using jet-cooled cavity ring down spectroscopy at ULB. Lines in the $K_{\mathrm{a}}=1 \bullet 0$ sub-band of the $2 \mathrm{CH}$ overtone band are assigned in terms of the usual $\mathrm{R} / \mathrm{Q} / \mathrm{P}(J)$ notation, adapted from [102].

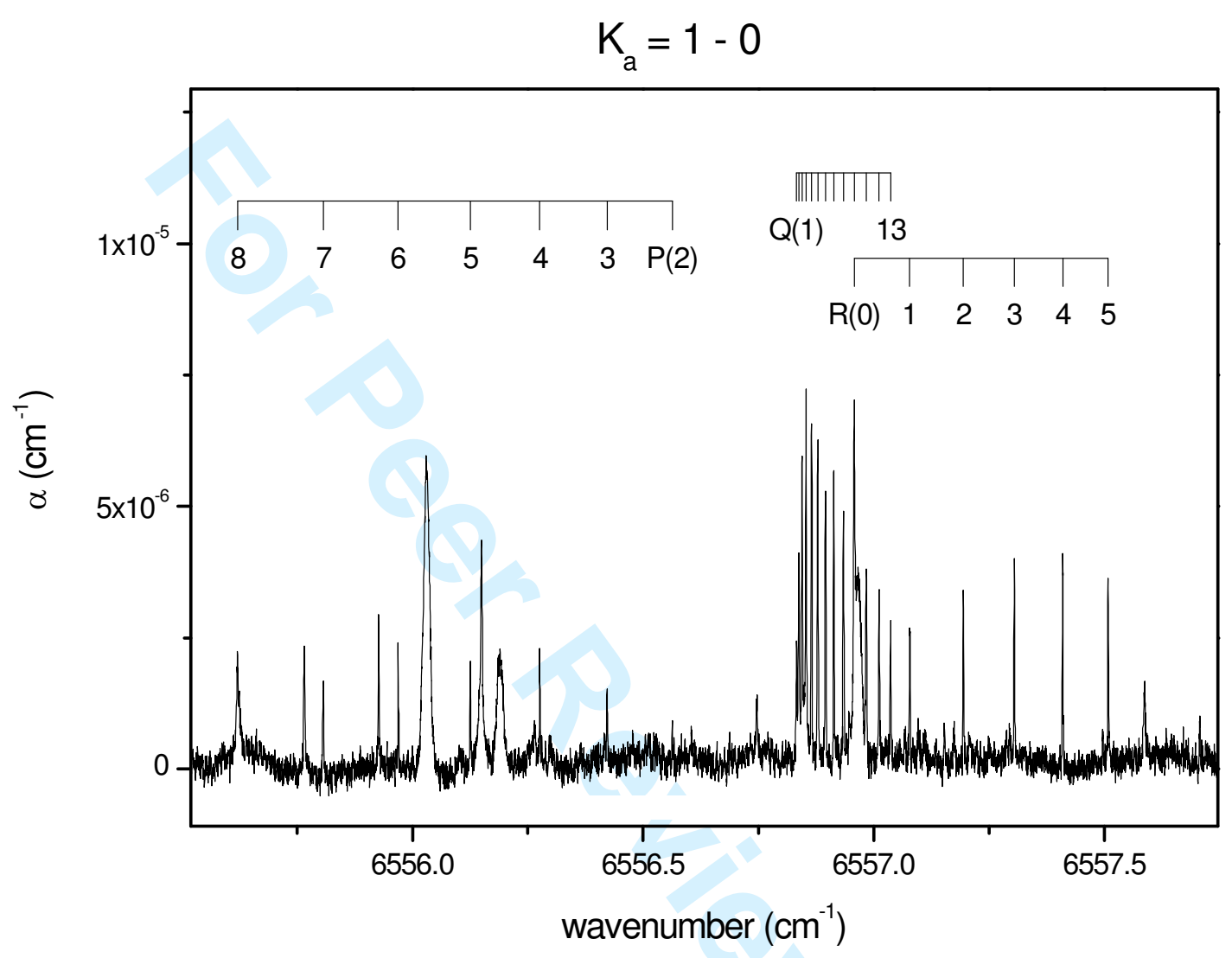


Figure 32: Absorption Fourier transform spectrum of jet-cooled acetylene recorded at ULB demonstrating clustering effects (adapted from [103]).

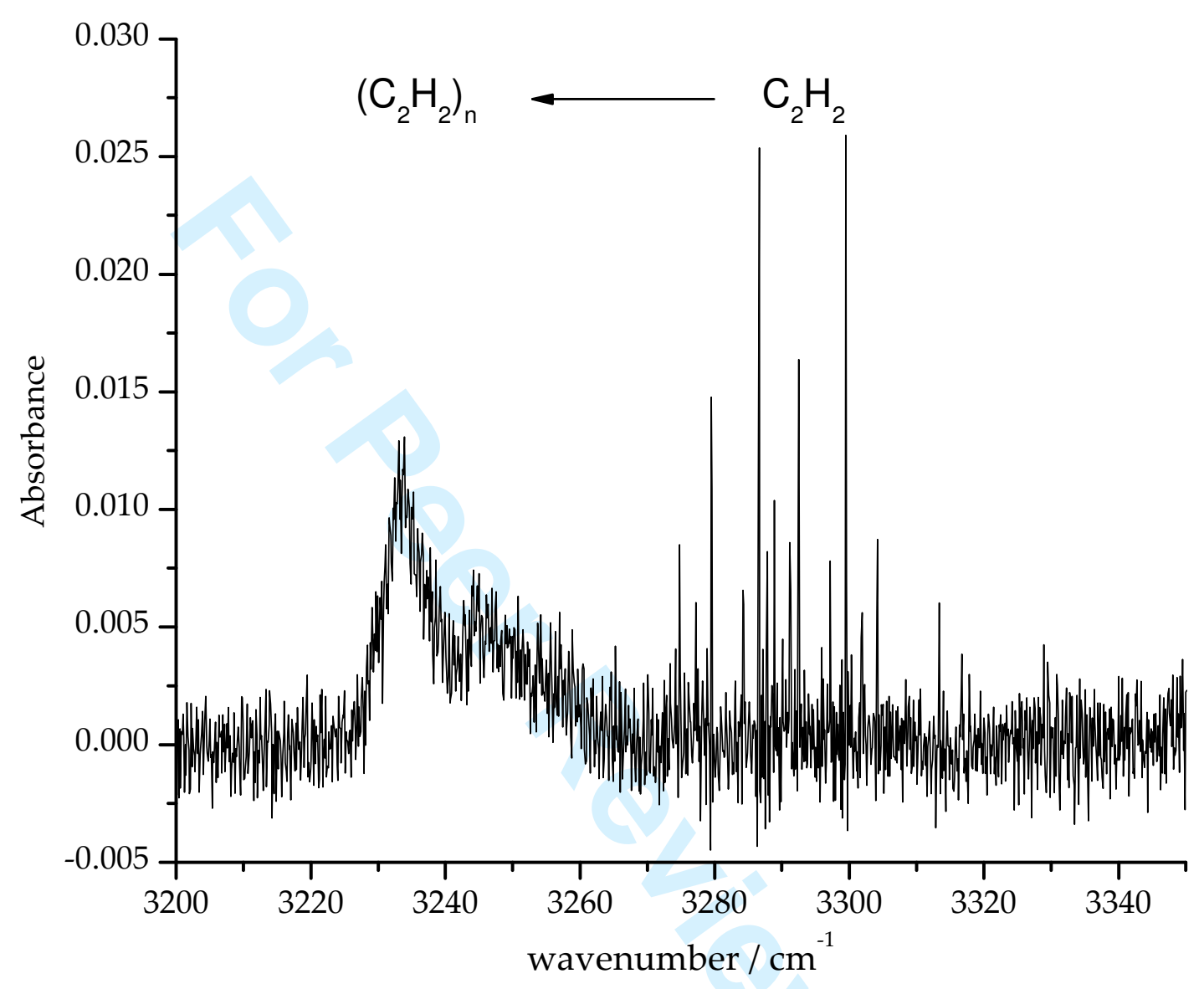


Tables

Table 1: Check of $x$ - $K$ relations in symmetric acetylene isotopologues. (Parameters from El Idrissi et al., 1999 [24] $\left({ }^{12} \mathrm{C}_{2} \mathrm{H}_{2}\right)$; Herman et al., 1998 [25] $\left({ }^{12} \mathrm{C}_{2} \mathrm{D}_{2}\right)$; Di Lonardo et al., $\left.1999[26]\left({ }^{13} \mathrm{C}_{2} \mathrm{H}_{2}\right)\right)$

\begin{tabular}{|c|c|c|c|}
\hline & $12 \mathrm{C}_{2} \mathrm{H}_{2}$ & $\mathrm{I}_{2} \mathrm{C}_{2}$ & ${ }^{13 \mathrm{C}_{2} \mathrm{H}_{2}}$ \\
\hline$x_{11}$ & -24.8 & -12.3 & -25.4 \\
$x_{33}$ & -27.6 & -15.4 & -26.7 \\
\hline$X_{13}$ & -107.5 & -47.5 & -104.3 \\
\hline$K_{1133}$ & -105.7 & -47.2 & -103.4 \\
\hline
\end{tabular}


Table 2: Periods associated to the normal mode vibrational frequencies $\left(\tilde{\omega}_{i}^{e}\right)$ in symmetric acetylene isotopologues as published at the time (from [43]).

\begin{tabular}{|c|c|c|c|c|c|c|}
\hline \multirow{2}{*}{ Mode } & \multicolumn{2}{|c|}{$\mathbf{1 2}_{\mathbf{2}} \mathbf{H}_{\mathbf{2}}$} & \multicolumn{2}{c|}{$\mathbf{1 3}_{\mathbf{2}} \mathbf{H}_{\mathbf{2}}$} & \multicolumn{2}{c|}{$\mathbf{1 2}_{\mathbf{2}} \mathbf{D}_{\mathbf{2}}$} \\
\hline$i$ & $\left(\mathrm{~cm}^{-1}\right)$ & $(\mathrm{fsec})$ & $\left(\mathrm{cm}^{-1}\right)$ & $(\mathrm{fsec})$ & $\left(\mathrm{cm}^{-1}\right)$ & $(\mathrm{fsec})$ \\
\hline 1 & 3506.91 & 9.5 & 3481.14 & 9.6 & 2785.47 & 12.0 \\
\hline 2 & 2012.56 & 16.6 & 1946.93 & 17.1 & 1787.44 & 18.7 \\
\hline 3 & 3421.17 & 9.8 & 3404.99 & 9.8 & 2512.32 & 13.3 \\
\hline 4 & 622.73 & 53.6 & 613.57 & 54.4 & 519.44 & 64.2 \\
\hline 5 & 746.89 & 44.7 & 743.96 & 44.8 & 549.02 & 60.8 \\
\hline
\end{tabular}


Table 3: Principal rotational constants, $\mathrm{B}_{0}$ in $\mathrm{cm}^{-1}$, of various isotopologues of acetylene (from Cané et al., 2002 [69] $\left({ }^{13} \mathrm{C}_{2} \mathrm{D}_{2}\right)$; Di Lonardo et al., unpublished [76] $\left({ }^{13} \mathrm{C}_{2} \mathrm{HD}\right),\left(\mathrm{H}^{12} \mathrm{C}^{13} \mathrm{CD}\right)$, and $\left(\mathrm{H}^{13} \mathrm{C}^{12} \mathrm{CD}\right)$; Di Lonardo et al., 1993 [77] $\left({ }^{13} \mathrm{C}_{2} \mathrm{H}_{2}\right)$; Wlodarczak et al., 1989 [78] $\left({ }^{12} \mathrm{C}_{2} \mathrm{HD}\right)$; Robert et al., 2007 [40] $\left({ }^{12} \mathrm{C}_{2} \mathrm{H}_{2}\right)$; Huet et al., $1991[23]\left({ }^{12} \mathrm{C}_{2} \mathrm{D}_{2}\right) ;$ [79] $\left({ }^{13} \mathrm{C}^{12} \mathrm{CD}_{2}\right)$, and Fayt et al., $\left.2007[28]\left({ }^{12} \mathrm{C}^{13} \mathrm{CH}_{2}\right)\right)$.

$\begin{array}{ll}{ }^{13} \mathrm{C}_{2} \mathrm{D}_{2} & 0.8178722066(4560) \\ { }^{13} \mathrm{C}_{2} \mathrm{HD} & 0.950330801(617) \\ \mathrm{H}^{12} \mathrm{C}^{13} \mathrm{CD} & 0.97527102(68) \\ \mathrm{H}^{13} \mathrm{C}^{12} \mathrm{CD} & 0.96719254(82) \\ { }^{13} \mathrm{C}_{2} \mathrm{H}_{2} & 1.119574687(146) \\ { }^{12} \mathrm{C}_{2} \mathrm{HD} & 0.9915278(3) \\ { }^{12} \mathrm{C}_{2} \mathrm{H}_{2} & 1.17664618(1) \\ { }^{12} \mathrm{C}_{2} \mathrm{D}_{2} & 0.84787420(24) \\ { }^{12} \mathrm{C}^{13} \mathrm{CD} 2 & 0.83305(4) \\ { }^{12} \mathrm{C}^{13} \mathrm{CH}_{2} & 1.14846077(1)\end{array}$

